\title{
Engineering in vivo production of $\alpha$-branched polyesters
}

\author{
Hongjun Dong, Stephanie Liffland, Marc A. Hillmyer, and Michelle C. Y. Chang
}

\section{Materials and Methods}

$\begin{array}{ll}\text { Commercial materials } & \text { S2 }\end{array}$

Bacterial strains $\quad$ S2

Plasmid construction $\quad$ S3

Expression and purification of heterologous expressed enzymes

Preparation of 3-oxo-2-methylbutyryl-CoA and 3R-hydroxy-2-methylbutyryl-CoA substrates S6

$\begin{array}{ll}\text { Enzyme assays } & 57\end{array}$

$\begin{array}{lll}\text { Polyester production } & \text { S8 }\end{array}$

Polyesters extraction from E. coli $\quad$ S9

Polyester quantification analysis $\quad S 9$

Characterization of branched polymer by Nuclear Magnetic Resonance (NMR) S10

Size exclusion chromatography (SEC) of PHAs S11

$\begin{array}{ll}\text { Thermal characterization of PHAs } & \text { S11 }\end{array}$

\section{Supplementary Data}

Table S1. Strains, plasmids, oligonucleotides, and sequences $\quad \mathrm{S} 12$

Figure S1. Comparison of two thiolases AsAcat3 and RePhaA S21

Figure S2. PHA gene cluster in Candidatus accumulibacter phophatis clade IIA str. UW-1 S22

Figure S3. Characterization of PHA polymerases RePhaC and CapPhaEC S23

Figure S4. Synthesis of 3-oxo-2-methylbutyryl-CoA and 3R-hydroxy-2-methylbutyryl-CoA S24

Figure S5. Characterization of the CapPhaBO ketoreductase

Figure S6. CapMaoC1/2 for PHA production

Figure S7. Characterization of RePhaB Q150C S27

Table S2. Confirmation of mutagenesis quality of RePhaB pocket by sequencing S30

Figure S8. High throughput screening of RePhaB library by polyester fermentation S31

Figure S9. Quantification of hydroxyacids monomer composition by LC/QQQ S32

Figure S10. RePhaB pocket library screening S33

Figure S11. Polyester production by the RePhaB Q150 and I186 mutants S34

Table S3. Monomer compositions of PHA polyester samples for polymer characterization S35

Figure S12. NMR spectra of PHA polyesters S36

Figure S13. Chloroform size-exclusion chromatography (SEC) of PHA polyesters S44

Figure S14. THF size-exclusion chromatography (SEC) analysis of PHA polyesters

Figure S15. Thermogravimetric analysis (TGA) of PHA polyesters $S 46$

Figure S16. Differential scanning calorimetry (DSC) analysis of PHA polyesters

Literature Cited $\$ 50$ 


\section{Materials and Methods}

Commercial materials. LB Broth Miller, LB Agar Miller, Terrific Broth (TB), glycerol, Chloramphenicol (Cm) were purchased from EMD Biosciences (Darmstadt, Germany). D-(+)glucose was purchased from MP Biochemicals (Santa Ana, CA). Carbenicillin (Cb), kanamycin $(\mathrm{Km})$, isopropyl- $\beta$-d-thiogalactopyranoside (IPTG), EMD Millipore MultiScreenHTS 96-Well Filter Plates, Dithiothreitol (DTT), HEPES, and Acetonitrile were purchased from Fisher Scientific (Pittsburgh, PA). Bottle top filter 150mL, 24-Well Deep Well Plates, Rectangular were purchase from VWR (Radnor, PA). Thermo Scientific Nunc 96 Well, DeepWell Rnd Btm was purchase from Spectrum Chemicals \& Laboratories Products (Gardena, CA). 5 mm glass beads were purchased from EMD Millipore (Billerica,MA). Aeraseal Sealing Film was purchased from Research Products International (Mount Prospect, IL). Restriction enzymes, T4 DNA ligase, Phusion DNA polymerase, T5 exonuclease, Taq DNA ligase were purchased from New England Biolabs (Ipswich, MA). DNA purification kits, NTA agarose were purchased from Qiagen (Valencia, CA). Oligonucleotides and gBlocks were purchased from Integrated DNA Technologies (Coralville, IA). Deoxynucleotides (dNTPs) were purchased from Invitrogen (Carlsbad, CA). Acrylamide/Bis-acrylamide, SDS, Bio-Rad Protein Assay reagent, ammonium persulfate, N,N,N',N'-tetramethyl-ethane-1,2-diamine (TEMED) were purchase from Bio-Rad Laboratories (Hercules, CA). Imidazole was purchase from Acros Organics (Morris Plains, NJ). cOmplete EDTA-free protease inhibitor was purchased from Roche Applied Science (Penzberg, Germany). Amicon Ultra spin concentrators were purchased from Merck Millipore (Cork, Ireland). Tris-(2-carboxyethyl)phosphine hydrochloride (TCEP) was purchased from Biosynth, Inc. (Itasca, IL). PageRuler ${ }^{\mathrm{TM}}$ Plus prestained protein ladder was purchased from Fermentas (Glen Burnie, Maryland). MOPS, sodium propionate, lysozyme from chicken egg white, $\beta$-Mercaptoethanol (BME), Bovine Serum Albumin (BSA), DL-3Hydroxybutyryl coenzyme A lithium salt, Crotonoyl coenzyme a trilithium salt, Coenzyme A sodium salt hydrate (CoA), Acetoacetyl-CoA, $\beta$-Nicotinamide adenine dinucleotide reduced dipotassium salt (NADH), $\beta$-Nicotinamide adenine dinucleotide 2 '-phosphate reduced tetrasodium salt (NADPH), $\beta$-Nicotinamide adenine dinucleotide hydrate (NAD+), $\beta$ Nicotinamide adenine dinucleotide phosphate disodium salt (NADP+), 5,5'-Dithiobis(2nitrobenzoic acid) (DTNB), Formic acid, Adipic acid, Sodium 3-hydroxybutyrate, Thiophenol, 4-(Dimethylamino)pyridine (DMAP), Ethyl 3-methyl-2-oxobutyrate, N,N'Dicyclohexylcarbodiimide (DCC), Chloroform-d, Poly[(R)-3-hydroxybutyric acid], Poly(3hydroxybutyric acid-co-3-hydroxyvaleric acid, Trichloroacetic acid (TCA) were purchased from Sigma-Aldrich (St. Louis, MO). 2-Methyl-3-hydroxybutyric acid was purchased from Santa Cruz Biotechnology (Dallas, Texas).

Bacterial strains. The $\mathrm{T} 1$ bacteriophage resistant strain E. coli $\mathrm{DH} 10 \mathrm{~B}^{\mathrm{T} 1 \mathrm{R}}$ was used for plasmid construction and BL21(DE3) ${ }^{\mathrm{T} 1 \mathrm{R}}$ was used for heterologous production of proteins for purification. E. coli $\mathrm{BAP} 1^{1}$ or BAP $1^{\mathrm{T} 1 \mathrm{R}}$ was used for propionate-supplemented production of polyester and hydroxy acids. E. coli BAP1 $1^{\text {T1R }}$ was derived from BAP1 by knocking out fhuA 
gene $^{2}$ for additional $\mathrm{T} 1$ bacteriophage resistance. To construct BAP1 ${ }^{\mathrm{T} 1 \mathrm{R}}$, the lambda Red recombination method ${ }^{3}$ was applied. Firstly, the kanamycin resistance gene cassette with homologous tails of $f h u A$ gene was amplified from pKD4 vector using primers 675-fhuA-kan$\mathrm{F}$ and 676-fhuA-kan-R, then transformed into strain BAP1 with ready expression of lambda Red recombinase from vector pKD46. The resulting kanamycin resistant colonies were confirmed to own the insertion of kanamycin cassette into fhuA gene by PCR with primers 677fhuA-test-F and 678-fhuA-test-R. Finally, the kanamycin cassette was recycled by introducing $\mathrm{pCP} 20$, and vectors were cured to get final strain named BAP1 ${ }^{\mathrm{T} 1 \mathrm{R}}$.

Chemically competent cells were prepared using Transformation-Storage Solution (TSS, 10\% $w / v$ PEG 3350, 5\% v/v DMSO, $20 \mathrm{mM} \mathrm{MgCl} 2$ in LB broth). Plasmid transformation was performed by adding $20 \mu \mathrm{L} 5 \times \mathrm{KCM}$ solution $\left(0.5 \mathrm{M} \mathrm{KCl}, 0.15 \mathrm{M} \mathrm{CaCl}_{2}, 0.25 \mathrm{M} \mathrm{MgCl}_{2}\right)$, plasmids and supplementing sterile water to $100 \mu \mathrm{L}$ per $100 \mu \mathrm{L}$ competent cells. After $10 \mathrm{~min}$ incubation on ice, cells were heat-shocked at $42^{\circ} \mathrm{C}$ for $90 \mathrm{~s}$, then diluted to $1 \mathrm{~mL}$ with LB broth, and incubated at $37^{\circ} \mathrm{C}$ for $1 \mathrm{~h}$ before plating on LB agar containing appropriate antibiotics. Electroporation competent cells were prepared using $10 \% v / v$ glycerol solution, and transformed with plasmids by Bio-Rad MicroPulser electroporator.

Plasmid construction. Plasmid construction was carried out using standard molecular biology techniques and the Gibson protocol ${ }^{4}$. PCR amplifications were carried out with Phusion DNA polymerase, following manufacturer instructions. Primer sequences and gene sequences are listed in Table S6 and Table S7. Constructs were verified by sequencing.

Thiolase-Ketoreductase plasmids (Plasmid 1):

pAsAcat3-RePhaB vectors: NphT7 from the NphT7.RePhaB.RrPhaC operon house in pPOL $4.1^{5}$ was replaced with the AsAcat 3 thiolase from Ascaris suum 6 . The acat 3 gene was amplified from pCWori-A3AsH1 $1^{6}$ using the 112-pCWori-A3AsH11 FWD/113-pCWoriA3AsH11 REV primer pair. The vector backbone was amplified from pPOL4.1 using the 110pPOL4.1 FWD/111-pPOL4.1 REV primer pair, eliminating the $n p h T 7$ gene. Gibson assembly yielded pPOL4.1-AsAcat3, which was digested with Sall to remove $R r P h a C$ gene and selfligated, yielding pAsAcat3-RePhaB.

Other pAsAcat3-ketoreductase vectors: pPOL4.1-AsAcat3 was digested with EcoRI and SpeI, CapPhaB0-3 were amplified from the corresponding synthetic gBlocks using primer pairs 142-RBS-CapPhaB0 FWD/143-RBS-CapPhaB0 REV, 144-RBS-CapPhaB1 FWD/145-RBSCapPhaB1 REV, 146-RBS-CapPhaB2 FWD/147-RBS-CapPhaB2 REV, 148-RBSCapPhaB3 FWD/149-RBS-CapPhaB3 REV, respectively. The resulting PCR products were assembled with the vector backbone to generate pAsAcat3-CapPhaB0-RrPhaC, pAsAcat3CapPhaB1-RrPhaC, pAsAcat2-CapPhaB2-RrPhaC, pAsAcat3-CapPhaB3-RrPhaC. These vectors were digested with SalI to remove $R r P h a C$ gene and self-ligated to yield pAsAcat3CapPhaB0, pAsAcat3-CapPhaB1, pAsAcat2-CapPhaB2, pAsAcat3-CapPhaB3, respectively. AsHadh2 was amplified from pCWori-A3AsH11 ${ }^{6}$ using the 190-pCWori-A3AsH11 
FWD/191-pCWori-A3AsH11 REV primer pair and treated as described for the CapPhaB constructs to generate pAsAcat3-AsHadh2. CaHbd was amplified from pT5T33-Bu2 ${ }^{7}$ using the 763-499hbd-F/764-499hbd-R primer and inserted into pAsAcat3-AsHadh2, generating pAsAcat3-CaHbd. The CapPhaB0.PhaA operon was amplified from the corresponding gBlock using the primers 152-RBS-phaBA FWD/153-RBS-phaBA REV primer pair. The vector backbone was then amplified from pPOL4.1-AsAcat3 using the 150-pPOL4.1AsAcat3 FWD/151-pPOL4.1-AsAcat3 RE primer pair and assembled with CapPhaB0.PhaA, to generate $\mathrm{pCapPhaB0.PhaA.RrPhaC}$. This vector was digested by SalI to remove $R r P h a C$, self-ligated to generate $\mathrm{pCapPhaB0.PhaA.}$

RePhaA vectors: RePhaA was amplified from pT5T33-phaA.HBD-crt ${ }^{7}$ using the 713pT5T33-phaA.HBD-crt FWD/714-pT5T33-phaA.HBD-crt REV primer pair. The vector backbone was amplified from pA3Acat3-RePhaB using the 711-pA3B FWD/712-pA3B REV primer pair. These two fragments were assembled to generate pRePhaA.RePhaB. For pRePhaA-AsHadh2, RePhaA was amplified from pT5T33-phaA.HBD-crt using the 717pT5T33-phaA.HBD-crt FWD/718-pT5T33-phaA.HBD-crt REV primer pair. The vector backbone was amplified from pAsAcat3-AsHadh2 using the 715-pA3H2 FWD/716-pA3H2 REV primer pair and assembled with RePhaA.

PHA polymerase plasmids (Plasmid 2):

pT7-CapPhaCl and pT7-CapPhaEC: These vectors were assembled by amplification of the $\mathrm{CapPhaC} 1$ and CapPhaEC genes from their gBlocks using the CAPphaC1 FWD/CAPphaC1 REV and CAPphaEC3 FWD/CAPphaEC3 REV primer pairs, respectively, and insertion into a vector backbone derived from pT7-TE3. ${ }^{6}$ These vector backbones were obtained by amplification of pT7-TE3 using the 114-pT7-TE3 FWD/115-pT7-TE3 REV and 116-pT7TE3 FWD/117-pT7-TE3 REV pairs for pT7-CapPhaC1 and pT7-CapPhaEC, respectively.

pT7-AcPhaPC*: The backbone was amplified from pT7-TE3 using the 154-pT7-TE3 FWD/155-pT7-TE3 REV1 primer pairs and ligated with the AcPhaPC*J gBlock to generate pT7-AcPhaPC*J. Amplification of this vector using the 188-pT7-phaPC-F/189-pT7-phaPC$\mathrm{R}$ primer pair allowed the removal of AcPhaJ after self-ligation to generate $\mathrm{pT} 7-\mathrm{AcPhaPC}$ *. pT7-RePhaC: RePhaC was amplified from pPOL1 ${ }^{5}$ using the 290-pPOL1 FWD/291-pPOL1 REV primer pair. The vector backbone was amplified from pT7-CapPhaC1 using the 288pT7-CAPphaC1 FWD/289-pT7-CAPphaC1 REV primer pairs. These two fragments were assembled.

pT7-CapPhaEC-CpPct: CpPct was amplified from pT7-TE3/Pct ${ }^{6}$ using the 82-Pct-1/84-Pct3 primer pair and inserted into pT7-RePhaC was digested by PstI and SalI.

pRSF-CapMaoC1/2 (Plasmid 3):

$\mathrm{CapMaoC} 1 / 2$ genes were amplified from the synthetic gBlock using the CAPmaoC1-maoC2 FWD/CAPmaoC1-maoC2 REV primer pair and inserted into pRSFDuet-1 amplified using the 122-pRSFDuet-1 FWD and 123-pRSFDuet-1 REV primer pair. 


\section{Expression vectors:}

pET16b-CapPhaB0: CapPhaB0 was amplified with the 186-CapPhaB0 FWD/187-CapPhaB0 REV primer pair and inserted into $\mathrm{pET} 16 \mathrm{~b}$ digested with NdeI and BamHI.

pET16b-RePhaB and pET16b-RePhaB_Q150C: These plasmids were constructed by assembly of RePhaB or RePhaB_Q150C amplified using the 679-pA3B FWD\&680-pA3B REV primer pair from pAsAcat3-RePhaB or pAsAcat3-RePhaB_Q150C, respectively, with pET16b digested with NdeI and BamHI.

pET28a-CapPhaEC and pET28a-CapPhaE: CapPhaEC and CapPhaE were amplified from pT7-CapPhaEC using the 725-CAPphaEC FWD/726-CAPphaEC REV and 721-CapPhaE FWD/722-CapPhaE REV primer pairs, respectively, and inserted into the pET28a digested with NcoI and XhoI.

Expression and purification of heterologously expressed enzymes. Table S1 lists amino acid sequences of purified proteins from expression vectors constructed in this study. Other proteins His $_{10}$-PhaJ and Strep-Crt, were purified by Dr. Jeffrey Hanson. ${ }^{6}$

TB $(1 \mathrm{~L})$ containing carbenicillin $(50 \mu \mathrm{g} / \mathrm{mL})$ or kanamycin $(50 \mu \mathrm{g} / \mathrm{mL})$ in a $2.5 \mathrm{~L}$ Ultra yield baffled shake flasks (Thomson Instrument Company, Oceanside, CA) was inoculated to OD $_{600}$ $=0.05$ with an overnight $\mathrm{TB}$ culture of freshly transformed $E$. coli $\mathrm{BL} 21(\mathrm{DE} 3)^{\mathrm{T} 1 \mathrm{R}}$ containing the proper overexpression plasmid. The cultures were grown at $37^{\circ} \mathrm{C}$ at $200 \mathrm{rpm}$ to $\mathrm{OD}_{600}=0.6$ to 0.8 at which point cultures were cooled on ice for $20 \mathrm{~min}$, followed by induction of protein expression with IPTG $(0.4 \mathrm{mM})$ and overnight growth at $16^{\circ} \mathrm{C}$. Cell pellets were harvested by centrifugation at $8,000 \times g$ for $5 \mathrm{~min}$ at $4^{\circ} \mathrm{C}$ and stored at $-80^{\circ} \mathrm{C}$ for protein purification.

Frozen cell pellets were thawed and resuspended at $3 \mathrm{~mL}$ per gram cell paste with Wash Buffer (50 mM HEPES, $10 \mathrm{mM}$ imidazole, $300 \mathrm{mM} \mathrm{NaCl}, 5 \%$ glycerol, $\mathrm{pH}$ 7.5) supplemented with 8 $\mathrm{mM}$ 2-mercaptoethanol, $1 \mathrm{mg} / \mathrm{mL}$ lysozyme, $0.04 \mathrm{uL} / \mathrm{ml}$ benzonase, and one cOmplete EDTAfree protease inhibitor Cocktail tablet per $25 \mathrm{~mL}$. The cells were lysed by sonication using a Misonix Sonicator 3000 , and lysate was centrifuged at $10,000 \times \mathrm{g}$ for $30 \mathrm{~min}$ at $4{ }^{\circ} \mathrm{C}$ to separate soluble and insoluble fractions. The soluble lysate was loaded onto $1.5 \mathrm{~mL} \mathrm{30 \%} \mathrm{Ni-NTA} \mathrm{resin}$ column (Qiagen) by gravity flow. The column was washed with $40 \mathrm{~mL}$ Wash Buffer with $5 \mathrm{mM}$ 2-mercaptoethanol, and $50 \mathrm{~mL}$ mixed buffer of 90\% Wash Buffer with $5 \mathrm{mM} \mathrm{2-}$ mercaptoethanol and 10\% Elution buffer (50 mM HEPES, $250 \mathrm{mM}$ imidazole, $300 \mathrm{mM} \mathrm{NaCl}$, $5 \%$ glycerol, $\mathrm{pH} 7.5$ ) with $5 \mathrm{mM} 2$-mercaptoethanol. Protein was eluted by $10 \mathrm{~mL}$ Elution Buffer with $5 \mathrm{mM}$ 2-mercaptoethanol. Fractions containing the target protein were pooled by A280 nm and concentrated using an Amicon Ultra spin concentrator (Millipore). Finally, protein was then exchanged into Storage Buffer (20 mM HEPES, $100 \mathrm{mM} \mathrm{NaCl}, 10 \%$ glycerol) with $0.2 \mathrm{mM}$ TCEP using an Amicon Ultra spin concentrator. The $\mathrm{pH}$ of Storage Buffer used for RePhaB, RePhaB_Q150C, and CapPhaEC was 9.03, the pH of Storage Buffer used for $\mathrm{CapPhaB} 0$ and $\mathrm{RePhaC}$ was 7.5, and the $\mathrm{pH}$ of Storage Buffer used for PhaE was 5.93. Protein 
concentration was estimated using the Bio-Rad Bradford Protein Assay Kit with BSA as standard. Aliquots were frozen in liquid nitrogen and stored at $-80{ }^{\circ} \mathrm{C}$.

Preparation of 3-oxo-2-methylbutyryl-CoA and 3R-hydroxy-2-methylbutyryl-CoA substrates. The preparation method was slightly modified from previous work. ${ }^{6}$ Free 3 -oxo2-methylbutyric acid were prepared by hydrolyzing $1.5 \mathrm{mmol}$ of the corresponding Ethyl 3methyl-2-oxobutyrate in 1.2 eq of $1 \mathrm{M}$ aqueous $\mathrm{NaOH}$ overnight at room temperature. The reaction was neutralized with $6 \mathrm{M} \mathrm{HCl}$ to $\mathrm{pH} 7$ and extracted three times with ethyl acetate. The aqueous layer was acidified to $\mathrm{pH} 1$ and extracted three times with ethyl acetate. The organic layers were then combined, dried with $\mathrm{Na}_{2} \mathrm{SO}_{4}$, and the solvent removed by rotary evaporation. 3-oxo-2-methylbutyric acid was observed as a light yellow oil. The free acids obtained were then immediately used in the subsequent coupling reaction.

Crude 3-oxoacid products (estimated to be $5 \mathrm{mmol}$ ) from above were dissolved in $30 \mathrm{~mL}$ ethyl acetate in a round bottom flask with a stir bar, to which 2 to $3 \mathrm{mg}$ DMAP and 0.8 eq of thiophenol $(122 \mu \mathrm{L}, 4 \mathrm{mmol})$ were added. The reaction was cooled in an ice bath before dropwise addition of 1 eq of N, N'-Dicyclohexylcarbodiimide (DCC) (5 mmol). Formation of a white precipitant was observed after 5-10 min. The reaction was allowed to continue for $2 \mathrm{~h}$ at $0{ }^{\circ} \mathrm{C}$ and a subsequent $2 \mathrm{~h}$ at room temperature. The reaction mixture was then filtered and the resulting filtrate was extracted three times with $1 \mathrm{M} \mathrm{NaHCO}_{3}$. The organic layer was dried with $\mathrm{Na}_{2} \mathrm{SO}_{4}$ and solvent removed by rotary evaporation.

The crude mixture was dissolved in $100 \mu \mathrm{L}$ acetonitrile and transferred to a stirring solution of 0.4 eq of $\mathrm{CoA}(0.4 \mathrm{mmol})$ in $3 \mathrm{~mL} 0.5 \mathrm{M} \mathrm{NaHCO}_{3}$ in an ice bath. After $1 \mathrm{~h}$ at $0{ }^{\circ} \mathrm{C}$, the reaction was warmed to room temperature and allowed to continue for four hours before quenching by acidification to $\mathrm{pH} 3$ through the addition of formic acid (50\% formic acid, 50\% water). The reaction was then extracted twice with diethyl ether, and nitrogen was passed over the aqueous layer to remove residual ether. Compounds were purified on an Agilent 1200 HPLC system using a Zorbax Eclipse XDB C-18 column ( $5 \mu \mathrm{m}, 9.4 \times 250 \mathrm{~mm}$, Agilent $)$ with a linear gradient over $24 \mathrm{~min}$, starting at $4 \%$ acetonitrile and ending between $50 \%$ acetonitrile, with $100 \mathrm{mM}$ ammonium acetate, $\mathrm{pH} 5.0$, as the aqueous mobile phase $(3 \mathrm{~mL} / \mathrm{min})$. Fractions were screened by ESI-MS and those containing pure 3-oxo-2-methybutyryl-CoA were lyophilized overnight, dissolved in water $\left(1 \mathrm{~mL}\right.$ ), and quantified (extinction coefficient of $\mathrm{A} 260=17800 \mathrm{M}^{-1} \mathrm{~cm}^{-1}$ ), and aliquots were stored at $-80^{\circ} \mathrm{C}$.

To produce the reduced substrates, 5-10 mM 3-oxo-2-methybutyryl-CoA was reduced by 0.1 $\mathrm{mM}$ RePhaB using 1.2 eq of NADPH in HEPES buffer (10 mM HEPES, $150 \mathrm{mM} \mathrm{KCl,} 10 \mathrm{mM}$ $\mathrm{KH}_{2} \mathrm{PO}_{4}, \mathrm{pH} 7.5$ ) for $30 \mathrm{~min}$ at room temperature, to generate $3 R$-hydroxy-2-methylbutyrylCoA. The reaction was monitored by LC/MS. After completion of reaction, protein was removed by Amicon ultra centrifugal filter (EMD Millipore). 
Preparative HPLC fractions and RePhaB reduced $3 R$-hydroxyacyl-CoA products were screened on an Agilent 1290 HPLC system using a Poroshell C18 column (HPH-C18 2.7um, 2.1X100 $\mathrm{mm}$ ) with a linear gradient from 0 to $65 \%$ acetonitrile over $2 \mathrm{~min}$ with $10 \mathrm{mM}$ ammonium formate as the aqueous mobile phase $(0.75 \mathrm{~mL} / \mathrm{min})$. Mass spectra were recorded on an Agilent triple quadrupole MS with ESI source, operating in positive ion scan mode.

CapPhaB0 stereoselectivity assay. This method was modified from a previous study. ${ }^{6}$ For determination of stereoselectivity, CapPhaB0 activity was measured in the oxidative direction by monitoring the formation of NADH at $340 \mathrm{~nm}$. NADH content was quantified using an extinction coefficient of $6.22 \mathrm{mM}^{-1} \mathrm{~cm}^{-1}$ at $340 \mathrm{~nm}$. $3 S$-hydroxybutyryl-CoA or $3 R$ hydroxybutyryl-CoA was formed in situ from crotonyl-CoA and either crotonase (Crt) or PhaJ enzyme. Assays were performed at room temperature in a quartz cuvette in a total volume of $100 \mu \mathrm{L}$ (path length $1 \mathrm{~cm}$ ) containing HEPES buffer (10 mM HEPES, $150 \mathrm{mM} \mathrm{KCl}, 10 \mathrm{mM}$ $\left.\mathrm{KH}_{2} \mathrm{PO}_{4}, \mathrm{pH} 7.5\right), \mathrm{NAD}^{+}$or $\mathrm{NADP}^{+}(0.4 \mathrm{mM})$, varying amounts of crotonyl-CoA, 1 unit of either Crt or PhaJ, and CapPhaB0 (4.79 nM with Crt or $958.71 \mathrm{nM}$ with PhaJ). The reaction was initiated by addition of crotonyl-CoA and mixing rapidly by pipette. $k_{c a t}$ and $K_{M}$ were determined by fitting the initial rate data using Origin (OriginLab, Northampton, MA) using the Michaelis-Menten equation:

$$
v_{0}=v_{\max } *[\mathrm{~S}] /\left(K_{\mathrm{M}}+[\mathrm{S}]\right)
$$

RePhaB and RePhaB_Q150C assays. RePhaB and RePhaB_Q150C activity was measured by monitoring the initial consumption of NADPH at $340 \mathrm{~nm}$ on a Beckman DU 800 spectrophotometer (Beckman, USA). Assays were performed at room temperature in a $1 \mathrm{~cm}$ cuvette in a total volume of $100 \mu \mathrm{L}$ in HEPES buffer (10 mM HEPES, $150 \mathrm{mM} \mathrm{KCl}, 10 \mathrm{mM}$ $\left.\mathrm{KH}_{2} \mathrm{PO}_{4}, \mathrm{pH} 7.5\right)$ with NADPH $(0.15 \mathrm{mM})$, varying amounts of 3-oxoacyl-CoA compound, and RePhaB and RePhaB_Q150C enzyme. The reaction was initiated by addition of 3-oxoacyl-CoA compound and mixing rapidly by pipette. The concentrations of RePhaB for 3-oxo-butyryl-CoA and 3-oxo-2-methylbutyryl-CoA were $17.3 \mathrm{nM}$ and $86.5 \mathrm{nM}$, respectively. The concentrations of RePhaB_Q150C for 3-oxo-butyryl-CoA and 3-oxo-2-methylbutyryl-CoA were $692.8 \mathrm{nM}$ and $1732 \mathrm{nM}$, respectively. The kinetic data for the Q150C mutant fit poorly to the MichaelisMenten, Hill, and substrate inhibition equations. Therefore, we developed a Hill-substrate inhibition equation considering potential cooperativity among subunits with significant substrate inhibition based on previous publications ${ }^{8-9}$ (for the derivation please see Figure S7):

$$
v_{0}=\frac{V_{\max }[B]_{0}^{n}}{K_{M}^{n}+[B]_{0}^{n}\left(1+\frac{[B]_{0}^{n}}{K_{I}^{n}}\right)}
$$

PHA polymerase assay. For RePhaC, the discontinuous DTNB method was used according to previous sutdies ${ }^{10-12}$ with modification. The reaction mixture contained a final volume of 300 
$\mu \mathrm{L}: 100 \mathrm{mM} \mathrm{KPi}$ (pH7.57), $0.2 \mathrm{mg} / \mathrm{mL}$ BSA, $0.05 \mathrm{mg} / \mathrm{mL}$ Triton X-100, and varying amounts of hydroxyacyl-CoA. The reaction was initiated by addition of $\operatorname{RePhaC}(1 \mu \mathrm{M})$ and mixing rapidly by pipette. It was incubated at room temperature, and aliquots $(20 \mu \mathrm{L})$ were removed at timed intervals and quenched by addition of $100 \mu \mathrm{L}$ of ice-cold $5 \%$ trichloroacetic acid. This mixture was centrifuged at $4000 \mathrm{rpm}$ for $30 \mathrm{~min}$ to pelletize the protein. Then, $100 \mu \mathrm{L}$ of supernatant was transferred to $100 \mu \mathrm{L}$ of $0.5 \mathrm{M}$ HEPES ( $\mathrm{pH} 7.5)$ with $3 \mathrm{mM}$ DTNB. The absorbance at $412 \mathrm{~nm}$ (extinction coefficient $=14150 \mathrm{M}^{-1} \mathrm{~cm}^{-1}$ ) was measured after incubation of this mixture for $5 \mathrm{~min}$ at room temperature using a microplate reader (Biotek synergy 2 multimode detection microplate reader). Commercial $D L-3$-hydroxybutyryl-CoA (Sigma-Aldrich (St. Louis, MO) or 3R-hydroxybutyryl-CoA reduced from commercial acetoacetyl-CoA (SigmaAldrich (St. Louis, MO) by RePhaB were used as the substrate. RePhaC did not show detectable activity toward $3 R$-hydroxy-2-methylbutyryl-CoA $(0.5 \mathrm{mM})$ with $1-10 \mu \mathrm{M}$ RePhaC in vitro, while it did show activity toward native substrate $3 R$-hydroxy-butyryl-CoA.

For CapPhaEC, the continuous DTNB method was used because CapPhaEC's activity was not affected in the presence of DTNB (Data not shown). The reaction mixture contained a final volume of $100 \mu \mathrm{L}$ : $100 \mathrm{mM} \mathrm{KPi} \mathrm{(pH} \mathrm{7.57),} 0.2 \mathrm{mg} / \mathrm{mL}$ BSA, $0.05 \mathrm{mg} / \mathrm{mL}$ Triton X-100, $1 \mathrm{mM}$ DTNB, and varying amounts of hydroxyacyl-CoA. The reaction was initiated by addition of CapPhaEC (15.46 nM for 3R-hydroxybutyryl-CoA or $618.4 \mathrm{nM}$ for 3R-hydroxy-2methylbutyryl-CoA) and mixing rapidly by pipette. The absorbance at $412 \mathrm{~nm}$ was monitored by a Beckman DU 800 spectrophotometer (Beckman, USA). The Hill inhibition equation shown below provided a better fit for the kinetic data than the Michaelis-Menten equation.

$$
v_{0}=\frac{V_{\max }[S]_{0}^{n}}{K_{M}^{n}+[S]_{0}^{n}}
$$

In vivo polyester production. Electrocompetent cells of the E. coli BAP1 or BAP $1^{\mathrm{T} 1 \mathrm{R}}$ strain were transformed with the appropriate plasmids (two plasmids bearing the genes for biosynthetic pathway of polymer). For both the polymer production experiment and $\mathrm{PhaB}$ pocket library screening (Figure S11), a fresh, single colony of transformants was used to inoculate $3 \mathrm{~mL}$ of TB broth containing $2 \%(\mathrm{w} / \mathrm{v})$ glucose, $0.2 \%(\mathrm{w} / \mathrm{v})$ sodium propionate, $0.418 \%$ (w/v) MOPS, $50 \mu \mathrm{g} / \mathrm{mL}$ carbenicillin, $50 \mu \mathrm{g} / \mathrm{mL}$ chloramphenicol, and $0.2 \mathrm{mM}$ IPTG in one well of 24 deep rectangular well plates (PDW10ML24CS, Axygen Scientific, Inc., Union City, CA). The 24-well plates were covered by gas permeable AeraSeal sterile film (Research Products International Corp. Mount Prospect, IL), and incubated in a rotary shaker (Innova 44 incubator shaker series, New Brunswick Scientific Company, Inc), at $30^{\circ} \mathrm{C}$ and $200 \mathrm{rpm}$, for 2 days. For polymer production in flasks, fresh transformant colonies from agar plate were inoculated in $10 \mathrm{~mL}$ of TB broth containing $2 \%(\mathrm{w} / \mathrm{v})$ glucose, $0.2 \%(\mathrm{w} / \mathrm{v})$ sodium propionate, $0.418 \%$ (w/v) MOPS, $50 \mu \mathrm{g} / \mathrm{mL}$ carbenicillin, and $50 \mu \mathrm{g} / \mathrm{mL}$ chloramphenicol media, in a tube for overnight culture, which was then diluted to $30 \mathrm{~mL}$ of the same media in $250 \mathrm{~mL}$ baffled culture flasks (Kimble Glass; Chicago, IL) to OD600 $=0.05$. Cultures were grown at $37{ }^{\circ} \mathrm{C}$ and 
$200 \mathrm{rpm}$ for about 3 hours in a rotary shaker to OD600 $=0.4-0.6$, at which point IPTG was added to concentration of $0.2 \mathrm{mM}$. At this time the growth temperature was reduced to $30{ }^{\circ} \mathrm{C}$, and maintained for two days for polymer production.

Polyester extraction from E. coli. Polyesters were isolated using a previously published chloroform extraction method ${ }^{13}$ with modifications. In a standard extraction, cell pellets were first washed twice with distilled water and once with pure ethanol at room temperature. They were then dehydrated overnight at $60{ }^{\circ} \mathrm{C}$ and ground into a powder. Intracellular polyester extraction was performed by stirring the dry cell powder in chloroform $(20 \mathrm{~mL} / \mathrm{g}$ dry cell powder) at $55{ }^{\circ} \mathrm{C}$ overnight. The resulting chloroform mixture was then filtered through Whatman cellulose filter paper to remove any insoluble particles. The clear chloroform solution was condensed by rotary evaporation, and the polyester precipitated by addition of a 10-fold volume of cold ethanol. The solution was then centrifuged, and the resulting polyester pellet dried in air to provide the final dry polymer.

Polyester quantification analysis. For cell samples, cells in $1 \mathrm{~mL}$ culture after 2 days of growth were harvested by centrifugation, and then $200 \mu \mathrm{L}$ of $2 \mathrm{M} \mathrm{KOH}$ was added to the cell pellet. The resulting mixture was incubated in an $80{ }^{\circ} \mathrm{C}$ water bath for 1 hour to allow for cell and polymer alkaline hydrolysis to generate free hydroxy acids. $95 \mu \mathrm{L}$ of mixed acids $(70 \% 6$ $\mathrm{M} \mathrm{HCl}$ and 30\% 6.1 M trichloroacetic acid (TCA)) was added to neutralize the $\mathrm{KOH}$ and precipitate proteins. The mixture was centrifuged at $18000 \mathrm{~g}$ for 10 minutes at room temperature. $10 \mu \mathrm{L}$ of the resulting supernatant was then diluted with $190 \mu \mathrm{L}$ of an internal standard solution of $100 \mu \mathrm{M}$ adipic acid. Samples were then filtered through a 96-well MultiScreen ${ }^{\text {HTS }}$ plate for HPLC/MS analysis.

For extracted pure polyester, 5-10 mg of sample was added to $970 \mu \mathrm{L}$ methanol and $30 \mu \mathrm{L}$ concentrated $\mathrm{H}_{2} \mathrm{SO}_{4}$ in a caped glass scintillation vial. The samples were then incubated for 3 hours at $100{ }^{\circ} \mathrm{C}$ to allow for methyl esterification to occur. $3 \mathrm{~mL} 2 \mathrm{M} \mathrm{NaOH}$ was added to the reaction mixture, which was then held at $80{ }^{\circ} \mathrm{C}$ for 2 hours to form the free hydroxy acids by hydrolysis. The resulting solution was diluted to a final volume of $5 \mathrm{~mL}$ with distilled water. 10 $\mu \mathrm{L}$ aliquots of the mixture were then diluted with $190 \mu \mathrm{L}$ of an internal standard solution of 100 $\mu \mathrm{M}$ adipic acid. Samples were filtered through a 96-well MultiScreen ${ }^{\text {HTS }}$ plate for HPLC/MS analysis.

The method of HPLC- triple quadrupole MS analysis (QQQ) was developed in our previous study ${ }^{14}$. In detail, an Agilent 1290 HPLC equipped with an auto-sampler, Phenomenex RezexROA Organic Acid H+ column $(150 \times 4.6 \mathrm{~mm})$ (Torrence, CA), and Carbo-H+ Security Guard cartridge was used for components separation. $0.5 \% \mathrm{v} / \mathrm{v}$ formic acid at flow rate of $0.3 \mathrm{~mL} / \mathrm{min}$ was used as mobile phase with a column temperature of $55^{\circ} \mathrm{C}$, and hydroxy acids were quantified by mass spectrometry on an Agilent 6460 triple quadrupole MS with ESI source, operating in negative ion MRM transition mode with fragmentor voltage $70 \mathrm{~V}$. Between 5-8 
min, the following transitions and collision energies were monitored: $\mathrm{m} / \mathrm{z} 145.1 \rightarrow 83.1,10 \mathrm{~V}$ (adipic acid, internal standard); $\mathrm{m} / \mathrm{z} 131.1 \rightarrow 73.2,5 \mathrm{~V}$ (3-hydroxy-2-methylvaleric acid); m/z $117.1 \rightarrow 73.2,5 \mathrm{~V}$ (3-hydroxy-2-methylbutyric acid); m/z117.1 $\rightarrow 59.2,5 \mathrm{~V}$ (3-hydroxyvaleric acid) $; \mathrm{m} / \mathrm{z} 103.1 \rightarrow 59.2,5 \mathrm{~V}$ (3-hydroxybutyric acid). Samples were quantified relative to a standard curve of 19.5, 39, 78, 156.25, 312.5, 625, 1250, $2500 \mathrm{mg}$ L-1 hydroxyacids (Figure S12). Standards of 3-hydroxybutyrate was purchased from Sigma-Aldrich (St. Louis, MO), 3hydroxy-2-methylbutyric acid were purchased from Santa Cruz Biotechnology (Dallas, Texas). Standards of 3-hydroxypentanoate and 3-hydroxy-2-methylpentanoate were prepared in our previous study ${ }^{14}$. Standard curve was normalized for injection volume using the internal standard.

Characterization of branched polymer by Nuclear Magnetic Resonance (NMR) Spectroscopy. Purified PHA was dissolved in deuterated chloroform $\left(\mathrm{CDCl}_{3}\right)$ at a concentration of 10-30 mg/mL. Spectra were recorded on a Bruker Avance spectrometer operating at $800 \mathrm{MHz}$ using a TXI probe and at a temperature of $298 \mathrm{~K}$. Data were processed with instrument software (Topspin 2.1, Bruker Biospin Inc) and Mnova software (Mestrelab Research, Escondido, CA).

$1 D^{1} H N M R$ : One dimensional proton spectra were acquired with a 30 degree flip angle pulse (pulse sequence $\mathrm{zg} 30$ ). A total of $128 \mathrm{k}$ points were collected for each of 8 scans, with a recycle delay set to $10 \mathrm{sec}$, which was five times the longest T1 $(2 \mathrm{sec})$ for quantitation. The carrier was set to $5 \mathrm{ppm}$ and the spectral width was set to $10 \mathrm{ppm}$. The data were apodized $(1 \mathrm{~Hz})$ prior to Fourier transformation.

$1{ }^{13} C N M R$ : One dimensional carbon spectra were acquired with a 30 degree flip angle pulse and pulse gated ${ }^{1} \mathrm{H}$ decoupling (pulse sequence zgpg30). A total of $32 \mathrm{k}$ points were collected for each of 32 scans, with a recycle delay of $6 \mathrm{sec}$. The carrier frequencies were set to $102.7 \mathrm{ppm}\left({ }^{13} \mathrm{C}\right)$ and $5.0 \mathrm{ppm}\left({ }^{1} \mathrm{H}\right)$ with a ${ }^{13} \mathrm{C}$ spectral width of $238.8 \mathrm{ppm}$. High resolution $1 \mathrm{D}{ }^{13} \mathrm{C}$ spectra of the $\mathrm{CO}$ region were recorded with a 90 degree flip angle pulse (pulse program $\mathrm{zg}$ ) and without ${ }^{1} \mathrm{H}$ decoupling. A total of $32 \mathrm{k}$ complex points were collected for each of 400 scans, with a recycle delay of 6 seconds. The carrier frequency was set to 170 ppm and the spectral width was $29.85 \mathrm{ppm}$. In each case, the data were zero-filled to $128 \mathrm{k}$ points prior to apodization $(2 \mathrm{~Hz})$ and Fourier transformation.

HSQC: One-bond ${ }^{1} \mathrm{H}-{ }^{13} \mathrm{C}$ correlations were detected with an edited ${ }^{1} \mathrm{H}-{ }^{13} \mathrm{C}$ HSQC experiment using pulse sequence hsqcedetgpsisp2. The ${ }^{1} \mathrm{H}-{ }^{13} \mathrm{C}$ coupling constant was set to $145 \mathrm{~Hz}$. A total of $512\left({ }^{1} \mathrm{H}\right)$ and $64\left({ }^{13} \mathrm{C}\right)$ complex points were collected in the two dimensions, with spectral widths of $13.3 \mathrm{ppm}\left({ }^{1} \mathrm{H}\right)$ and $120 \mathrm{ppm}\left({ }^{13} \mathrm{C}\right)$ and carrier frequencies of $5.0 \mathrm{ppm}\left({ }^{1} \mathrm{H}\right)$ and $50 \mathrm{ppm}\left({ }^{13} \mathrm{C}\right)$. Four scans were signal averaged for each $\mathrm{T} 1$ increment, using a recycle delay of 2 seconds. NMR assignments are based on ${ }^{1} \mathrm{H}-{ }^{13} \mathrm{CHSQC}$ spectra and previous literatures ${ }^{15-16}$. 
Size Exclusion Chromatpgraphy (SEC) The average molar mass values and molar mass distributions of the PHAs were determined by SEC using a chloroform mobile phase on an Agilent 1100 series size-exclusion chromatograph with an HP 1047A differential refractive index detector through three Varian PLgel Mixed-C Columns at $35^{\circ} \mathrm{C}$ and a flow rate of $1 \mathrm{~mL}$ $\mathrm{min}^{-1}$. A polymer sample concentration of $3-4 \mathrm{mg} / \mathrm{mL}$ was used. Relative molar mass values were determined using ChemStation software (Aglient), calibrated using EasiCal polystyrene (PS) standards purchased from Agilent. SEC for Samples 7 and 9 were exclusively performed using the chloroform mobile phase. SEC on all other samples (1-6, and 8) were also performed in a tetrahydrofuran mobile phase on an Agilent Infinity 1260 series HPLC system equipped with a Wyatt HELEOS-II multiangle laser light scattering detector and a Wyatt Optilab T-rEX differential refractive index detector through three Wyatt Styragel HR columns at $25^{\circ} \mathrm{C}$ and a flow rate of $1 \mathrm{~mL} \mathrm{~min}^{-1}$.

Thermal Characterization of PHAs. Thermogravimetric analysis (TGA) to determine thermal stability of the PHAs was performed using a TA instruments Q500 Analyzer under nitrogen with a heating-ramp rate of $10{ }^{\circ} \mathrm{C} \mathrm{min}{ }^{-1}$ from room temperature to $550{ }^{\circ} \mathrm{C}$. A typical sample size was between 5 and $10 \mathrm{mg}$. Differential scanning calorimetry (DSC) measurements were conducted using a TA Instruments Discovery Series differential scanning calorimeter equipped with a liquid nitrogen cooling accessory. All samples (ca. 3-7 mg) were analyzed in hermetically sealed T-Zero aluminum pans under nitrogen atmosphere. A characteristic analysis methodology for the samples is as follows. The samples were first heated at a rate of $10{ }^{\circ} \mathrm{C}$ $\mathrm{min}^{-1}$ to $230^{\circ} \mathrm{C}$ to erase any thermal history (first heat cycle). The pan was then isothermally maintained at $230{ }^{\circ} \mathrm{C}$ for 2 minutes before cooling to $-50{ }^{\circ} \mathrm{C}$ at $10{ }^{\circ} \mathrm{C} \mathrm{min}{ }^{-1}$. The sample was then reheated from -50 to $230^{\circ} \mathrm{C}$ at the same rate (second heat cycle). All data was collected from the second heat cycle unless otherwise indicated. Values for the glass transition temperature $\left(T_{\mathrm{g}}\right)$ were acquired at the midpoint of each transition using Trios software, while the melting temperature $\left(T_{\mathrm{m}}\right)$ and crystallization temperature $\left(T_{\mathrm{c}}\right)$ were taken as the peak of the melting endotherm and peak of the crystallization exotherm respectively. The melting enthalpy $\left(\Delta H_{\mathrm{m}}\right)$ was calculated from the area of the endothermic melting peak, while the crystallization enthalpy $\left(\Delta H_{\mathrm{c}}\right)$ was calculated from the area of the exothermic crystallization peak. The $\Delta H_{\mathrm{m}}$ reported in Figure S16, has been corrected to take into account the enthalpy of crystallization of the samples, where $\Delta H_{\mathrm{m}}=\Delta H_{\mathrm{m}}$ (determined using the instrumental software) $-\Delta H_{\mathrm{c}}$. 


\section{Supplementary Data}

Table S1. Strains, plasmids, oligonucleotides, and sequences. (A) Strains and plasmids. (B) Oligonucleotides. (C) Gene sequences. (D) Protein sequences.

\section{A. Strains and plasmids}

\begin{tabular}{|c|c|c|}
\hline Strain & Description & Source \\
\hline BAP1 & $\begin{array}{l}F^{-} \text {ompT gal dcm lon hsdSB(rB- mB-) } \lambda(D E 3 \text { [lacl lacUV5-T7 gene1 } \\
\text { ind1 sam7 nin5) prpRBCDE (sfp (T7), prpE (T7))] }\end{array}$ & 1 \\
\hline $\mathrm{BAP} 1^{\mathrm{T} 1 \mathrm{R}}$ & Derived from BAP1, $\triangle$ fhuA::FRT, T1, and T5 Phage-resistant & This work \\
\hline $\mathrm{DH} 10 \mathrm{~B}^{\mathrm{T} 1 \mathrm{R}}$ & 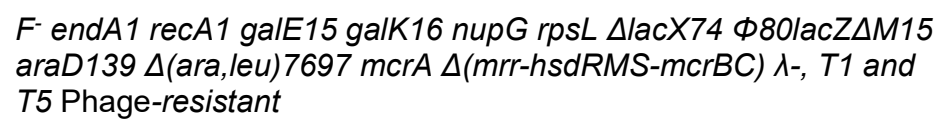 & Invitrogen \\
\hline $\mathrm{BL} 21(\mathrm{DE} 3)^{\mathrm{T} 1 \mathrm{R}}$ & $\begin{array}{l}\text { F- ompT gal dcm lon hsdSB(rB- mB-) } \lambda(\mathrm{DE} 3 \text { [lacl lacUV5-T7 } \\
\text { gene1ind1 sam7 nin5]), T1 and T5 Phage-resistant }\end{array}$ & Novagen \\
\hline Plasmid & Description & Source \\
\hline pAsAcat3-RePhaB & AsAcat3-RePhaB (Trc), lacl, $\mathrm{Cb}^{\mathrm{R}}, \mathrm{pBR} 322$ & This work \\
\hline pAsAcat3-CapPhaB0 & AsAcat3-CapPhaB0 (Trc), lacl, $\mathrm{Cb}^{\mathrm{R}}, \mathrm{pBR} 322$ & This work \\
\hline pAsAcat3-CapPhaB1 & AsAcat3-CapPhaB1 (Trc), lacl, Cb ${ }^{\mathrm{R}}, \mathrm{pBR} 322$ & This work \\
\hline pAsAcat2-CapPhaB2 & AsAcat2-CapPhaB2 (Trc), lacl, $\mathrm{Cb}^{\mathrm{R}}, \mathrm{pBR} 322$ & This work \\
\hline pAsAcat3-CapPhaB3 & AsAcat3-CapPhaB3 (Trc), lacl, $\mathrm{Cb}^{\mathrm{R}}, \mathrm{pBR} 322$ & This work \\
\hline pAsAcat3-AsHadh2 & AsAcat3-AsHadh2 (Trc), lacl, $\mathrm{Cb}^{\mathrm{R}}, \mathrm{pBR} 322$ & This work \\
\hline pAsAcat3-CaHbd & AsAcat3-CaHbd (Trc), lacl, $\mathrm{Cb}^{\mathrm{R}}, \mathrm{pBR} 322$ & This work \\
\hline pCapPhaB0A & CapPhaB0-CapPhaA (Trc), lacl, Cb ${ }^{\mathrm{R}}, \mathrm{pBR} 322$ & This work \\
\hline pRePhaA-RePhaB & RePhaA-RePhaB (Trc), lacl, Cb ${ }^{\mathrm{R}}, \mathrm{pBR} 322$ & This work \\
\hline pRePhaA-AsHadh2 & RePhaA-AsHadh2 (Trc), lacl, $\mathrm{Cb}^{\mathrm{R}}, \mathrm{pBR} 322$ & This work \\
\hline pRSF-CapMaoC1/2 & CapMaoC1/2 (T7), lacl, Km², RSF1030 & This work \\
\hline pT7-CapPhaC1 & CapPhaC1 (T7), Cm², p15a & This work \\
\hline pT7-CapPhaEC & CapPhaEC (T7), Cm², p15a & This work \\
\hline pT7-AcPhaPC* & AcPhaPC* (T7), Cm ${ }^{\mathrm{R}}, \mathrm{p} 15 \mathrm{a}$ & This work \\
\hline pT7-RePhaC & RePhaC (T7), Cm ${ }^{R}, \mathrm{p} 15 a$ & This work \\
\hline pT7-CapPhaEC-CpPct & CapPhaEC-CpPct (T7), Cm², p15a & This work \\
\hline pTesB2 & $\operatorname{TesB}(\mathrm{T} 7), \mathrm{Cm}^{\mathrm{R}}, \mathrm{p} 15 \mathrm{a}$ & Ref. 6 \\
\hline pET16b-CapPhaB0 & His-CapPhaBo (T7), lacl, Cb ${ }^{R}, \mathrm{pBR} 322$ & This work \\
\hline pET16b-RePhaB & His-RePhaB (T7), lacl, $\mathrm{Cb}^{\mathrm{R}}, \mathrm{pBR} 322$ & This work \\
\hline pET16b-RePhaB_Q150C & His-RePhaB_Q150C (T7), lacl, $\mathrm{Cb}^{\mathrm{R}}, \mathrm{pBR} 322$ & This work \\
\hline pHAS & His-RePhaC (T7), lacl, $\mathrm{Cb}^{\mathrm{R}}, \mathrm{pBR} 322$ & Ref. 17 \\
\hline pET28a-CapPhaEC & CapPhaE-CapPhaC-His (T7), lacl, KmR, pBR322 & This work \\
\hline pET28a-CapPhaE & CapPhaE-His (T7), lacl, Km R, pBR322 & This work \\
\hline
\end{tabular}




\section{B. Oligonucleotides}

\begin{tabular}{|c|c|}
\hline Primer & Sequence \\
\hline 82-Pct-1 & $\begin{array}{l}\text { AACGCATAATCTAGAGTCGAAAGATAAGGAGAGTAGAATATGCGTAAGGT } \\
\text { CCCGATTATC }\end{array}$ \\
\hline 84-Pct-3 & GCCAAGCTTGCATGCCTGCAтTAGСтTTTCAтCTCTTTCAGG \\
\hline 110-pPOL4.1 FWD & TGGTGATTGAGAAATTGTAAGAATTCAAGGAGATATATAATGACCCAGC \\
\hline 111-pPOL4.1 REV & TCGGTGAACTGGCTCGCCATTATATATCTCCTGGTCTGTTTCCTGTG \\
\hline 112-pCWori-A3AsH11 FWD & AACAGACCAGGAGATATATAATGGCGAGCCAGTTCACCGA \\
\hline 113-pCWori-A3AsH11 REV & 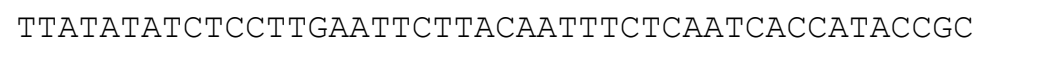 \\
\hline 114-pT7-TE3 FWD & САTATATTTTCCAACAGTAGTCTAGAGTCGACCTGCAGGC \\
\hline 115-pT7-TE3 REV & 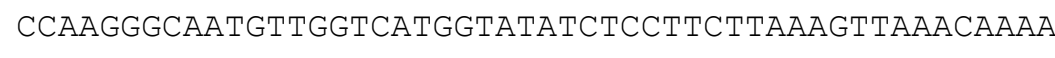 \\
\hline 116-pT7-TE3 FWD & TGCGCGAGCGCGGAGCCTGATCTAGAGTCGACCTGCAGGC \\
\hline 117-pT7-TE3 REV & TTAGGGTTTTTGTTTTCCACGGTATATCTCCTTCTTAAAGTTAAACAAАA \\
\hline 142-RBS-CapPhaB0 FWD & $\begin{array}{l}\text { ATTGAGAAATTGTAAGAATTGAAтTCTтAATCTCTAGGAGGTAAGTAтGC } \\
\text { AGATC }\end{array}$ \\
\hline 143-RBS-CapPhaB0 REV & $\begin{array}{l}\text { TAATTATACСTCCGGACTAGACTAGTTTACTTGGGTGCCATACGGATAGA } \\
\text { АС }\end{array}$ \\
\hline 144-RBS-CapPhaB1 FWD & $\begin{array}{l}\text { АTTGAGAAATTGTAAGAATTGAATTCCGTTATAAGGAGGAATATCTATGT } \\
\text { СССАAAA }\end{array}$ \\
\hline 145-RBS-CapPhaB1 REV & $\begin{array}{l}\text { TAATTATACCTCCGGACTAGACTAGTTCAATGCATGTGCAATCCTCCGTT } \\
\text { CA }\end{array}$ \\
\hline 146-RBS-CapPhaB2 FWD & $\begin{array}{l}\text { АTTGAGAAATTGTAAGAATTGAATTCCGTTATAAGGAGGAATATCTATGA } \\
\text { СTCAGC }\end{array}$ \\
\hline 147-RBS-CapPhaB2 REV & $\begin{array}{l}\text { TAATTATACCTCCGGACTAGACTAGTTTACTGGTAATACAAGCCCCCACA } \\
\text { GA }\end{array}$ \\
\hline 148-RBS-CapPhaB3 FWD & $\begin{array}{l}\text { АTTGAGAAATTGTAAGAATTGAATTCCGTTATAAGGAGGAATATCTATGA } \\
\text { СTСAАС }\end{array}$ \\
\hline 149-RBS-CapPhaB3 REV & $\begin{array}{l}\text { TAATTATACCTCCGGACTAGACTAGTTCAACTTACTAAAGTTTCCGAGGA } \\
\text { CA }\end{array}$ \\
\hline 150-pPOL4.1-AsAcat3 FWD & TTGCTATTGAGGTATTGTGAACTAGTCCGGAGGTATAATTAATGCTG \\
\hline 151-pPOL4.1-AsAcat3 REV & СTCCTAGAGATTAAGAATTCCTGTTTCCTGTGTGAAATTGTTATCCG \\
\hline 152-RBS-phaBA FWD & CACACAGGAAACAGGAATTCTTAATCTCTAGGAGGTAAGTATGCAGATC \\
\hline 153-RBS-phaBA REV & ААтTAтAсCтCCGGACTAGTTCACAATAсCTCAATAGCAATGGC \\
\hline 154-pT7-TE3 FWD & CGGTTGTCAAGTTACCCTAATCTAGAGTCGACCTGCAGGC \\
\hline 155-pT7-TE3 REV1 & $\begin{array}{l}\text { АСТTAАTGACGTTCATATTCATGGTATATCTCCTTCTTAAAGTTAAACAA } \\
\text { ААTTATTTCT }\end{array}$ \\
\hline 186-CapPhaB0 FWD & ССАтАTCGAAGGTCGTCATATGCAGATCAACGGAAGCGT \\
\hline 187-CapPhaB0 REV & GCTTTGTTAGCAGCCGGATCTTACTTGGGTGCCATACGGA \\
\hline 188-pT7-phaPC-F & $\begin{array}{l}\text { CCAACAGAGGAGGACGCCGCATGATAATCTAGAGTCGACCTGCAGGCATG } \\
\text { C }\end{array}$ \\
\hline 189-pT7-phaPC-R & $\begin{array}{l}\text { GCATGCCTGCAGGTCGACTCTAGATTATCATGCGGCGTCCTCCTCTGTTG } \\
\text { G }\end{array}$ \\
\hline 190-pCWori-A3AsH11 FWD & $\begin{array}{l}\text { GAAАTTGTAAGAАTTGAATTCTAAACCGTTATAAGGAGGAATATCTATGT } \\
\text { CCGCACTGC }\end{array}$ \\
\hline 191-pCWori-A3AsH11 REV & ATACCTCCGGACTAGACTAGTTACGCCGGCATACGCAGAG \\
\hline 288-pT7-CAPphaC1 FWD & ACGTCAAAGCCAAGGCATGATCTAGAGTCGACCTGCAGGCATGC \\
\hline
\end{tabular}




\begin{tabular}{|c|c|}
\hline 289-pT7-CAPphaC1 REV & $\begin{array}{l}\text { GCGCCTTTGCCGGTCGCCATGGTATATCTCCTTCTTAAAGTTAAACAAAA } \\
\text { TTATTTCTAGAGGGGA }\end{array}$ \\
\hline 290-pPOL1 FWD & CTTTAAGAAGGAGATATACCATGGCGACCGGCAAAGGCGC \\
\hline 291-pPOL1 REV & GCCTGCAGGTCGACTCTAGATCATGCCTTGGCTTTGACGTATCGC \\
\hline 675-fhuA-kan-F & $\begin{array}{l}\text { TCGTTTACGTTATCATTCACTTTACATCAGAGATATACCATGTAGGCTGG } \\
\text { AGCTGCTTC }\end{array}$ \\
\hline 676-fhuA -kan-R & $\begin{array}{l}\text { TTAGAAACGGAAGGTTGCGGTTGCAACGACCTGACGTTCTTGGGAATTAG } \\
\text { CCATGGTCC }\end{array}$ \\
\hline 677-fhuA-test-F & TCAGCCGCAGCAACAGCAACCTGCTCAGC \\
\hline 678-fhuA-test-R & CTTCTGACGGAGGCTGATGACGGCCAAGC \\
\hline 679-pA3B FWD & CCATATCGAAGGTCGTCATATGACCCAGCGCATCGCTTACG \\
\hline 680-pA3B REV & GCTTTGTTAGCAGCCGGATCCTTAGCCCATGTGCAGGCCACC \\
\hline 711-pA3B FWD & $\begin{array}{l}\text { TGGCTGTCGAACGCAAATAAGAATTCAAGGAGATATATAATGACCCAGCG } \\
\text { CA }\end{array}$ \\
\hline 712-pA3B REV & ATGTATATCTCCTTGAGCTCTCTGTTTCCTGTGTGAAATTGTTATCCGCT \\
\hline 713-pT5T33-phaA.HBD-crt FWD & $\begin{array}{l}\text { AATTTCACACAGGAAACAGAGAGCTCAAGGAGATATACATATGACTGATG } \\
\text { TTGTG }\end{array}$ \\
\hline 714-pT5T33-phaA.HBD-crt REV & TTATATATCTCCTTGAATTCTTATTTGCGTTCGACAGCCAGTGCT \\
\hline 715-pA3H2 FWD & $\begin{array}{l}\text { TGGCTGTCGAACGCAAATAAGAATTGAATTCTAAACCGTTATAAGGAGGA } \\
\text { ATATCTATGT }\end{array}$ \\
\hline 716-pA3H2 REV & ATGTATATCTCCTTGAGCTCCTGTTTCCTGTGTGAAATTGTTATCCGCTC \\
\hline 717-pT5T33-phaA.HBD-crt FWD & $\begin{array}{l}\text { CAATTTCACACAGGAAACAGGAGCTCAAGGAGATATACATATGACTGATG } \\
\text { TTGTG }\end{array}$ \\
\hline 718-pT5T33-phaA.HBD-crt REV & AACGGTTTAGAATTCAATTCTTATTTGCGTTCGACAGCCAGTGC \\
\hline 721-CapPhaE FWD & TAAGAAGGAGATATACCATGGAAAACAAAAACCCTAATGAGCAGCTTGGT \\
\hline 722-CapPhaE REV & GTGGTGGTGGTGGTGCTCGAGCTCCGATTTAGCGCGTGCAGC \\
\hline 725-CAPphaEC FWD & $\begin{array}{l}\text { TAAGAAGGAGATATACCATGGAAAACAAAAACCCTAATGAGCAGCTTGGT } \\
\text { GGG }\end{array}$ \\
\hline 726-CAPphaEC REV & GTGGTGGTGGTGGTGCTCGAGGGCTCCGCGCTCGCGCATCC \\
\hline 122-pRSFDuet-1 FWD & CTGTCGTCGCTTTAGCATGATTAACCTAGGCTGCTGCCAC \\
\hline 123-pRSFDuet-1 REV & $\begin{array}{l}\text { CAATGTGTTTTTTATCAATAAGCATATGTATATCTCCTTCTTATACTTAACTAAT } \\
\text { ATACT }\end{array}$ \\
\hline CAPmaoC1-maoC2 FWD & GTATAAGAAGGAGATATACATATGCTTATTGATAAAAAACACATTGGG \\
\hline CAPmaoC1-maoC2 REV & GTGGCAGCAGCCTAGGTTAATCATGCTAAAGCGACGACAG \\
\hline 763-499hbd-F & $\begin{array}{l}\text { GAAATTGTAAGAATTGAATTCAAGGAGATATATAATGAAAAAGGTTTGCGTTATT } \\
\text { GGTGC }\end{array}$ \\
\hline 764-499hbd-R & ATACCTCCGGACTAGACTAGTTACTTGGAGTAATCGTAAAAACCTTTGCCAGA \\
\hline CapPhaC1 FWD & CTTTAAGAAGGAGATATACCATGACCAACATTGCCCTTGG \\
\hline CapPhaC1 REV & GCCTGCAGGTCGACTCTAGACTACTGTTGGAAAATATATGTGCC \\
\hline CapPhaEC3 FWD & $\begin{array}{l}\text { CTTTAAGAAGGAGATATACCGTGGAAAACAAAAACCCTAATGAGCAGCTT } \\
\text { GGT }\end{array}$ \\
\hline CapPhaEC3 REV & GCCTGCAGGTCGACTCTAGATCAGGCTCCGCGCTCGCGCAT \\
\hline
\end{tabular}

\section{Gene sequences}

\begin{tabular}{l} 
AsAcat3 \\
ATGGCGAGCCAGTTCACCGACGTTGTGTTTGTGGGTGCGGCGCGTACCCCGGTTGGCTCTGTTCGTTCCTCCCT \\
GAGCACCGTGCCGGCGACGGTCCTGGGTGCCGAAGCGATCAAGGGCGCACTGAAGCACGCAAATCTGAAACCGA \\
\hline
\end{tabular}


GCCAGGTCCAGGAAGTCTTTTTCGGTTGCGTGGTTCCGAGCAATTGTGGCCAGGGTCCGGCTCGCCAAGCGACG CTGGGTGCTGGCTGCGATCCGAGCACGATTGTGACGACCCTGAATAAGCTGTGTGCAAGCGGTATGAAAAGCAT CGCTTGCGCGGCGAGCCTGTTGCAATTGGGTCTGCAAGAGGTGACCGTTGGTGGTGGTATGGAGAGCATGAGCC TGGTCCCGTACTATCTGGAGCGCGGTGAAACGACCTACGGTGGTATGAAACTGATTGATGGTATCCCGCGTGAC GGTTTGACCGATGCGTACAGCAACCAACTGATGGGTGCGTGTGCGGATAATGTCGCGAAACGTTTTAACATCAC CCGCGAAGAACAAGACAAGTTCGCAATTGAGAGCTACAAGCGCAGCGCGGCAGCATGGGAAAGCGGCGCGTGCA AGGCGGAGGTGGTGCCGATCGAAGTGACCAAAGGTAAAAAAACGTATATCGTTGATAAAGATGAGGAATACACC AAGGTGAATTTCGAGAAACTGCCGAAACTGAAACCGGCATTTCTGAAGGATGGTACGATTACCGCTGGTAACGC GAGCACGCTGAACGACGGTGCGGCAGCGGTCGTTATGACCACGGTGGAGGGTGCAAAAAAATACGGTGTTAAGC CGCTGGCGCGTCTGCTGAGCTATGGTGACGCCGCAACGAACCCGGTGGATTTCGCGATTGCTCCGAGCATGGTT ATTCCGAAGGTGTTGAAGCTGGCGAATCTGGAGATTAAAGACATTGATCTGTGGGAGATCAACGAAGCATTCGC CGTCGTTCCGCTGCACAGCATGAAGACCCTGGGTATCGATCATAGCAAAGTCAATATCCACGGTGGTGGTGTGT CCCTGGGCCACCCGATCGGCATGAGCGGTGCTCGTATCATCGTTCACCTGATCCACGCATTGAAACCGGGTCAA AAGGGCTGTGCGGCAATTTGCAATGGCGGCGGCGGTGCAGGCGGTATGGTGATTGAGAAATTGTAA

\section{CapPhaA}

ATGTCAGAGTCAGTCGTGATCGTAGGGGCTAAACGTACACCCGTTGGTGCGTTCCAAGGACAGTTTGCCGGGGT GACTGCTCCTCAGTTGGGGGCCGTGGCAATCAAAGCCGCGGTCGAACAGGCAGGTGTAAAAGGCGAAGACATTG ATGAAGCTCTTATGGGCTGCTGCCTTATGGCGGGTTTACGCCAGGCGCCCGCACGCCAGGCGGTGCTTGGGGCC GGATTACCGAAAAGTGTGCCTTGCACGACCTTAACAAAAATGTGTTCGTCGGCCCAAAAGACTGTCATGATTGC TCATGATGAGTTACTGGCGGGTAGCATTAACATCGCCATCGCCGGCGGAATGGAATCAATGACCAATGCCCCAC ACGTACTTACCACGGCTCGTACCGGCTACCGCTTGGGAAATGGTGTGCTTCACGACCATATGTTCCTTGATGGA CTTGAAGATGCTTATGAGCAAGGCAAACCTATGGGCGTTTTCGCAGAATTGTGCGTAGATAAATACGCCTTCTC ACGCGAAGAGATGGACGCTTATGCAGTTGAATCGGTTACGCGTGCGCAGAACAGTGTTGCGGCTGGTGTGTTTA AGGATGAGATCGCTCCTGTAACCGTCTCAACACGCAAAGGGGATGTTGTGTTCGACTCAGATGAGACCCCTATG AAGTGCGATGCTTCCAAGATCAGCAAATTGAAGCCTGTGTTCAAAAAAGATGGGGCTGTAACTCCGGCCAACTC CTCCTCGATTTCCGACGGAGCTGCGGCGTTTGTGTTAATGCGTGAGTCGGATGCTAAATCAGCGGGGATCACAC CCCTGGCGCGCATTGTGGGACACTCGACCTTCGCCCATGATCCTTCCTGGTTTACCACTGCTCCCGTGTTTGCG TTCCAGAAACTGCTTGCTAAGTTAGGATGGCAAGCAGAGGACGTAGACTTGTGGGAAATCAATGAAGCGTTTGC AGCAGTAACAATGGCTTCCATGCGTGATCTTAAGCTGCCTGCCGATAAAGTAAATGTTAATGGCGGCGCCTGCG CGCTGGGACACCCTATCGGTGCCACCGGATCGCGTATTCTGGTCACATTACTTTATGCTCTGAAAGCGCGTGGA TTGAAGCGTGGAATCGCCGGTTTGTGTGCGGGAGGCGGCGAAGCAACGGCCATTGCTATTGAGGTATTGTGA

\section{RePhaA}

ATGACTGATGTTGTGATTGTAAGCGCTGCACGTACTGCTGTTGGTAAGTTCGGTGGCTCCCTGGCAAAAATTCC GGCTCCGGAACTGGGCGCAGTAGTTATCAAGGCGGCACTGGAGCGTGCCGGTGTGAAACCGGAACAAGTGAGCG AGGTAATTATGGGTCAAGTGCTGACTGCGGGTAGCGGTCAAAACCCGGCTCGTCAGGCGGCCATCAAAGCAGGT CTGCCGGCTATGGTGCCGGCGATGACGATCAACAAAGTTTGTGGCTCTGGCCTGAAAGCTGTGATGCTGGCGGC TAACGCGATTATGGCAGGCGACGCCGAAATTGTCGTTGCTGGTGGTCAGGAGAATATGTCTGCGGCACCGCATG TTCTGCCGGGTTCCCGTGATGGCTTCCGCATGGGTGATGCAAAACTGGTTGACACTATGATCGTCGATGGTCTG TGGGACGTATACAACCAGTACCACATGGGTATCACTGCCGAAAACGTTGCTAAAGAGTATGGCATCACCCGCGA AGCCCAAGATGAATTTGCAGTCGGCTCTCAAAACAAAGCGGAAGCGGCGCAGAAAGCTGGCAAATTCGATGAGG AGATTGTTCCGGTTCTGATTCCACAGCGCAAAGGCGACCCGGTGGCCTTTAAAACCGACGAGTTTGTGCGTCAA GGCGCAACCCTGGATTCTATGTCCGGTCTGAAACCGGCCTTCGACAAAGCTGGTACTGTTACTGCGGCGAACGC AAGCGGTCTGAACGATGGTGCGGCGGCAGTTGTGGTTATGAGCGCGGCAAAAGCAAAAGAACTGGGTCTGACCC CGCTGGCCACCATCAAATCTTACGCTAACGCGGGCGTTGACCCGAAAGTGATGGGCATGGGCCCGGTTCCGGCG TCTAAACGCGCACTGAGCCGTGCGGAGTGGACTCCGCAGGACCTGGATCTGATGGAAATTAACGAAGCATTTGC GGCTCAAGCACTGGCCGTTCATCAGCAGATGGGCTGGGACACCAGCAAAGTTAACGTTAATGGTGGTGCAATCG CTATTGGCCACCCAATCGGTGCTTCTGGTTGCCGTATCCTGGTGACGCTGCTGCATGAAATGAAACGCCGTGAT GCTAAAAAGGGCCTGGCAAGCCTGTGCATTGGTGGTGGTATGGGTGTAGCACTGGCTGTCGAACGCAAATAA

\section{RePhaB}

ATGACCCAGCGCATCGCTTACGTAACCGGTGGCATGGGTGGTATTGGCACCGCAATCTGTCAGCGTCTGGCCAA AGACGGCTTTCGTGTTGTTGCGGGTTGCGGTCCGAACTCTCCGCGCCGTGAAAAATGGCTGGAACAGCAAAAAG CGCTGGGTTTTGACTTCATCGCAAGCGAAGGTAATGTTGCGGACTGGGATTCTACCAAGACCGCATTCGACAAA GTTAAATCTGAAGTAGGCGAGGTAGATGTCCTGATCAACAACGCAGGTATTACCCGTGATGTTGTGTTCCGTAA AATGACGCGTGCTGACTGGGACGCGGTGATCGATACCAACCTGACTTCCCTGTTTAACGTGACGAAACAAGTTA TTGACGGTATGGCTGACCGCGGTTGGGGCCGTATCGTGAATATCTCTTCCGTGAACGGCCAAAAAGGTCAATTC GGCCAGACTAACTACTCTACCGCGAAAGCAGGTCTGCACGGTTTTACTATGGCGCTGGCTCAGGAAGTTGCGAC 
TAAAGGTGTTACGGTTAACACCGTATCTCCGGGTTACATTGCTACTGATATGGTTAAAGCTATTCGTCAGGATG TTCTGGACAAGATCGTCGCCACGATCCCGGTGAAGCGCCTGGGCCTGCCGGAGGAAATCGCAAGCATCTGTGCA TGGCTGTCTTCCGAAGAATCCGGTTTCTCCACGGGTGCAGACTTTTCCCTGAACGGTGGCCTGCACATGGGCTA A

\section{CapPhaBo}

ATGCAGATCAACGGAAGCGTGTTTGTCGTGACGGGGGCCGGCAGCGGCTTGGGCGCTGCTACCGCGTCTAATAT CGTCAATGGTGGGGGAAAAGTTGTAATCGTCGATGTCGATGCAGGCGCCGGAGAAGCACAGGCTGCACAGTTGG GTGCCAATGCCCGCTTCGCAAAAACTGACGTGACCGACGAAGCGTCGGCTCGCGCTGCAATCGACTTAGCCGTC AGTGAATTCGGCGCACTTCACGGACTTGTCAACTGCGCGGGTGTCGCTCCACCGAAGAAGGTATTGGGTCGCGA TGGTCCCCACGACTTGGCAACATTTGCTCGTGTTGTATCGATCAATCTGGTCGGGACTTTCAATATGATCCGCC TTGCCGCCGAGGCTATGAGCAAAAACGAGCCTAATGAAGGGGGCGAGCGCGGCGTGATCGTGAATACTGCGTCT GTCGCCGGATACGACGGACAGATTGGTCAAGCCGCCTATGCCAGCAGTAAAGCCGGTGTCATTGGACTTACTTT GCCTGTCGCACGCGAACTTGCTGCCCACGGCATTCGCGTCGTAACCATCGCACCGGGGATCTTCGAGACACCGA TGCTTAAAGGCTTACCCCAAGCCGCTCAAGATAGCTTGGGTAAAATGGTCCCCTTTCCGTCTCGCCTTGGAAAA CCCGCCGAGTATGCTGCGTTAGTCCAACATATTTGCGAGAACGGCTATCTGAACGGAGAGGTGATTCGCCTTGA CGGTTCTATCCGTATGGCACCCAAGTAA

\section{CapPhaB1}

ATGTCCCAAAAGGTTGCCTACGTCACTGGCGGCATGGGTGGCATCGGGACGGCCATTTGTCAACGCTTACACGA CGACGGGTTTAAGGTGATCGCCGGTTGTGGACCCACCCGTGACTTCCAAAAGTGGCTTGATGAGCAGAAAGCCC TGGGATACACGTTCTATGCTAGTGTTGGGAATGTCGGTGATTGGGCATCTACGGTCGAGGCTTTCGGTCAAGCA AAACAAGAACACGGCTCCATCGACGTGTTAGTCAATAACGCAGGCATCACGCGTGATCGCATGTTTTTGAAGAT GACACCTGACGACTGGCAGGCCGTGATCGAGACGAATCTTAACTCTATGTTCAACGTCACAAAGCAAGTAGTCG GGGATATGGTTGAGAAGGGGTGGGGACGCGTGATCAACATTAGTAGCGTGAATGGTGCTAAGGGCCAGGCCGGT CAGACGAATTATTCCGCCGCGAAGGCGGGAATGCATGGCTTTACCATGGCCCTGGCCCAGGAATTGGCCTCCAA GGGAGTGACCGTAAATACTGTATCTCCCGGATATATTGGCACTGATATGGTAAAAGCAATCCGTCAGGATGTGC TGGATAAAATTGTTGGCACGATCCCAGTTAAACGCCTGGGGGAACCCCGCGAGATTGCTAGCATCGTGTCATGG CTGGCGAGTGAGGATGGGTCATACAGCACTGGTGCAGACTTTTCTGTGAACGGAGGATTGCACATGCATTGA

\section{CapPhaB2}

ATGACTCAGCGCGTAGCACTGGTTACCGGGGCGATGGGAGGAATTGGAACTGCTATCTGTCAAGAACTTGCAAA GGCGGGACACAAAGTAGTCGCAGCATACCATCCCGAATTCGATAAGCCTGAAGAATGGACAAAGGCAATGGCCG AGGCAGGGTTTAACGACTTTATCTGTGTTGCAGGCGATGTGTCGGATTATGATTCCTGTGTGGCGCTGACTGCG GAAGCAGAGGCTAAGGCCGGACCTATTGATATTCTTGTGAATAACGCAGGCATCACGCGCGATAAAATGTTCGC ACGTATGGAGCTTGCGCAGTGGAACGCCGTCATTAGTACAAATCTGAGCTCCCTGTTCAACATGACCAAGCAAG TATCGGCCAAGATGGCAGAACGCGGGTGGGGCCGTATCATTAACATTAGTTCTCTTAATGGCCTGAAAGGGCAG GCCGGACAAACGAATTATTCAGCAGCTAAGGCGGGCGTAATCGGGTTTACTAAGGCCCTTGCGGCGGAAGTGGC TGCAAAGGGTGTCACAGTAAATGCAATCTGTCCCGGTTATGTGGCTACTCCGATGGTTATGGCTATCAAGCCGG AGATTTTACAAGGGATTGTGGATACAGTCCCGATGAAACGTCTGGCGAAACCTGAAGAGATCGGGGGAGCCTGC GCGTACTTAGCTAGCGACATCGCAGGCTTTATGACGGGCAGTACGATGAATATCTGTGGGGGCTTGTATTACCA GTAA

\section{CapPhaB3}

ATGACTCAACGTGTAGCACTTGTAACCGGAGCTATGGGCGGTATTGGAACTGCTGTCTGTCAAGAGTTAGCTAA AGCAGGGCACAAAGTCGTGGCCGCCTACCATCCTCAATTTGATAATAAAGACGCTTGGTTGGCCGAGCAGGAGG CCGCCGGGTTTAAAGATTTTGTTTGTGTCGCCGGGGATGTCGCCGATATCGCCAGTTGCGAGGCTATGGTCAAG GAAGCGGAGGAAAAAGCAGGACCAGTAGACATTCTGGTGAACAACGCTGGGATCACTCGTGATCGTATGTTTGG AAAAATGGAAAAAGACCAGTGGGATGCGGTAATTAGTACCAACCTGTCTTCGCTGTTCAACATGACGAAACAGG TGAGCAACAAAATGGCTGAACGTGGATGGGGCCGCATTGTGAATATTTCTTCTGTAAACGGGGTTAAGGGACAA GCGGGGCAAACCAACTATAGTGCGGCGAAAGCTGGTGTAATCGGTTTTACGAAAGCCCTGGCAGCCGAGTTAGC CGCAAAAGGTGTCACGGTAAACGCAATCGCCCCCGGATACATTGCCACTAAAATGGTGATGGCGATCCGTGAGG ATGTTCTGAAGGGTATCGTAGACACTGTACCTATGAAACGCTTGGGTAAGCCCGAGGAAATGGGAGCAGCAGTG GTCTATCTGTCCTCGGAAACTTTAGTAAGTTGA

\section{AaHadh2}

ATGTCCGCACTGCGCTCCACGAAAGGTCTGGTCGCGCTGGTGACGGGTGGTGCGAGCGGCCTGGGCCGTGGTGC CGCTGAAAATCTGCTGAAACACGGCGCAAAAGTGGCTATTCTGGATCTGCCGAGCAGCGCGGGCGCAGAGGTTG CGAAAGAGCTGGGTGGTGATTGCATTTTTACCCCGGCAAGCGTCACGGCAGCAAGCGAAGTTAAGAGCGCCCTG GCGGATGTTAAGAAAAAATTTGGTCGTTTGGACGTTGCGGTGAACTGCGCGGGTATTGCGTACAGCTTTAAACT GTTCAACGTGAAGAAGAAAAAACTGTGCGATCTGGAAAGCGTGCGTAAAACGCTGGATGTCAACGTCATGGGCT 
ACTTCACGGTTGCGGCTCACGCAGCGGAGCTGTTCGCGGAGAATGAGAAAGACGAGATGGGCCAACGCGGTGTT ATTATTAATACCGCAAGCATTGCAGCGTTCGACGGTCAAGCCGGCCAAAGCGCGTATAGCGCCTCCAAGGGCGC GATTGTTGGCATGACCCTGCCGCTGGCCCGCGACTTCGCCGACGATGGCATCCGCGTTGTGACCATCGCTCCGG GCATCTTTGACACCCCGATGATGGCGTCCTTCCCGGACAAAGTTCGTAACTTCCTGATTGGTTTGGTGCCGAAT CCGAAGCGTTTCGGTGTGCCGGAGGAATACGGCGCTCTGGTTCGTCATATCATCGAAAACCGCTACCTGAACGG CGAGGTTATTCGTCTGGATGGTGCTCTGCGTATGCCGGCGTAA

\section{RePhaC}

ATGGCGACCGGCAAAGGCGCGGCAGCTTCCACGCAGGAAGGCAAGTCCCAACCATTCAAGGTCACGCCGGGGCC ATTCGATCCAGCCACATGGCTGGAATGGTCCCGCCAGTGGCAGGGCACTGAAGGCAACGGCCACGCGGCCGCGT CCGGCATTCCGGGCCTGGATGCGCTGGCAGGCGTCAAGATCGCGCCGGCGCAGCTGGGTGATATCCAGCAGCGC TACATGAAGGACTTCTCAGCGCTGTGGCAGGCCATGGCCGAGGGCAAGGCCGAGGCCACCGGTCCGCTGCACGA CCGGCGCTTCGCCGGCGACGCATGGCGCACCAACCTCCCATATCGCTTCGCTGCCGCGTTCTACCTGCTCAATG CGCGCGCCTTGACCGAGCTGGCCGATGCCGTCGAGGCCGATGCCAAGACCCGCCAGCGCATCCGCTTCGCGATC TCGCAATGGGTCGATGCGATGTCGCCCGCCAACTTCCTTGCCACCAATCCCGAGGCGCAGCGCCTGCTGATCGA GTCGGGCGGCGAATCGCTGCGTGCCGGCGTGCGCAACATGATGGAAGACCTGACACGCGGCAAGATCTCGCAGA CCGACGAGAGCGCGTTTGAGGTCGGCCGCAATGTCGCGGTGACCGAAGGCGCCGTGGTCTTCGAGAACGAGTAC TTCCAGCTGTTGCAGTACAAGCCGCTGACCGACAAGGTGCACGCGCGCCCGCTGCTGATGGTGCCGCCGTGCAT CAACAAGTACTACATCCTGGACCTGCAGCCGGAGAGCTCGCTGGTGCGCCATGTGGTGGAGCAGGGACATACGG TGTTTCTGGTGTCGTGGCGCAATCCGGACGCCAGCATGGCCGGCAGCACCTGGGACGACTACATCGAGCACGCG GCCATCCGCGCCATCGAAGTCGCGCGCGACATCAGCGGCCAGGACAAGATCAACGTGCTCGGCTTCTGCGTGGG CGGCACCATTGTCTCGACCGCGCTGGCGGTGCTGGCCGCGCGCGGCGAGCACCCGGCCGCCAGCGTCACGCTGC TGACCACGCTGCTGGACTTTGCCGACACGGGCATCCTCGACGTCTTTGTCGACGAGGGCCATGTGCAGTTGCGC GAGGCCACGCTGGGCGGCGGCGCCGGCGCGCCGTGCGCGCTGCTGCGCGGCCTTGAGCTGGCCAATACCTTCTC GTTCTTGCGCCCGAACGACCTGGTGTGGAACTACGTGGTCGACAACTACCTGAAGGGCAACACGCCGGTGCCGT TCGACCTGCTGTTCTGGAACGGCGACGCCACCAACCTGCCGGGGCCGTGGTACTGCTGGTACCTGCGCCACACC TACCTGCAGAACGAGCTCAAGGTACCGGGCAAGCTGACCGTGTGCGGCGTGCCGGTGGACCTGGCCAGCATCGA CGTGCCGACCTATATCTACGGCTCGCGCGAAGACCATATCGTGCCGTGGACCGCGGCCTATGCCTCGACCGCGC TGCTGGCGAACAAGCTGCGCTTCGTGCTGGGTGCGTCGGGCCATATCGCCGGTGTGATCAACCCGCCGGCCAAG AACAAGCGCAGCCACTGGACTAACGATGCGCTGCCGGAGTCGCCGCAGCAATGGCTGGCCGGCGCCATCGAGCA TCACGGCAGCTGGTGGCCGGACTGGACCGCATGGCTGGCCGGGCAGGCCGGCGCGAAACGCGCCGCGCCCGCCA ACTATGGCAATGCGCGCTATCGCGCAATCGAACCCGCGCCTGGGCGATACGTCAAAGCCAAGGCATGA

\section{CapPhaC1}

ATGACCAACATTGCCCTTGGCCAATTGAGTGGAGGCGTATCACCTGCCTCGTTGGCGATGGCCTACCTGGATTG GATGGTCCACCTTTCGGCGTCCCCGGGTAAACAGTTTCAGCTGGGCGCTAAGGCAGCGCGTAAAGCCTTACGCT TGGGGTCCTATGCTCTTACATCGGCGGTTACGGGTAACGCAGAACCTTGTATCAAGCCGCTGCCGGGGGATCAC CGTTTCGACCACCCTGGATGGCAGCGTTTTCCTTATAATGTTATTTATCAGGGCTTCTTGCTTAATCAACAGTG GTGGCATAATGCGACAACTGGCGTTCGCGGTATTTCCAAACACTCTGAAGCGGCCGTTTGGTTTACAGCAAAAC AAATTCTGGATATGATGTCCCCCGTAAACGTCCCTGCAATGAATCCAGAAATCGTCGAGGCGACGGTAAAGGAA CGTGGAGCCAACCTTGCACGTGGAGCAGAATTCTATGGCGAGGATGTTCGCCGCAGCCTTCGCGATGAAAAGCC CGCTGGTATTGAGGCTTTTCGCGTTGGGGAAAATTTGGCCATCACGCCAGGAAAGGTCGTATTTCGCAACCACT TAATCGAATTGATCCAATATTCACCCAGTACAGATACTGTGCAACGCGAGCCGGTGTTAATGCAAAGCGCGTGG ATGATGAAGTACTACATCCTTGATCTTAGCCCTCACAATTCTCTGGTTAAATACCTTGTAGATCGCGGACATAC GGTGTTTATGATCAGCTGGTTGAACCCCGGTCCCGAACACCGCAATCTGGGGATGGAGGATTATCGCAAATCCG GGACGATGGCCGCCATTGATGCGATTAGCGAAATCCTTCCACAACGTAAGATCCACACGGTTGGGTATTGTCTT GGGGGTATCTTGCTTACCATCGCAGCGGCTGCAATGGCGCGTGACGGCGACGACCGTTTGGCCTCAGTTACTTT ATTCACTACTATGACTGACTTTACTGATGTCGGGGAGATCAATGTATTTATGGACGCATCGGAAGTCACACTGT TAGAGGATATGATGTGGCAGAAAGGGTATCTGGGACATAAGCAAGTATCAGGGGGGTTTCAATTACTGAAATCC GCGGATTTGATCTGGTCGAAAATGGTCCGTGAATACTACTTGGGGCATCGTGAGCCAATGTTTGATCTGATGGC ATGGAACGCAGACGGTACCCGTATGCCTTACCGCCAACACTCAGAGGTGCTTCGTCGCTTGTATGTCGATAATG AACTGTTTCAGGGGAAGTATATGGTCGGGGGACGCGCCGTGTCGATCTCAAACATTCACTGTCCTATGTTCGCA GTTGCCGCGGTAGCAGACCATGTAGCTCCTTGGCGTAGCGTATACAAGCTTCATTTGCAATCCGACTCTGCAGA GCTGACATTCGCCTTAACTTCCGGGGGGCATAACGTAGGTATTGTTAATGAACCAGGGCACCCCCGCCGCTCAT TTCAATTAAGTGTATGTCGTGAGGGAGAACGTTTCTTGGACGCCGAGACGTGGAAAGAACGCACTCCAAAATTT GAAGGGTCGTGGTGGCCCGTCTGGCAGGAGTGGTTAGTTCGCCATTCTTCTGGTGGTGAAGCCGCGCCACCGAT GGGTGCGCCGGAGAAGGGGTATACGGCCTTGTGCGATGCACCAGGCACATATATTTTCCAACAGTAG

\section{CapPhaEC (two genes)}


GTGGAAAACAAAAACCCTAATGAGCAGCTTGGTGGGGTTGTCAACGCATGGGCGGACATGCAGAAACGTATGTG GGGTGATTGGTCTTCCTTACTTCAAAATTTACCTGGGGGCTCTGAAGGTCCCGTGGAGGCGGCCAAGAAAGGAG TGGCGGCCGCATCGAAGGGAACTAATGAGGCGGCGCGCATGCTGATGGACCGCATGACCTCTTCTCAAGGTGCG ATGAACCGCGTTATGGATTTTTTTTTCAAGTCCATGAAAATCGTAGCACCCAACTTAGAGGCCAACAAAGACTG GCGTCCTGATCTTAAGGGTTTTGCGGAACAGTGGGCCAAAGAATCTACTGCGATGCTGGAACGTTCATTTGGGA TGGGGTCACATTTGGGGAATCTGAGTTCGACATTGAGCAAAGACTTACCAGACGCAATGGGACCTTGGCTGTCT TTTCTGATGCAAGCCGCAAGCTCCGGTCACGTGGGGGAGGCCATGTTAGGTGGAACCAGCGGGATCAATCGCCT TCTTTCTATGGAAGGCGACGCCGCCTTGGCAGGAGTTGGCGAAATCCCCCTTTTCGGAGCCTCACGTGAAAAAA ATGCCAAGTTGCTTCGTTTGGTTGATGCGGTCGTAGATCTTCGCAAGAATAGTTTAACATTCCACACCGCATTT GGTGATGCATTAGCTAAAGCGGTTGAGGCGACAGTGGAGGAACTTGGGAAAGTTGCTGCCAAGGGAGAAAAGAT TACTGCTGTTCGCCAGCTTATGTCGCTGTGGTACCGTACAGCGGACAAGTCATTGTTGGTGACGTTTAACACGC AAGAGTTCCTGGATAAACAAAACGCTTTTACGGCCGCACAACAACAGTTCAAATTGGCACAGCGCGCCGTGGTG GAGGATATTTTTCGTGGATTAGACATGCCTACGCGCAGCGAATTGGACGAGACATACCAAGTTATCCACGAGTT GAAAAAAGAGGTCCGTGCTTTAAAAAAAGCCCTGCTTCCAGCCGCACCCGCAGCAGTTGCCAAGTCTTCCCCCG CTCCCCGCAAGGCCGCAGCTGCACGCGCTAAATCGGAGTGAGCCGAGAAGCCTGAAATTCGTTATCCGGCTGAT AAGCTAATAGACCTTTAGGAGGGTGCTACCGATGTTTAGCTTTCCAATCCAGATTCTTCCGGCAGATGTCGCGG CTGAGACGGCTGCATTGAACGAGAAATTAGCGAAAGGCATCTCGAATCTTACCAACCTTACGGATGATGATATT GACATCGGTAGCACTCCGAAAGACGTAGTTTTCGAACAGGACGGAATCAAGGTCTACCATTATCACGCCCTTGC CGAACCTTCGCAAATCATGAAAACACCCCTGCTGATCGTCCCCCCGCTGATTAACGGTTACGAAGTCGCCGACT TACAGCCTGACCGCTCCCTTGTGCGCAATTTATTGAATCAGGGTATCGATGTGTATTTGAATGATTGGGGGTAC CCCCGTCAAGTGGATAAGTATCGCACTTTAGATGATTACATCAATGGTTACTTTGACGATACGGTGGATTTCAT CCGCAAACATCACGGCGTGGACAAAATCGCCCTTTTTGGCATTTGTCAAGGAGGCGCGATGTCAACTACTTACT CGACTCTTAATCCAGATAAGATCAGTCATCTGGTCTTAACCGTCAGCCCTATCGACTTTGACGCTTACAAAGCG AACCACAAGCCCCACGAAGGATTGATGTTCACGATGGGTGCTGACGCAGATGTCGAGAAGATGGTCGCAGTCCA CGGAAATGTCCCTGCAACCGTGCTTAACGAATCGTTCATGATGGCGAGTCCGTTCATTTTAAATTACGGGAAAT ACGCTGATGTAATTGATATCTTAGATGATCGCTTAGCTTTACAGAACTTTTTGCGTATGGAAAAGTGGCTTTTC GGTGGGCCGGATGCGGGCGGGCAAATGTTCAAGGAGTTTATCCGCGATTTTTTAAAGGGAAATAAGTTGGTTAA GGGGACCCTTGAGATCGGGGGACGCAAAGTAGATTTGAAAGAACTTACCATCCCGATCTTAAATATTTTCGCAG AGAAAGATCATATTGTCCCACCTCCATGCACGGTAGCTCTGGGGAAGCACGTTGGGTCTAAAGACTACACCGAG TTTGCCATTGCGACCGGTCACATTGGCATTTATACGGGTGGTTTGAGCCAAAAAGTGCTTGCTCCTACGGTTGG AAAATGGATGCGCGAGCGCGGAGCCTGA

\section{AcPhaPC* (two genes)}

ATGAATATGAACGTCATTAAGTCATTTACAGAGCAGATGCAAGGCTTTGCAGCGCCTCTGACTCGCTATAACCA GCTTTTAGCAAGTAACATTGAGCAGTTAACACGCCTGCAACTTGCTTCCGCCAATGCTTACGCGGAATTAGGAC TGAACCAGTTGCAAGCCGTCTCAAAGGTGCAAGACACCCAATCGTTGGCTGCGCTGGGGACCGTGCAATTAGAA ACCGCGAGTCAGTTATCGCGTCAAATGTTAGACGACATCCAGAAACTGAGTGCATTGGGCCAGCAATTCAAAGA AGAGCTGGATGTTTTGACCGCCGACGGTATCAAAAAAAGCACCGGGAAGGCTTGATAACCCCTGGCTGCCCGTT CGGGCAGCCACATCTCCCCATGACTCGACGCTACGGGCTAGTTCCCGCCTCGGGTGTGGGTGAAGGAGAGCACA TGTCTCAGCCGTCGTATGGACCTTTATTTGAGGCCCTTGCACACTACAACGACAAACTTCTTGCAATGGCGAAA GCCCAAACGGAACGTACCGCCCAAGCGTTGTTACAAACAAACCTGGATGACCTGGGTCAGGTATTAGAGCAGGG CAGCCAGCAACCTTGGCAGTTGATTCAAGCTCAGATGAATTGGTGGCAGGACCAACTTAAGTTGATGCAACATA CTCTGTTAAAGTCTGCGGGCCAACCGTCAGAGCCGGTCATTACGCCAGAGCGTTCCGATCGTCGCTTCAAGGCG GAAGCGTGGTCGGAGCAGCCAATTTACGACTACTTGAAGCAGTCTTACTTGCTGACTGCGCGCCACTTGTTAGC GTCTGTAGACGCCTTGGAGGGTGTACCCCAGAAGTCACGTGAACGCCTGCGTTTCTTTACTCGCCAGTACGTGT CGGCGATGGCCCCATCCAATTTCCTGGCGACCAATCCCGAACTGTTAAAATTGACTCTTGAGTCAGACGGCGGA CAGAATCTTGTGCGTGGCTTGGCCTTACTGGCCGAGGACTTAGAACGTTCGGCCGACCAATTGAACATCCGTCT GACCGATGAATCTGCCTTCGAACTTGGGCGTGATCTTGCCCTGACACCAGGTCGCGTAGTCCAACGTACGGAGC TGTACGAATTAATTCAGTACAGCCCGACCACAGAGACCGTCGGCAAAACGCCAGTGCTTATCGTACCCCCGTTC ATTAACAAGTACTACATTATGGATATGCGTCCACAAAATTCCCTTGTCGCGTGGTTAGTTGCGCAGGGACAGAC TGTTTTTATGATTTCCTGGCGTAATCCAGGAGTAGCCCAAGCGCAAATTGACTTGGACGATTATGTCGTAGATG GTGTCATTGCAGCTTTAGATGGAGTTGAGGCAGCCACCGGAGAGCGCGAAGTACATGGTATTGGCTACTGTATC GGCGGAACAGCTCTTTCGTTGGCGATGGGCTGGCTGGCTGCTCGCCGCCAGAAACAACGTGTTCGCACAGCTAC GTTATTCACTACGTTATTAGACTTCAGCCAACCCGGTGAGTTAGGAATCTTTATTCACGAACCTATTATTGCTG CTCTTGAGGCGCAAAACGAAGCTAAGGGTATTATGGATGGGCGTCAATTGGCCGTAAGCTTTAGCTTGTTGCGC GAGAACTCTCTGTACTGGAACTATTACATCGACTCGTACTTGAAGGGGCAGAGTCCTGTGGCTTTCGATTTGCT TCACTGGAACTCCGACTCTACGAACGTAGCTGGCAAAACTCATAACTCGTTATTACGCCGCTTATACTTGGAAA 
ACCAGTTGGTGAAGGGTGAACTTAAAATTCGCAATACCCGTATTGACCTGGGCAAGGTAAAGACTCCTGTTCTT CTTGTCTCGGCGGTCGATGACCATATTGCACTGTGGCAGGGAACTTGGCAGGGCATGAAATTATTTGGGGGTGA ACAACGCTTCCTTTTAGCAGAGTCGGGTCACATCGCTGGAATCATCAACCCCCCCGCTGCTAATAAATACGGTT TCTGGCACAATGGTGCCGAGGCTGAGTCCCCAGAATCCTGGTTGGCTGGCGCGACTCACCAAGGGGGATCATGG TGGCCAGAAATGATGGGGTTCATCCAGAATCGCGATGAAGGGAGCGAACCGGTGCCCGCTCGTGTCCCAGAAGA GGGATTAGCCCCCGCACCGGGGCATTATGTCAAGGTTCGTCTGAATCCCGTGTTTGCCTGCCCAACAGAGGAGG ACGCCGCATGATAA

\section{EcTesB}

ATGAGTCAGGCGCTAAAAAATTTACTGACATTGTTAAATCTGGAAAAAATTGAGGAAGGACTCTTTCGCGGCCA GAGTGAAGATTTAGGTTTACGCCAGGTGTTTGGCGGCCAGGTCGTGGGTCAGGCCTTGTATGCTGCAAAAGAGA CCGTCCCTGAAGAGCGGCTGGTACATTCGTTTCACAGCTACTTTCTTCGCCCTGGCGATAGTAAGAAGCCGATT ATTTATGATGTCGAAACGCTGCGTGACGGTAACAGCTTCAGCGCCCGCCGGGTTGCTGCTATTCAAAACGGCAA ACCGATTTTTTATATGACTGCCTCTTTCCAGGCACCAGAAGCGGGTTTCGAACATCAAAAAACAATGCCGTCCG CGCCAGCGCCTGATGGCCTCCCTTCGGAAACGCAAATCGCCCAATCGCTGGCGCACCTGCTGCCGCCAGTGCTG AAAGATAAATTCATCTGCGATCGTCCGCTGGAAGTCCGTCCGGTGGAGTTTCATAACCCACTGAAAGGTCACGT CGCAGAACCACATCGTCAGGTGTGGATCCGCGCAAATGGTAGCGTGCCGGATGACCTGCGCGTTCATCAGTATC TGCTCGGTTACGCTTCTGATCTTAACTTCCTGCCGGTAGCTCTACAGCCGCACGGCATCGGTTTTCTCGAACCG GGGATTCAGATTGCCACCATTGACCATTCCATGTGGTTCCATCGCCCGTTTAATTTGAATGAATGGCTGCTGTA TAGCGTGGAGAGCACCTCGGCGTCCAGCGCACGTGGCTTTGTGCGCGGTGAGTTTTATACCCAAGACGGCGTAC TGGTTGCCTCGACCGTTCAGGAAGGGGTGATGCGTAATCACAATTAA

\section{CpPct}

ATGCGTAAGGTCCCGATTATCACCGCGGATGAAGCTGCGAAACTGATTAAAGACGGTGACACGGTGACCACCAG CGGCTTCGTGGGCAACGCAATCCCGGAGGCATTGGATCGTGCAGTCGAAAAGCGTTTTCTGGAAACGGGTGAGC CGAAGAACATTACGTACGTGTATTGTGGTTCTCAAGGCAATCGTGATGGCCGCGGTGCGGAACACTTCGCACAC GAAGGCTTGCTGAAGCGCTATATCGCAGGCCACTGGGCGACCGTCCCGGCGCTGGGCAAAATGGCGATGGAAAA CAAAATGGAGGCTTATAATGTGTCTCAGGGCGCACTGTGCCACCTGTTTCGCGATATTGCGAGCCACAAACCGG GTGTTTTTACCAAGGTTGGTATCGGCACGTTTATTGACCCGCGCAATGGTGGTGGTAAGGTGAATGACATTACC AAAGAAGACATTGTCGAGCTGGTGGAAATCAAGGGCCAAGAGTATTTGTTCTATCCGGCATTTCCGATTCATGT CGCCCTGATCCGCGGTACGTATGCAGATGAAAGCGGCAATATTACCTTCGAGAAGGAGGTTGCTCCGCTGGAAG GCACGTCCGTCTGCCAGGCGGTCAAGAATAGCGGCGGCATTGTTGTTGTTCAAGTTGAACGTGTGGTTAAGGCT GGTACGCTGGACCCGCGCCACGTCAAAGTTCCGGGCATCTATGTCGATTACGTGGTCGTGGCGGACCCGGAAGA TCACCAGCAGTCCCTGGACTGCGAATACGATCCGGCACTGAGCGGCGAACACCGTCGCCCGGAGGTCGTCGGTG AACCGCTGCCGTTGAGCGCGAAGAAGGTGATCGGTCGCCGTGGTGCCATTGAGCTGGAGAAAGATGTGGCGGTT AACTTGGGCGTTGGTGCCCCGGAGTACGTTGCCAGCGTGGCAGATGAGGAGGGCATCGTCGATTTCATGACCCT GACCGCTGAGTCCGGTGCGATCGGTGGCGTGCCGGCAGGTGGTGTTCGCTTCGGCGCTTCCTATAACGCGGACG CCTTGATCGACCAGGGCTATCAGTTTGATTACTATGACGGCGGTGGTCTGGACCTGTGTTACCTGGGCTTGGCA GAGTGCGACGAGAAGGGCAATATCAACGTGAGCCGTTTCGGCCCGCGCATTGCAGGTTGCGGTGGTTTCATTAA TATCACGCAGAATACGCCGAAAGTGTTTTTTTGTGGCACCTTCACCGCTGGCGGTCTGAAGGTCAAAATTGAAG ACGGTAAGGTTATTATTGTGCAGGAAGGTAAGCAAAAAAAGTTCCTGAAGGCGGTGGAACAAATTACGTTCAAC GGTGACGTTGCCCTGGCAAATAAGCAGCAGGTCACCTATATCACCGAGCGCTGCGTTTTCTTGCTGAAAGAGGA CGGCTTGCACTTGTCTGAGATCGCGCCGGGCATTGATCTGCAAACGCAAATCCTGGATGTTATGGACTTCGCCC CGATCATTGACCGTGATGCTAATGGTCAAATTAAGCTGATGGATGCTGCACTGTTCGCGGAGGGTCTGATGGGC CTGAAAGAGATGAAAAGCTAA

\section{CapMaoC1/2 (two genes)}

ATGCTTATTGATAAAAAACACATTGGGATGAAGGTTCCCCCACATTCCGTAAATCTGACCGCATGGCAACTGAAGTGGTTTG CAAAGGCGACTGGGGAAACGAACCCCATCTACTTTGACGAGGCAGCCGCGCACAAAGCGGGGCTTCCAGGCGTGCTGGCTCC ACCGACCTTCTTCTTTTGCATGGACATGGATAAAGAACACCCGTTTGACTATTTAGAAACGATGGGATGTGACCTGATGAAA ATGCTGCACGGTGAGCAGAGTTTTACATATCATAAGCCTGTTTACAGTGGAGACGTGTTAGACTTCGACGGCGAGATCACTG ACATCTATGATAAGCGCAACGGAGCACTTCAGTTTGTGGTGAAAGAAGTCAAGGTACGTCGTCAGGGCGAGCTGGTGTGTGA TGTGCGCTCTGTCATGGTCATTCGCGCCTAGGGGAAAGTCGATGACTAAGCCAAACTTCGATGCTCTTAATGTGGGAGATGA GTTACCCTCCTTCACCACTGAACCCGTTTCGCGTTTAACCTTAGCTTTATATGCTACCGGCTCTGGGGACCATCATCCTCTG CACTTAGATCAAGACTATGTTCGTGCAAACGGGATTCCCGACGTATTTGCCCACGGAATGCTTGGGATGGCGTATTTGGGGC GCTTATTAACTCAATGGGTCCCACAGGCGGCCATCCGCTCCTTCGGCGTACGTTTCACGGCCATCACTCAGATCGGTGAGCG CATGGTATGCACTGGTAAAGTGGTTGAAAAGCTGGAGCAGGGCGGAGAAAAATGTGTCCGTTTAGAGTTGACTTGTGCCAAT GAACAAGGCGAAGCGAAGCATAAGGGAGACGCTGTCGTCGCTTTAGCATGA 


\section{Protein sequences}

\begin{tabular}{l}
\hline His $\mathbf{1 0}$-CapPhaB0 \\
MGHHHHHHHHHHSSGH IEGRHMQ INGSVFVVTGAGSGLGAATASNIVNGGGKVVIVDVDAGAGEAQAAQLGANARFAKTDVT \\
DEASARAAIDLAVSEFGALHGLVNCAGVAPPKKVLGRDGPHDLATFARVVS INLVGTFNMIRLAAEAMSKNEPNEGGERGVI \\
VNTASVAGYDGQ IGQAAYASSKAGVIGLTLPVARELAAHG IRVVTIAPGI FETPMLKGLPQAAQDSLGKMVPFPSRLGKPAE \\
YAALVQHICENGYLNGEVIRLDGS IRMAPK*
\end{tabular}

\section{His ${ }_{10} N-R e P h a B$}

MGHHHHHHHHHHSSGH IEGRHMTQRIAYVTGGMGGIGTAICQRLAKDGFRVVAGCGPNS PRREKWLEQQKALGFDF IASEGN VADWDSTKTAFDKVKSEVGEVDVLINNAGITRDVVFRKMTRADWDAVI DTNLTSLFNVTKQVIDGMADRGWGRIVNISSVNG QKGQFGQTNYSTAKAGLHGFTMALAQEVATKGVTVNTVSPGYIATDMVKA IRQDVLDKIVATI PVKRLGLPEEIAS ICAWLS SEESGFSTGADFSLNGGLHMG*

\section{His $_{10}$ N-RePhaB_Q150C}

MGHHHHHHHHHHSSGH IEGRHMTQRIAYVTGGMGGI GTAICQRLAKDGFRVVAGCGPNS PRREKWLEQQKALGFDF IASEGN VADWDSTKTAFDKVKSEVGEVDVLINNAGITRDVVFRKMTRADWDAVI DTNLTS LFNVTKQVIDGMADRGWGRIVNISSVNG QKGQFGCTNYSTAKAGLHGFTMALAQEVATKGVTVNTVSPGYIATDMVKAIRQDVLDKIVATI PVKRLGLPEEIAS ICAWLS SEESGFSTGADFSLNGGLHMG*

\begin{tabular}{|c|}
\hline $\begin{array}{l}\text { His } \\
\text { } \mathbf{N}-\mathbf{R e P h a C} \\
\text { MGSSHHHHHHSSG } \\
\text { IAPAQLGDIQQRY } \\
\text { RFAISQWVDAMSP } \\
\text { QYKPLTDKVHARP } \\
\text { GQDKINVLGFCVG } \\
\text { ANTFSFLRPNDLV } \\
\text { YIYGSREDHIVPW } \\
\text { LAGQAGAKRAAPA }\end{array}$ \\
\hline $\begin{array}{l}\text { CapPhaEC-His }{ }_{6} \text { C } \\
\text { CapPhaE } \\
\text { MENKNPNEQLGGVVNAWADMQKRMWGDWSSLLQNLPGGSEGPVEAAKKGVAAASKGTNEAARMLMDRMTSSQGAMNRVMDFF } \\
\text { FKSMKIVAPNLEANKDWRPDLKGFAEQWAKESTAMLERSFGMGSHLGNLSSTLSKDLPDAMGPWLSFLMQAASSGHVGEAML } \\
\text { GGTSGINRLLSMEGDAALAGVGEIPLFGASREKNAKLLRLVDAVVDLRKNSLTFHTAFGDALAKAVEATVEELGKVAAKGEK } \\
\text { ITAVRQLMSLWYRTADKSLLVTFNTQEFLDKQNAFTAAQQQFKLAQRAVVEDIFRGLDMPTRSELDETYQVI HELKKEVRAL } \\
\text { KKALLPAAPAAVAKSSPAPRKAAAARAKSE* }\end{array}$ \\
\hline $\begin{array}{l}\text { CapPhaC-His }{ }_{6} \mathbf{C} \\
\text { MFSFPIQILPADVAAETAALNEKLAKGISNLTNLTDDDIDIGSTPKDVVFEQDGIKVYHYHALAEPSQ IMKTPLLIVPPLIN } \\
\text { GYEVADLQPDRSLVRNLLNQGIDVYLNDWGYPRQVDKYRTLDDY INGYFDDTVDFIRKHHGVDKIALFGICQGGAMSTTYST } \\
\text { LNPDKISHLVLTVSPI DFDAYKANHKPHEGLMFTMGADADVEKMVAVHGNVPATVLNES FMMASPF I LNYGKYADVIDILDD } \\
\text { RLALQNFLRMEKWLFGGPDAGGQMFKEF IRDFLKGNKLVKGTLEIGGRKVDLKELTIPILNIFAEKDH IVPPPCTVALGKHV } \\
\text { GSKDYTEFAIATGHIGIYTGGLSQKVLAPTVGKWMRERGALEHHHHHH* }\end{array}$ \\
\hline $\begin{array}{l}\text { CapPhaE-His }{ }_{6} \text { C } \\
\text { MENKNPNEQLGGVVNAWADMQKRMWGDWSSLLQNLPGGSEGPVEAAKKGVAAASKGTNEAARMLMDRMTSSQGAMNRVMDFF } \\
\text { FKSMKIVAPNLEANKDWRPDLKGFAEQWAKESTAMLERSFGMGSHLGNLSSTLSKDLPDAMGPWLSFLMQAASSGHVGEAML } \\
\text { GGTSGINRLLSMEGDAALAGVGE I PLFGASREKNAKLLRLVDAVVDLRKNSLTFHTAFGDALAKAVEATVEELGKVAAKGEK } \\
\text { ITAVRQLMSLWYRTADKS LLVTFNTQEFLDKQNAFTAAQQQFKLAQRAVVEDIFRGLDMPTRSELDETYQVI HELKKEVRAL } \\
\text { KKALLPAAPAAVAKSSPAPRKAAAARAKSELEHHHHHH* }\end{array}$ \\
\hline
\end{tabular}


Figure S1. Comparison of two thiolases AsAcat3 and RePhaA. Production of both 3hydroxy acids and PHA polyesters was tested in E. coli BAP1 strains bearing the two-plasmid systems detailed below to compare the ability of AsAcat 3 from A. suum and RePhaA RePhaA from $R$. eutropha to incorporate $\alpha$-branching. Strains were cultured for $2 \mathrm{~d}$ at $30{ }^{\circ} \mathrm{C}$ in TB broth containing $2 \%(w / v)$ glucose, $0.2 \%(w / v)$ sodium propionate, and $0.418 \%(w / v)$ MOPS. Data are mean \pm s.d. of biological replicates $(\mathrm{n}=3$ ). (A) Pathway of 3-hydroxy acids production. (B) Pathway of PHA polyesters. (AsHadh2, ketoreductase from A. suum with high selectivity of branched substrates; EcTesB, thioesterase from E. coli for generating free hydroxyacids; RePhaA, thiolase from R. eutropha; RePhaB, ketoreductase from R. eutropha; RePhaC, PHA polymerase from $R$. eutropha. HB, 3-hydroxybutyric acid; HV, hydroxyvaleric acid; HMB, 3hydroxy-2-methylbutyric acid; HMV, 3-hydroxy-2-methylvaleric acid).

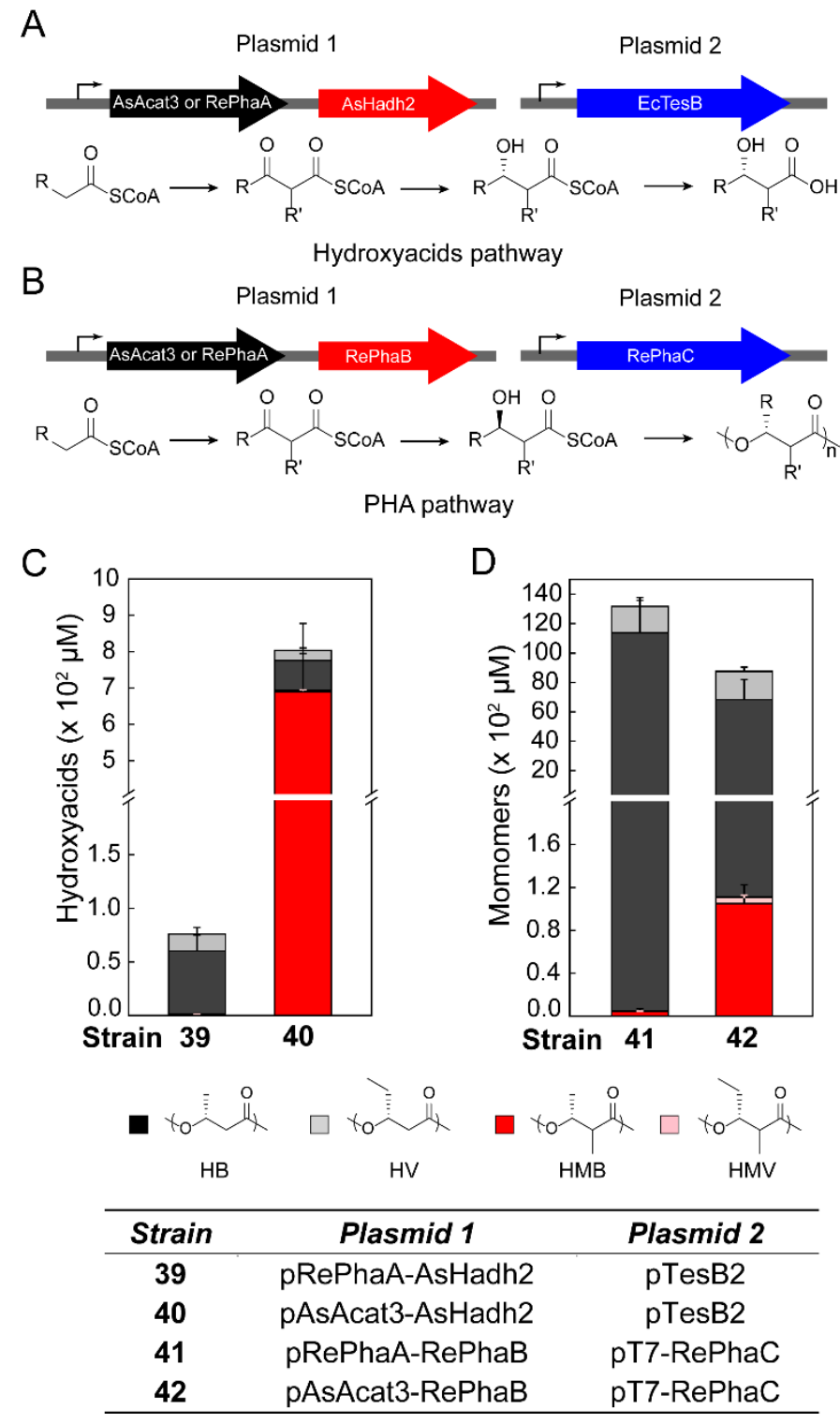


Figure S2. PHA gene cluster in Candidatus Accumulibacter phophatis clade IIA str. UW1. Diagram of the PHA cluster found in the activated sludge metagenome (GenBank No. CP001715.1).

\begin{tabular}{lll} 
& CapPhaC1 CapPhaBo & CapPhaA \\
\hline Gene & Locus_tag & Annotation \\
\hline CapPhaC1 & CAP2UW1_3191 & poly- $\beta$-hydroxybutyrate polymerase domain protein \\
CapPhaB0 & CAP2UW1_3190 & short-chain dehydrogenase/reductase \\
CapPhaA & CAP2UW1_3189 & acetyl-CoA acetyltransferase \\
CapMaoC1 & CAP2UW1_3188 & MaoC domain protein dehydratase \\
CapMaoC2 & CAP2UW1_3187 & MaoC domain protein dehydratase \\
CapPhaE & CAP2UW1_3186 & putative poly(3-hydroxyalkanoate) synthase component \\
CapPhaC & CAP2UW1_3185 & poly(R)-hydroxyalkanoic acid synthase, class III, PhaC subunit \\
\hline
\end{tabular}


Figure S3. Characterization of PHA polymerases RePhaC and CapPhaEC. (A) Protein purification. RePhaC purification: lane 1, pre-induction; lane 2, post-induction; lane 3, soluble lysate; lane 4, insoluble lysate; lane 5, Ni-NTA purified protein $\mathrm{His}_{6} \mathrm{~N}-\mathrm{RePhaC}$. CapPhaEC purification: lane 6, Ni-NTA purified proteins of CapPhaE and CapPhaC-His 6 C; lane 7, NiNTA purified protein of CapPhaE-His 6 C. (B), (C) and (D) Steady-state kinetic data for $3 R$ hydroxyacyl-CoA polymerization by $\mathrm{RePhaC}$ and CapPhaEC. The data were fit to the Hill Michaelis-Menten equation $v_{0}=v_{\max }[\mathrm{S}]^{\mathrm{n}} /\left(K_{\mathrm{M}}^{\mathrm{n}}+[\mathrm{S}]^{\mathrm{n}}\right)$. The amounts of enzyme used to obtain each curve are summarized in the Materials and Methods. Error bars represent mean \pm standard error $(\mathrm{n}=3)$. (E) Comparison of kinetic parameters of RePhaC and CapPhaEC for unbranched (3R-hydroxybutyryl-CoA) and branched (3R-hydroxy-2-methylbutyryl-CoA) substrates. nd, not detectable.
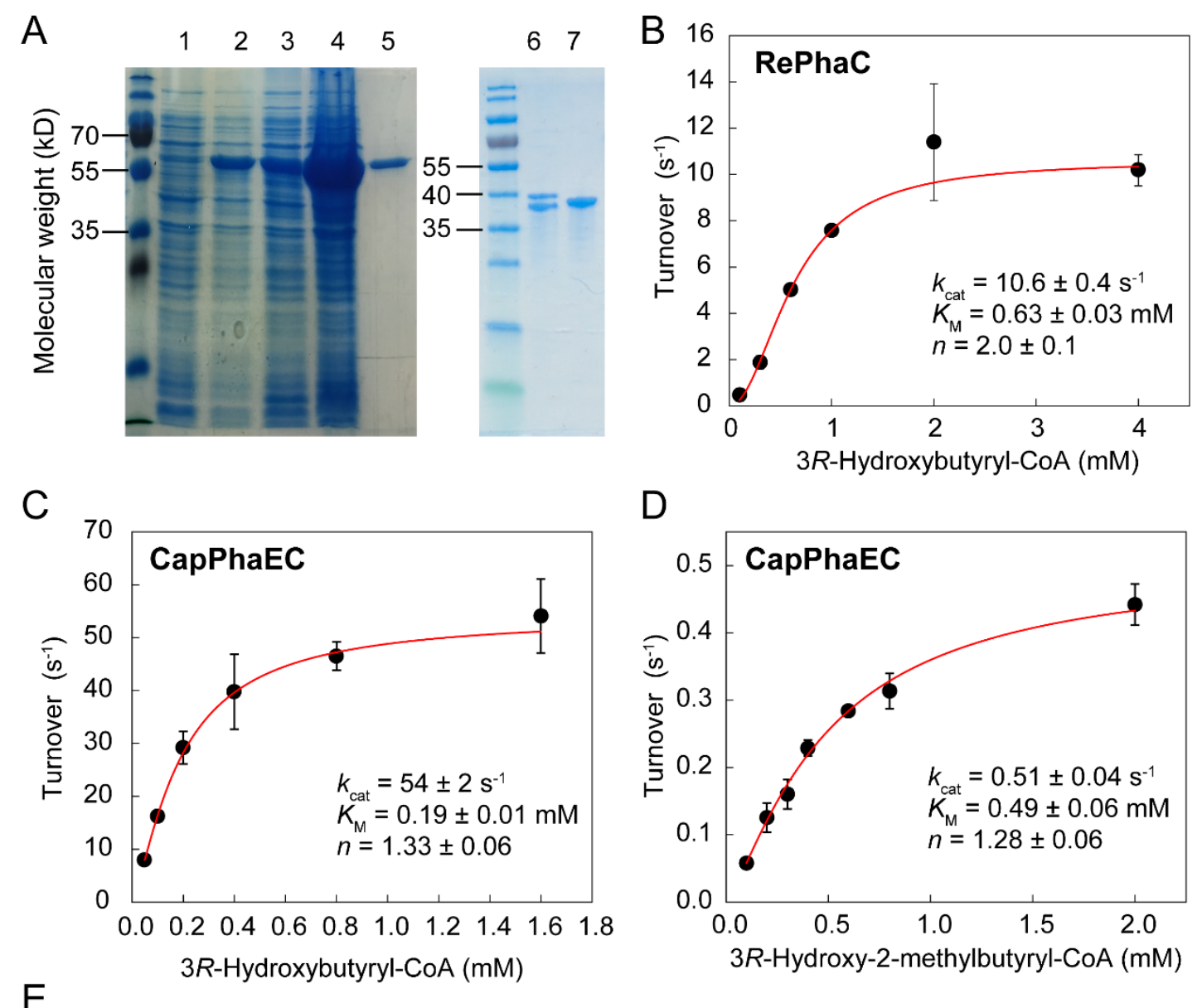

D

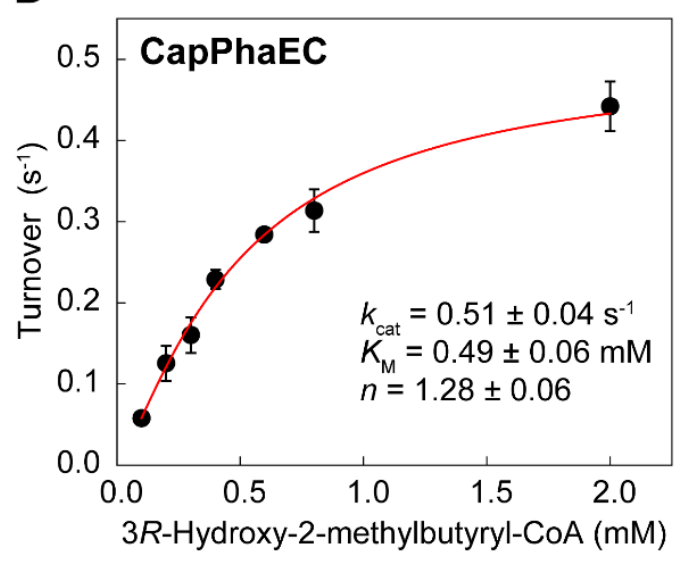

\begin{tabular}{llccc}
\hline Enzyme & Substrate & $\boldsymbol{k}_{\text {cat }}\left(\mathbf{s}^{-1}\right)$ & $\boldsymbol{K}_{\mathrm{M}}(\mathbf{m M})$ & $\boldsymbol{k}_{\text {cat }} / \boldsymbol{K}_{\mathbf{M}}\left(\mathbf{M}^{-1} \mathbf{s}^{-1}\right)$ \\
\hline \multirow{2}{*}{ RePhaC } & $3 R$-Hydroxybutyryl-CoA & $10.6 \pm 0.4$ & $0.63 \pm 0.03$ & $(1.7 \pm 0.1) \times 10^{4}$ \\
& $3 R$-Hydroxy-2-methylbutyryl-CoA & nd & nd & - \\
\hline \multirow{2}{*}{ CapPhaEC } & $3 R$-Hydroxybutyryl-CoA & $54 \pm 2$ & $0.19 \pm 0.01$ & $(2.9 \pm 0.2) \times 10^{5}$ \\
& 3R-Hydroxy-2-methylbutyryl-CoA & $0.51 \pm 0.04$ & $0.49 \pm 0.06$ & $(1.0 \pm 0.2) \times 10^{3}$ \\
\hline
\end{tabular}


Figure S4. Synthesis of 3-oxo-2-methylbutyryl-CoA and 3R-hydroxy-2-methylbutyrylCoA. (A) Chemoenzymatic synthesis of 3-oxo-2-methylbutyryl-CoA (OMB-CoA) and $3 R$ hydroxy-2-methylbutyryl-CoA (HMB-CoA). (B) Monitoring of CoA exchange with OMBCoA by HPLC-MS. Black is $m / z=768$ corresponding to CoA, red is $m / z=866$ corresponding to OMB-CoA. (C) Ketoreduction of OMB-CoA to generate $3 R$-hydroxy-2-methylbutyryl-CoA (HMB-CoA) by RePhaB enzyme. Grey is $m / z=746$ corresponding to NADPH, purple is $m / z=$ 744 corresponding to NADP + , blue is $m / z=868$ corresponding to HMB-CoA. (D) Mass spectra of synthesized 3-oxo-2-methylbutyryl-CoA (OMB-CoA) and 3R-hydroxy-2-methylbutyrylCoA (HMB-CoA) substrates.

A
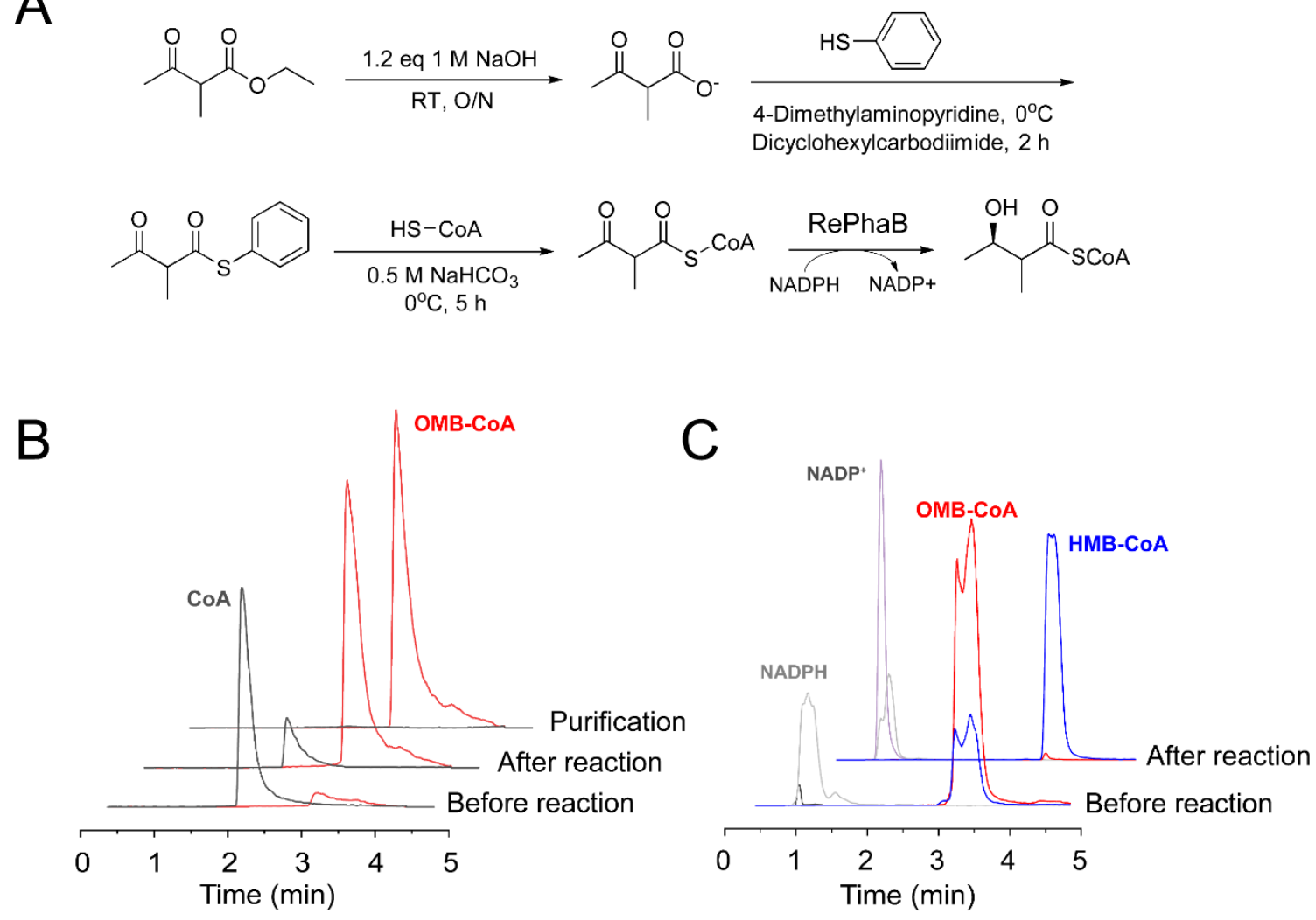

D
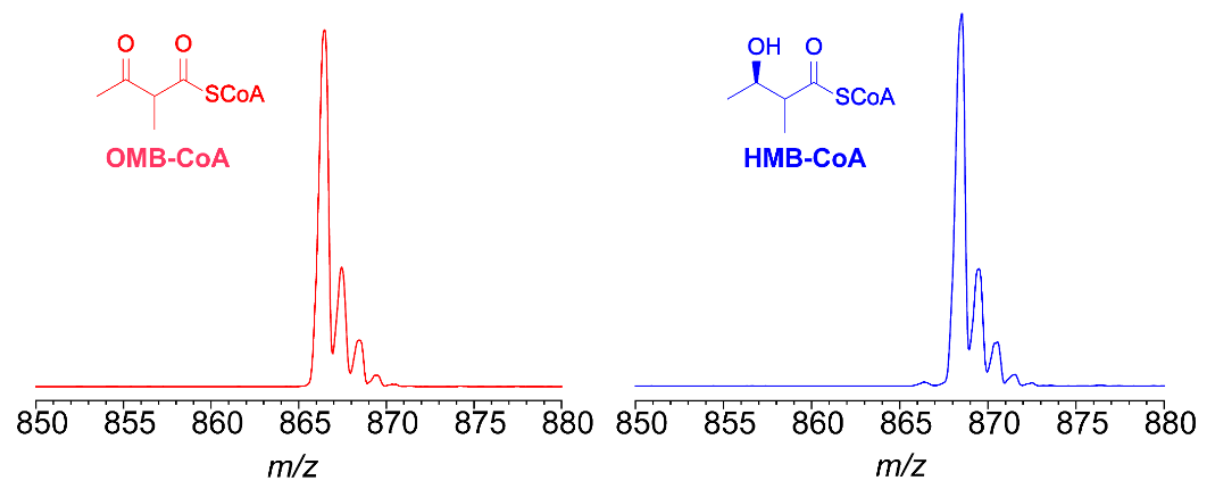
Figure S5. Characterization of the CapPhaB0 ketoreductase. (A) SDS-PAGE of $\mathrm{His}_{10} \mathrm{~N}-$ CapPhaB0: lane 1, pre-induction; lane 2, post-induction; lane 3, soluble lysate; lane 4, insoluble lysate; lane 5, Ni-NTA purified protein. (B) Assay for examining the stereochemical preference of CapPhaB0. (C) Steady-state kinetic data for $3 R$ or $3 S$-hydroxyabutyryl-CoA by CapPhaB0. The data were fitted by Michaelis-Menten equation. The amounts of enzyme used to obtain each curve are summarized in the Materials and Methods. Error bars represent mean \pm standard error $(\mathrm{n}=3)$. (D) Comparison of kinetic parameters of RePhaCapPhaB0 for $3 S$-hydroxybutyryl-CoA and $3 R$-hydroxybutyryl-CoA substrates produced in situ.

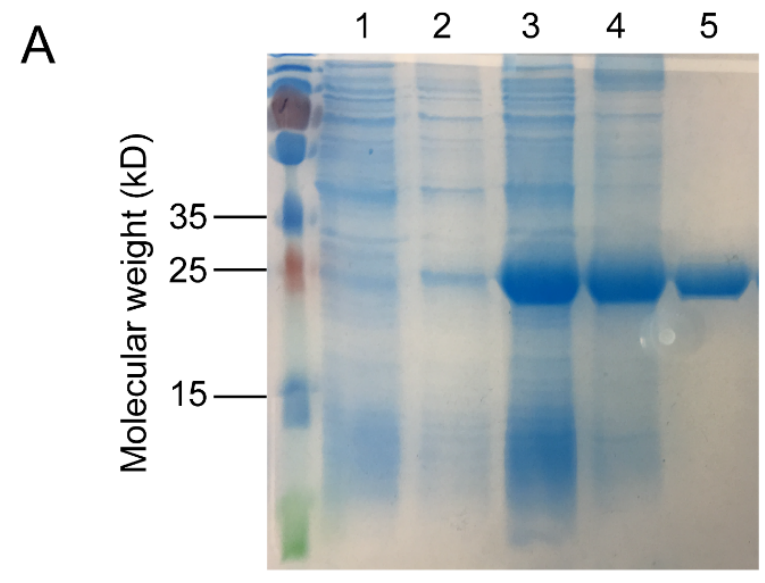

B

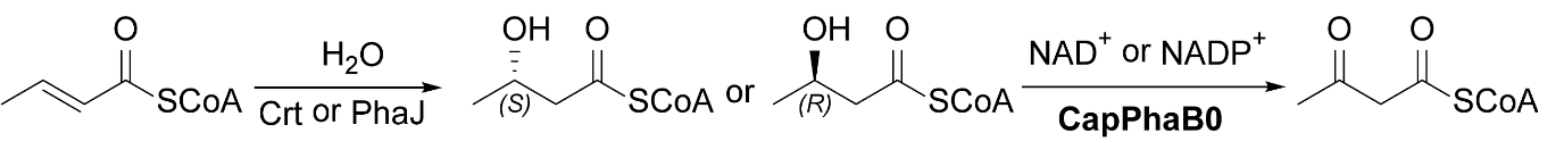

C
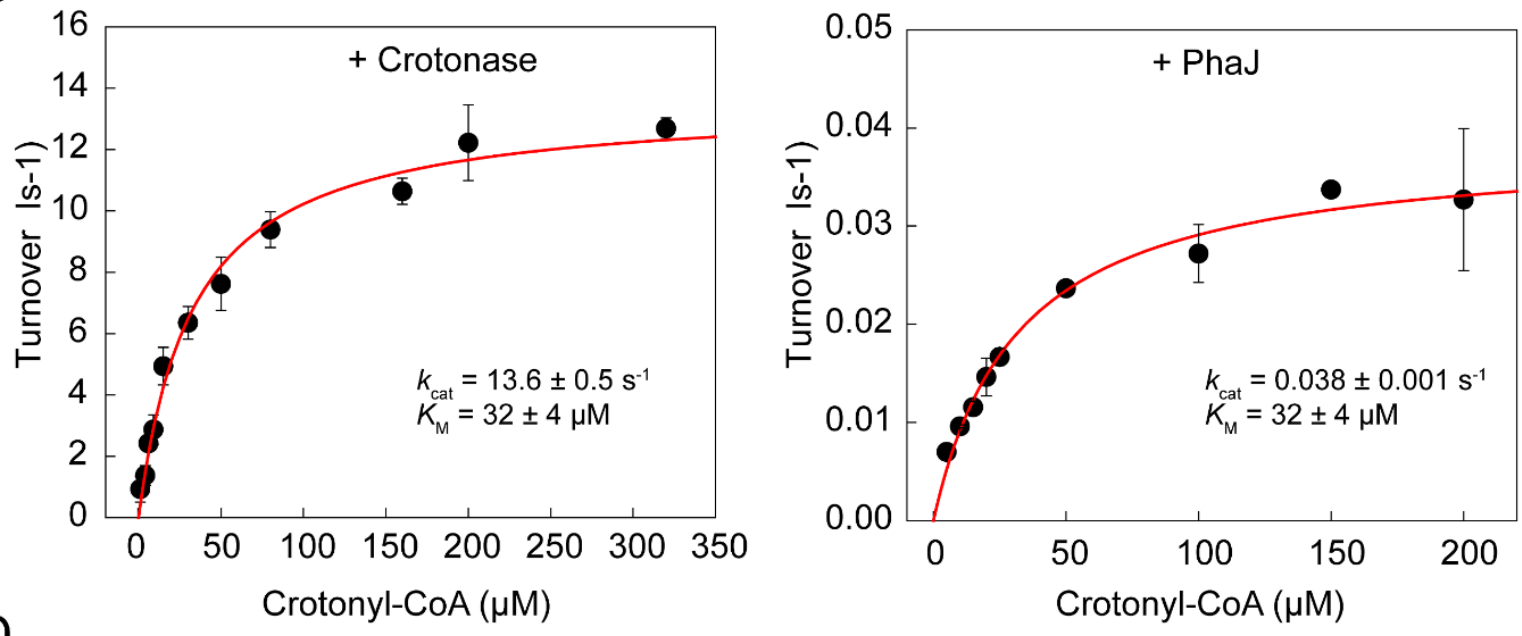

$\mathrm{D}$

\begin{tabular}{|c|c|c|c|}
\hline Substrate & $k_{\text {cat }}\left(\mathbf{s}^{-1}\right)$ & $K_{M}(\mu \mathrm{M})$ & $k_{\text {cat }} / K_{M}\left(M^{-1} s^{-1}\right)$ \\
\hline 3S-hydroxybutyryl-CoA (Crt-NAD+) & $13.6 \pm 0.5$ & $34 \pm 4$ & $(4.1 \pm 0.5) \times 10^{5}$ \\
\hline 3R-hydroxybutyryl-CoA (PhaJ-NAD ${ }^{+}$) & $0.038 \pm 0.001$ & $32 \pm 4$ & $(1.2 \pm 0.1) \times 10^{3}$ \\
\hline 3R-hydroxybutyryl-CoA (PhaJ-NADP ${ }^{+}$) & nd & nd & - \\
\hline
\end{tabular}


Figure S6. CapMaoC1/2 for PHA production. Production of PHA polyesters were tested in $E$. coli BAP1 strains bearing the three-plasmid systems detailed below to compare the ability of CapMaoC1/2's stereochemistry conversion. Strains were cultured for $2 \mathrm{~d}$ at $30{ }^{\circ} \mathrm{C}$ in TB broth containing $2 \%(w / v)$ glucose, $0.2 \%(w / v)$ sodium propionate, and $0.418 \%(w / v)$ MOPS. (A) Pathway for PHA production. (B) The PHA production by MaoC1/2 strain and its control strains. CaHbd: $3 S$-hydroxybutyryl-CoA dehydrogenase gene $h b d$ from Clostridium acetobutylicum.

A
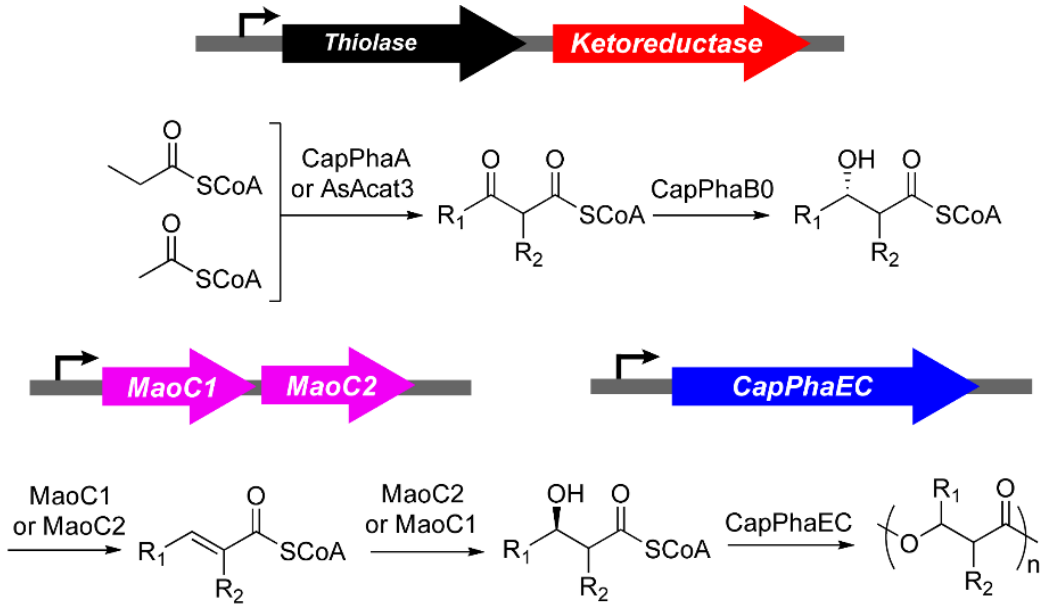

B

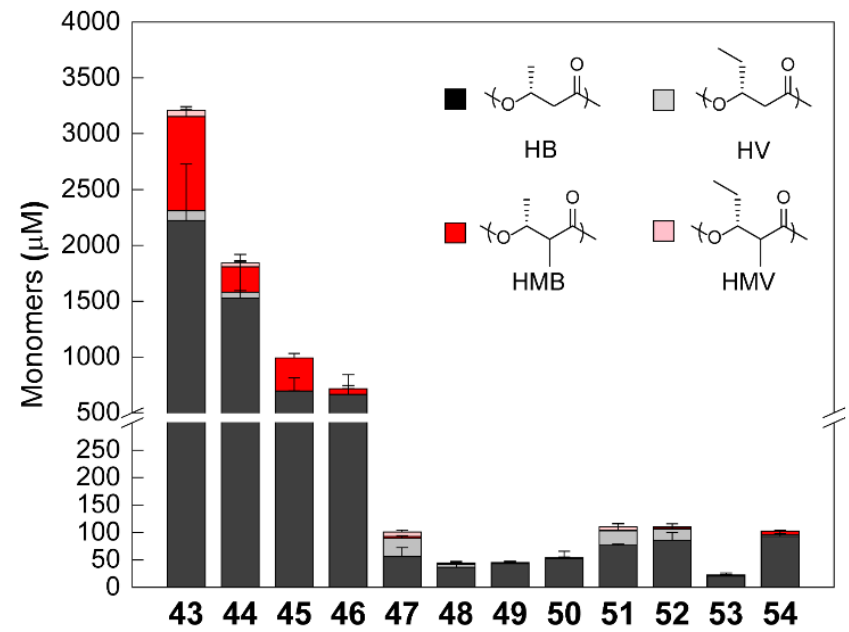

\begin{tabular}{clll}
\hline Strain & \multicolumn{1}{c}{ Plasmid 1 } & \multicolumn{1}{c}{ Plasmid 2 } & \multicolumn{1}{c}{ Plasmid 3 } \\
\hline $\mathbf{4 3}$ & pAsAcat3-RePhaB & pT7-CapPhaEC & pRSFDuet-1 \\
$\mathbf{4 4}$ & pAcat3-RePhaB & pT7-CapPhaEC & pRSF-CapMaoC1/2 \\
$\mathbf{4 5}$ & pRePhaA-RePhaB & pT7-CapPhaEC & pRSFDuet-1 \\
$\mathbf{4 6}$ & pRePhaA-RePhaB & pT7-CapPhaEC & pRSF-CapMaoC1/2 \\
$\mathbf{4 7}$ & pAsAcat3-Hadh2 & pT7-CapPhaEC & pRSFDuet-1 \\
$\mathbf{4 8}$ & pAsAcat3-Hadh2 & pT7-CapPhaEC & pRSF-CapMaoC1/2 \\
$\mathbf{4 9}$ & pAsAcat3-CaHbd & pT7-CapPhaEC & pRSFDuet-1 \\
$\mathbf{5 0}$ & pAsAcat3-CaHbd & pT7-CapPhaEC & pRSF-CapMaoC1/2 \\
$\mathbf{5 1}$ & pAsAcat3-CapPhaB0 & pT7-CapPhaEC & pRSFDuet-1 \\
$\mathbf{5 2}$ & pAsAcat3-CapPhaB0 & pT7-CapPhaEC & pRSF-CapMaoC1/2 \\
$\mathbf{5 3}$ & pCapPhaB0-CapPhaA & pT7-CapPhaEC & pRSFDuet-1 \\
$\mathbf{5 4}$ & pCapPhaB0-CapPhaA & pT7-CapPhaEC & pRSF-CapMaoC1/2 \\
\hline
\end{tabular}


Figure S7. Characterization of the RePhaB and RePhaB Q150C. (A) SDS-PAGE of purified enzymes His ${ }_{10} \mathrm{~N}-\mathrm{RePhaB}$ and His $10 \mathrm{~N}-\mathrm{RePhaB} \_$Q150C: lane 1, His $10-\mathrm{RePhaB}$; lane 2, His $10^{-}$ RePhaB Q150C. (B) Steady-state kinetic data for 3-oxobutyryl-CoA or 3-oxo-2-methylbutyrylCoA by wild type RePhaB or its mutant RePhaB Q150C. The data were fit to the Hill-substrate inhibition equation. The value of the Hill coefficient $n$ was set to 2 for all data fitting here for comparison. The amounts of enzyme used to obtain each curve were summarized in the Materials and Methods. Error bars represent mean \pm standard error $(n=3)$. (C) Comparison of ketoreductase RePhaB and its mutant RePhaB_Q150C for unbranched (3-oxobutyryl-CoA) and branched (3-oxo-2-methylbutyryl-CoA) substrates. (D) Mechanistic scheme for Hill substrate inhibition. E represents RePhaB or its mutant RePhaB_Q150C, A represents NADPH, B represents 3-oxoacyl-CoA, $\mathrm{A}^{\prime}$ represents $\mathrm{NADP}^{+}$, and $\mathrm{B}^{\prime}$ represents product 3-hydroxyacylCoA. $K_{B}, K_{c a t}$, and $K_{I, B}$ are the dissociation constant for each binding step. The $n$ presents the Hill coefficient standing for the cooperativity of substrate binding to the enzyme. (E) Derivation of the modified Hill-substrate inhibition equation.
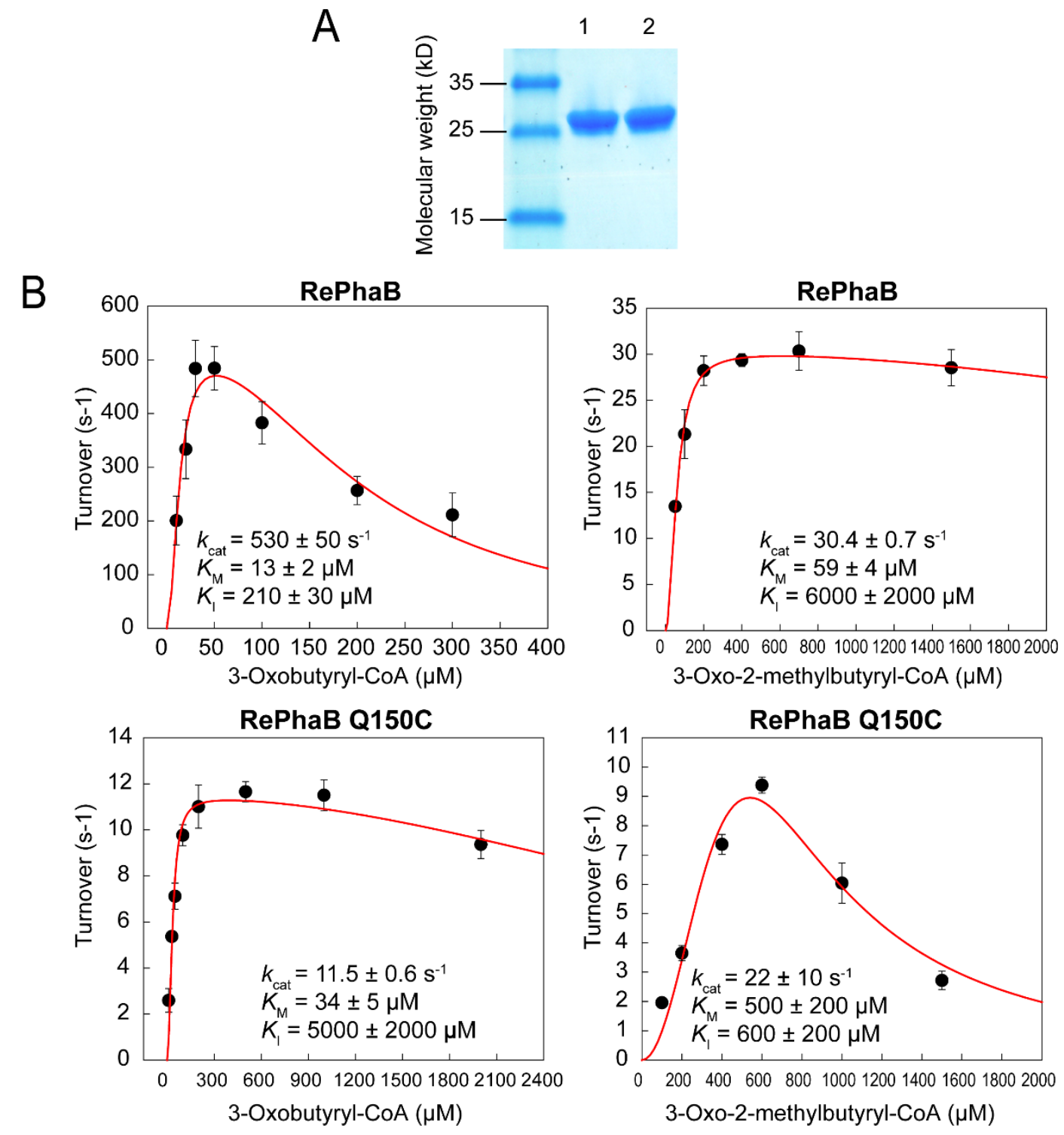


\begin{tabular}{ccccccc}
\hline Enzyme & Substrate & $\boldsymbol{k}_{\mathrm{cat}}\left(\mathbf{s}^{-1}\right)$ & $\boldsymbol{K}_{\mathrm{M}}(\boldsymbol{\mu} \mathrm{M})$ & $\boldsymbol{K}_{\mathrm{l}}(\boldsymbol{\mu} \mathrm{M})$ & $\boldsymbol{k}_{\mathrm{cat}} / \boldsymbol{K}_{\mathrm{M}}\left(\mathrm{M}^{-1} \mathbf{s}^{-1}\right)$ & Relative $\boldsymbol{k}_{\mathrm{cat}} / \boldsymbol{K}_{\mathrm{M}}$ \\
\hline \multirow{2}{*}{ WT } & 3-Oxobutyryl-CoA & $530 \pm 50$ & $13 \pm 2$ & $210 \pm 30$ & $(4.1 \pm 0.8) \times 10^{7}$ & 1 \\
\cline { 2 - 7 } & 3-Oxo-2-methylbutyryl-CoA & $30.4 \pm 0.7$ & $59 \pm 4$ & $6000 \pm 2000$ & $(5.1 \pm 0.3) \times 10^{5}$ & $0.013 \pm 0.003$ \\
\hline \multirow{2}{*}{ Q150C } & 3-Oxobutyryl-CoA & $11.5 \pm 0.6$ & $34 \pm 5$ & $5000 \pm 2000$ & $(3.4 \pm 0.5) \times 10^{5}$ & 1 \\
\cline { 2 - 7 } & 3-Oxo-2-methylbutyryl-CoA & $20 \pm 10$ & $500 \pm 200$ & $600 \pm 200$ & $(5 \pm 3) \times 10^{4}$ & $0.14 \pm 0.09$ \\
\hline
\end{tabular}

D

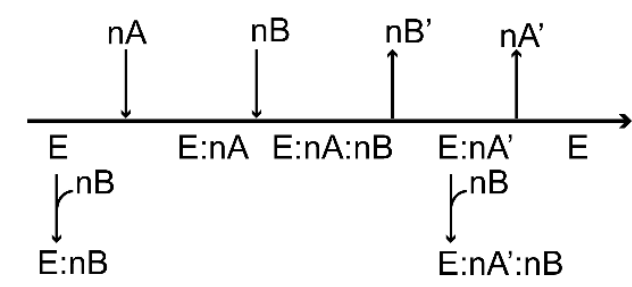

Simplification

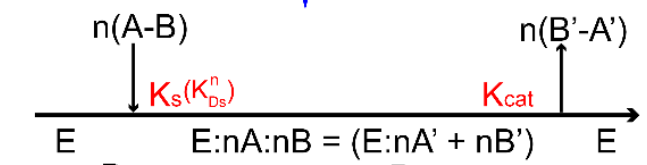

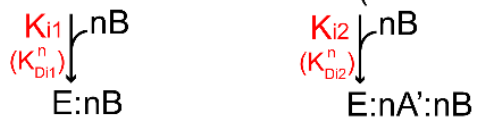

E

Modified Hill-substrate inhibition equation: $v_{0}=\frac{V_{\max }[B]_{0}^{n}}{K_{M}^{n}+[B]_{0}^{n}\left(1+\frac{[B]_{0}^{n}}{K_{I}^{n}}\right)}$

\section{Experimental conditions:}

$[E]_{0},[B]_{0},[A-B]_{0}$ : total amount of each; $v_{0}$ : inital velocity.

$[E]_{0} \leq[B]_{0} ;[A-B]_{0}=[B]_{0}$

Conservation equations:

$[E]_{0}=[E]+[E: n A: n B]+\left[E: n A^{\prime}: n B\right]$

$[A-B]_{0}=[B]_{0}=[B]+[E: n A: n B]+\left[E: n A^{\prime}: n B\right]=[B]$

$[E: n A: n B]=\left[E: n A^{\prime}\right]$ 
Statement of rate dependence:

$$
v_{0}=K_{c a t}\left[E: n A^{\prime}: n B^{\prime}\right]
$$

\section{Definition of binding constants:}

$$
\begin{aligned}
& K_{B}=\frac{[E][A-B]^{n}}{[E: n A: n B]} ; \frac{[E]}{[E: n A: n B]}=\frac{K_{B}}{[A-B]^{n}}=\frac{K_{B}}{[B]^{n}}=\frac{K_{B}}{[B]_{0}^{n}} ; K_{B}=K_{M}^{n} \\
& K_{I, B}=\frac{\left[E: n A^{\prime}\right][B]^{n}}{\left[E: n A^{\prime}: n B\right]} ; \frac{\left[E: n A^{\prime}\right]}{\left[E: n A^{\prime}: n B\right]}=\frac{[E: n A: n B]}{\left[E: n A^{\prime}: n B\right]}=\frac{K_{I, B}}{[B]^{n}}=\frac{K_{I, B}}{[B]_{0}^{n}} ; K_{I, B}=K_{I}^{n}
\end{aligned}
$$

Derivation:

$$
\begin{aligned}
& \frac{v_{0}}{[E]_{0}}=\frac{K_{c a t}[E: n A: n B]}{[E]+[E: n A: n B]+\left[E: n A^{\prime}: n B\right]} \\
& \frac{v_{0}}{[E]_{0}}=\frac{K_{c a t}}{\frac{[E]}{[E: n A: n B]}+1+\frac{\left[E: n A^{\prime}: n B\right]}{\left[E: n A^{\prime}: n B^{\prime}\right]}} \\
& \frac{v_{0}}{[E]_{0}}=\frac{K_{c a t}}{\frac{K_{B}}{[B]_{0}^{n}}+1+\frac{[E]}{[E: n A: n B]}+\frac{[B]_{0}^{n}}{K_{I, B}}} \\
& \frac{v_{0}}{[E]_{0}}=\frac{K_{c a t}}{\frac{K_{S}}{[B]_{0}^{n}}+1+\frac{[B]_{0}^{n}}{K_{I, B}}} \\
& \frac{v_{0}}{[E]_{0}}=\frac{K_{c a t}}{\frac{K_{M}^{n}}{[B]_{0}^{n}}+1+\frac{[B]_{0}^{n}}{K_{I}^{n}}} \\
& \frac{v_{0}}{[E]_{0}}=\frac{K_{c a t}[B]_{0}^{n}}{K_{M}^{n}+[B]_{0}^{n}+\frac{[B]_{0}^{2 n}}{K_{I}^{n}}} \\
& \frac{v_{0}}{[E]_{0}}=\frac{K_{c a t}[B]_{0}^{n}}{K_{M}^{n}+[B]_{0}^{n}\left(1+\frac{[B]_{0}^{n}}{K_{I}^{n}}\right)} \\
& v_{0}=\frac{[E]_{0} K_{c a t}[B]_{0}^{n}}{K_{M}^{n}+[B]_{0}^{n}\left(1+\frac{[B]_{0}^{n}}{K_{I}^{n}}\right)} \\
& v_{0}=\frac{V_{\max }[B]_{0}^{n}}{K_{M}^{n}+[B]_{0}^{n}\left(1+\frac{[B]_{0}^{n}}{K_{I}^{n}}\right)}
\end{aligned}
$$


Table S2. Check of the RePhaB library quality. 115 mutants from the RePhaB libraries were sequenced to provide preliminary information about its diversity.

\begin{tabular}{ccc}
\hline Mutation No. & Mutation site & Mutated/Total \\
\hline 1 & $\mathrm{I} 15$ & $9 / 10$ \\
\hline 2 & $\mathrm{~T} 92$ & $5 / 5$ \\
\hline 3 & $\mathrm{D} 94$ & $5 / 5$ \\
\hline 4 & $\mathrm{~S} 140$ & $4 / 5$ \\
\hline 5 & $\mathrm{~V} 141$ & $5 / 5$ \\
\hline 6 & $\mathrm{~N} 142$ & $5 / 5$ \\
\hline 7 & $\mathrm{Q} 147$ & $5 / 5$ \\
\hline 8 & $\mathrm{~F} 148$ & $5 / 5$ \\
\hline 9 & $\mathrm{Q} 150$ & $5 / 5$ \\
\hline 10 & $\mathrm{Y} 153$ & $3 / 5$ \\
\hline 11 & $\mathrm{P} 183$ & $4 / 5$ \\
\hline 12 & $\mathrm{G} 184$ & $4 / 5$ \\
\hline 13 & $\mathrm{Y} 185$ & $5 / 5$ \\
\hline 14 & $\mathrm{I} 186$ & $4 / 5$ \\
\hline 15 & $\mathrm{~T} 188$ & $5 / 5$ \\
\hline 16 & $\mathrm{M} 190$ & $3 / 5$ \\
\hline 17 & $\mathrm{~V} 191$ & $4 / 5$ \\
\hline 18 & $\mathrm{I} 194$ & $5 / 5$ \\
\hline 19 & $\mathrm{R} 195$ & $5 / 5$ \\
\hline 20 & $\mathrm{~V} 198$ & $5 / 5$ \\
\hline 21 & $\mathrm{I} 202$ & $5 / 5$ \\
\hline 22 & $\mathrm{~L} 243$ & $4 / 5$
\end{tabular}


Figure S8. High throughput screening of RePhaB library by polyester fermentation. The fresh colonies were inoculated using $3 \mathrm{~mm}$ glass beads into the 24-well plates, which were then covered with gas permeable film. The production was carried out aerobically for $2 \mathrm{~d}$. The resulted cells were hydrolyzed by $\mathrm{KOH}$, naturized by acids, and filtered to be samples for triple quadrupole LCMS detection.

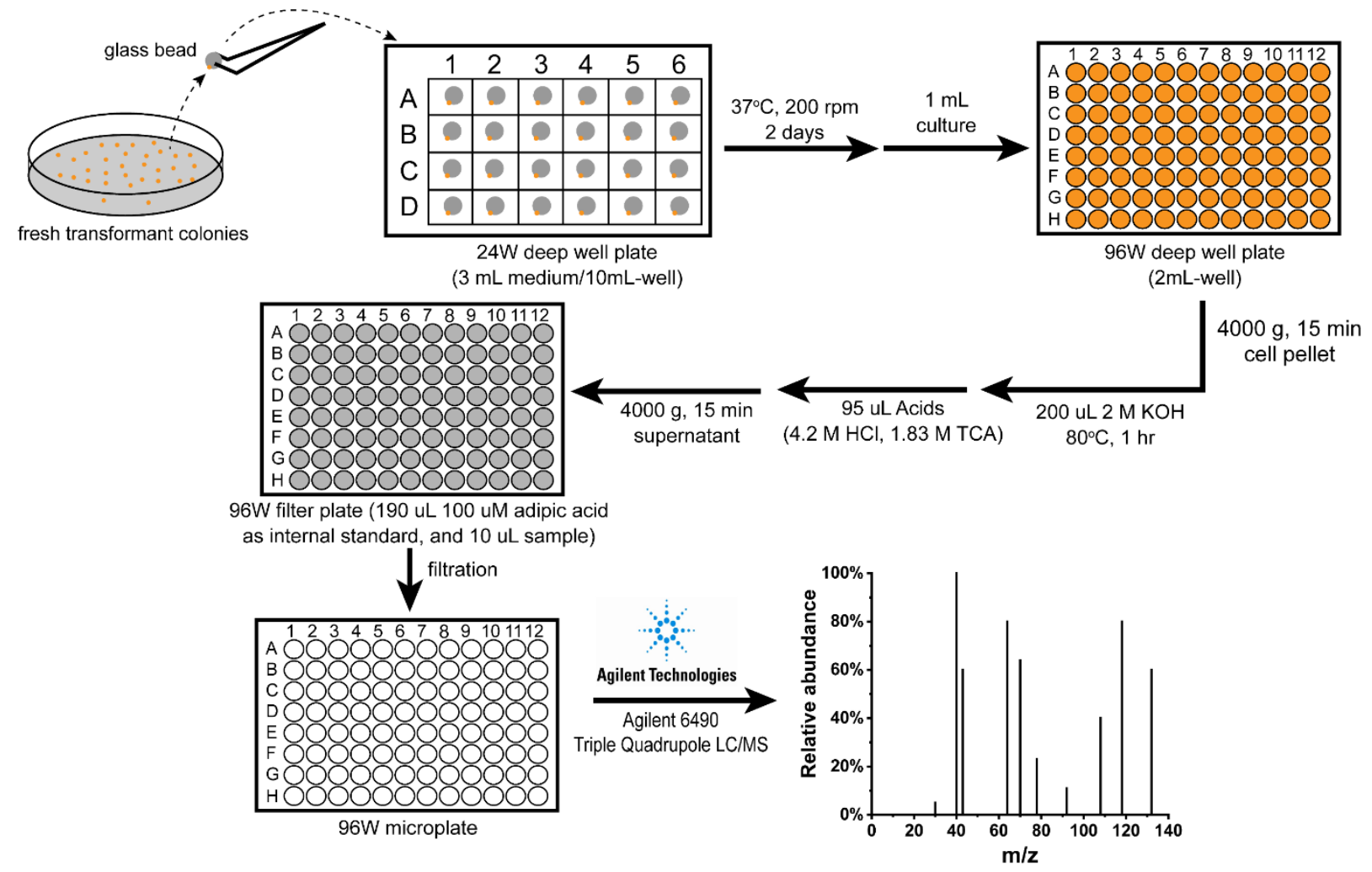


Figure S9. Quantification of hydroxyacids by LC/Triple Quadrupole MS (QQQ). (A) Standard curves of various hydroxyacids generated by LC/QQQ. Peak areas were normalized using adipic acid as an internal standard. (B) Analysis of the monomer composition of PHA samples by LC/QQQ. The [M-H] ${ }^{-}$MRM transitions used for HB, HV, HMB, HMV and adipic acid were $m / z 103.1 \rightarrow 59.2,117.1 \rightarrow 59.2,117.1 \rightarrow 73.2,131.1 \rightarrow 73.2,145.1 \rightarrow 83.1$, respectively. HB, 3-hydroxybutyric acid; HV, 3-hydroxyvaleric acid; HMB, 3-hydroxy-2-methylbutryic acid; HMV, 3-hydroxy-2-methylvaleric acid.
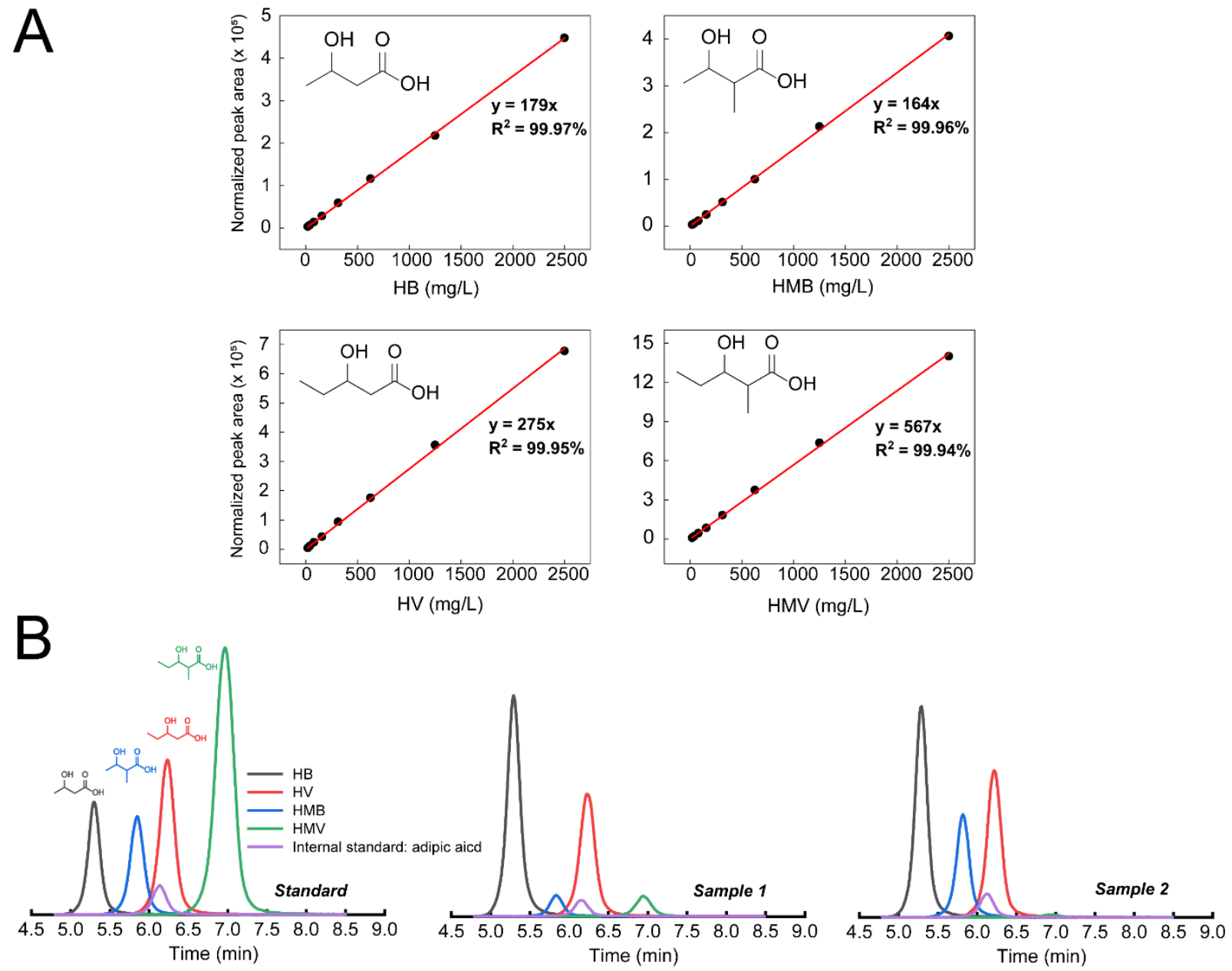
Figure S10. RePhaB pocket library screening. (A) Distribution of RePhaB mutants according to the titers of monomers. (B) The radar chart shows the general relative production of each monomer in polyesters by RePhaB library. The wild type titer of each monomer is set as 1 . The two top producers are highlighted in red and blue.

A
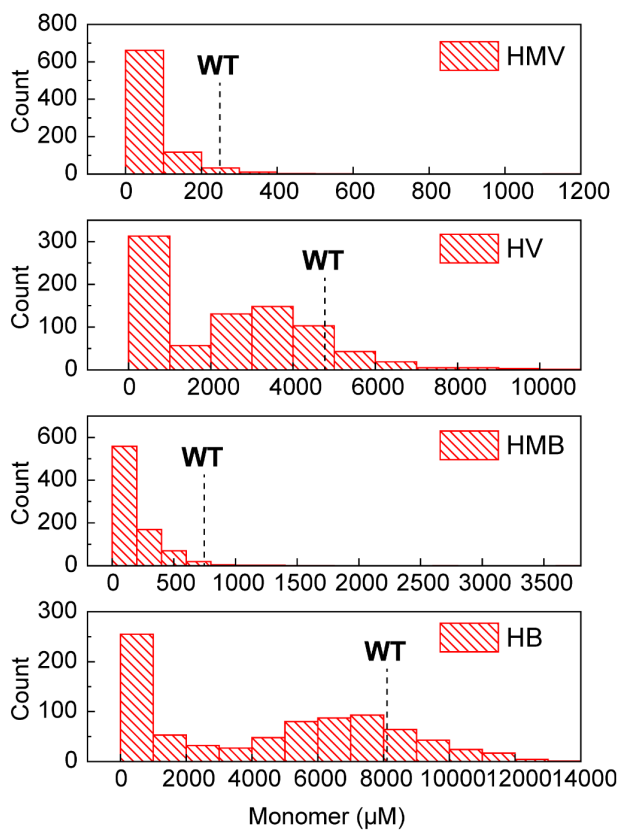

B

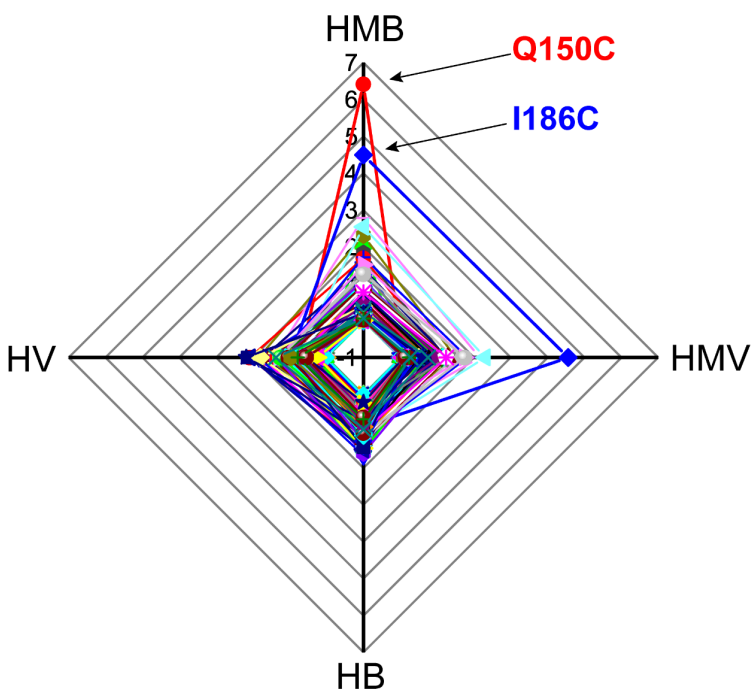


Figure S11. Polyester production by the RePhaB Q150 and I186 mutants. (A) The monomer composition of PHA polymers produced by RePhaB Q150C, RePhaB I186C, and RePhaB Q150C I186C normalized to wt RePhaB. (B) The monomer composition of PHA polymers produced by saturation mutagenesis at position Q150 and I186 normalized to $\mathrm{wt}$ RePhaB. (C) The monomer composition of PHA polymers produced by saturation mutagenesis at position Q150 of RePhaB I186C normalized to RePhaB I186C. (E) The monomer composition of PHA polymers produced by saturation mutagenesis at position I186 of RePhaB Q150C normalized to RePhaB Q150C. Data are mean \pm s.d. of biological replicates $(n=3)$.
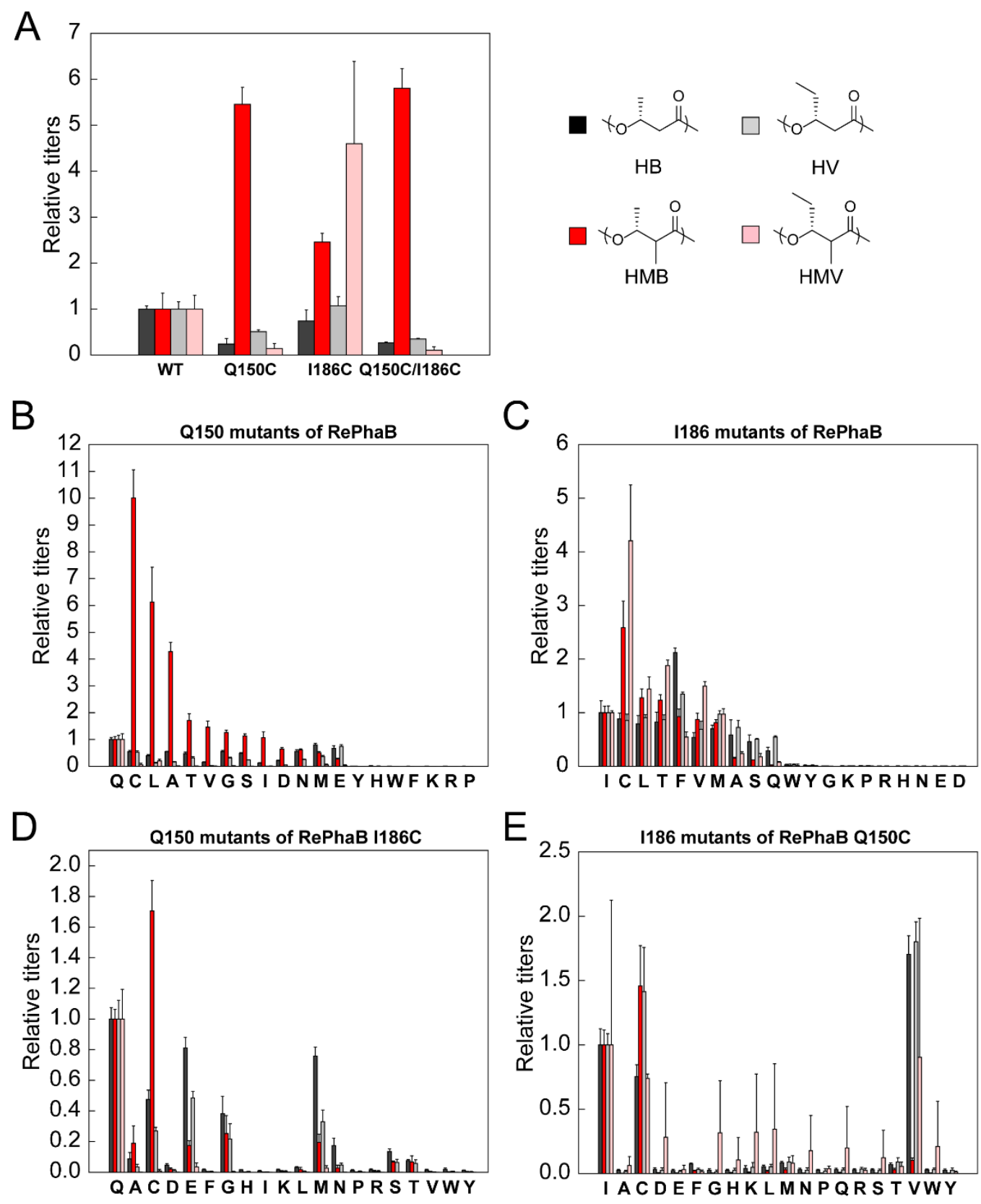
Table S3. Monomer compositions of PHA polyester samples produced on large-scale for polymer characterization. E. coli BAP1 strain was used for polyester production. Strains were cultured for $2 \mathrm{~d}$ at $30^{\circ} \mathrm{C}$ in TB broth containing $2 \%(w / v)$ glucose, $0.2 \%$ sodium propionate, and $0.418 \%(w / v)$ MOPS in baffled flasks except for samples 5, 7, and $\mathbf{8}$. The samples 1, 3, 4, 7 were analyzed by NMR, and all samples were tested by SEC, TGA, and DSC for properties characterization.

\begin{tabular}{|c|c|c|c|c|c|c|}
\hline \multirow{2}{*}{ Sample } & \multicolumn{4}{|c|}{ Monomer ratio (mol\%) } & \multicolumn{2}{|r|}{ Strains } \\
\hline & HB & HMB & HV & HMV & No. & Plasmid 1 / Plasmid 2 \\
\hline 1 & $40 \%$ & $22 \%$ & $38 \%$ & $0 \%$ & 38 & pAsAcat3-RePhaB Q150C / pT7-RePhaEC-CpPct) \\
\hline 2 & $49 \%$ & $28 \%$ & $23 \%$ & $0 \%$ & 55 & pAsAcat3-RePhaB Q150L / pT7-RePhaEC-CpPct \\
\hline 3 & $47 \%$ & $10 \%$ & $38 \%$ & $5 \%$ & 37 & pAsAcat3-RePhaB I186C / pT7-RePhaEC-CpPct \\
\hline 4 & $37 \%$ & $0 \%$ & $62 \%$ & $0 \%$ & 56 & pAsAcat3-RePhaB I186Q / pT7-RePhaEC-CpPct \\
\hline $5^{a}$ & $15 \%$ & $32 \%$ & $52 \%$ & $0 \%$ & 38 & pAsAcat3-RePhaB Q150C / pT7-RePhaEC-CpPct \\
\hline 6 & $54 \%$ & $4 \%$ & $40 \%$ & $1 \%$ & 33 & pAsAcat3-RePhaB / pT7-RePhaEC \\
\hline $7^{b}$ & $99 \%$ & $0 \%$ & $1 \%$ & $0 \%$ & 33 & pAsAcat3-RePhaB / pT7-RePhaEC \\
\hline $8^{a}$ & $55 \%$ & $1 \%$ & $44 \%$ & $0 \%$ & 33 & pAsAcat3-RePhaB / pT7-RePhaEC \\
\hline 9 & $100 \%$ & $0 \%$ & $0 \%$ & $0 \%$ & - & Purchased from Sigma-Aldrich \\
\hline
\end{tabular}

a) Produced in 24-deep well plates.

b) No sodium propionate added. 
Figure S12. NMR spectra of PHA polyesters. (A) $1 \mathrm{D}{ }^{1} \mathrm{H}$ and ${ }^{13} \mathrm{C}$ NMR spectra of a PHB homopolymer produced by strain 33 (sample 7). $1 \% \mathrm{HV}$ was ignored. (B) $1 \mathrm{D}^{1} \mathrm{H}$ and ${ }^{13} \mathrm{C} N M R$ spectra of the poly(HB-co-HV) polyester produced by strain 56 (sample 4). (C) $1 \mathrm{D}^{1} \mathrm{H}$ and ${ }^{13} \mathrm{C}$ NMR spectra of the poly (HB-co-HV-co-HMB) polyester produced by strain 38 (sample 1). (D) $1 \mathrm{D}^{1} \mathrm{H}$ and ${ }^{13} \mathrm{C}$ NMR spectra of the poly (HB-co-HV-co-HMB-co-HMV) polyester produced by strain 37 (sample 3). (E) (F) (G) (H) 2D HSQC NMR of $\alpha$-branched polyester samples 7, 4, 1, and 2, respectively. HB: 3-hydroxybutyrate, HV: 3-hydroxyvalerate, HMB: 3-hydroxy-2methylbutyrate, HMV: 3-hydroxy-2-methylvalerate.

A
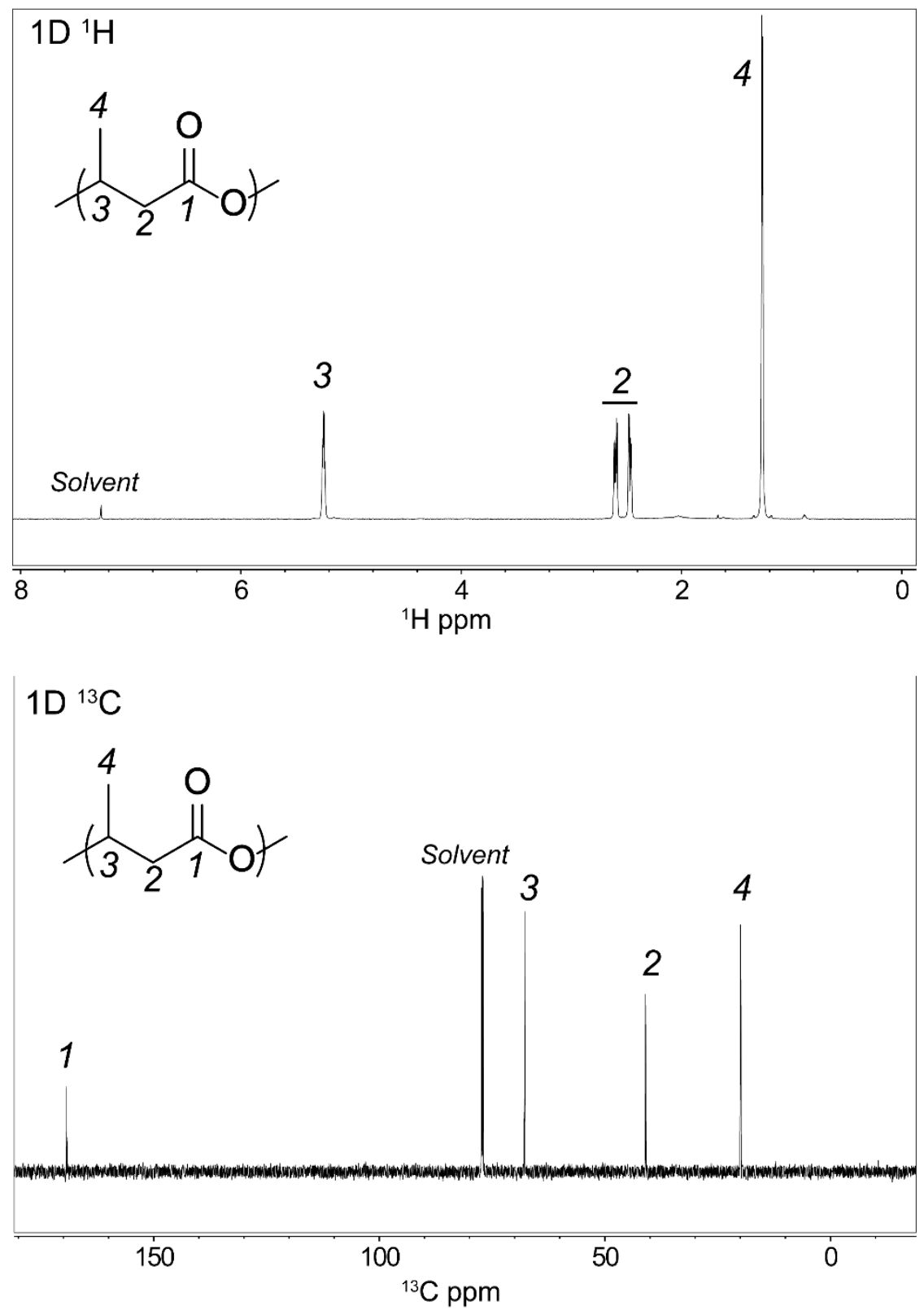
B
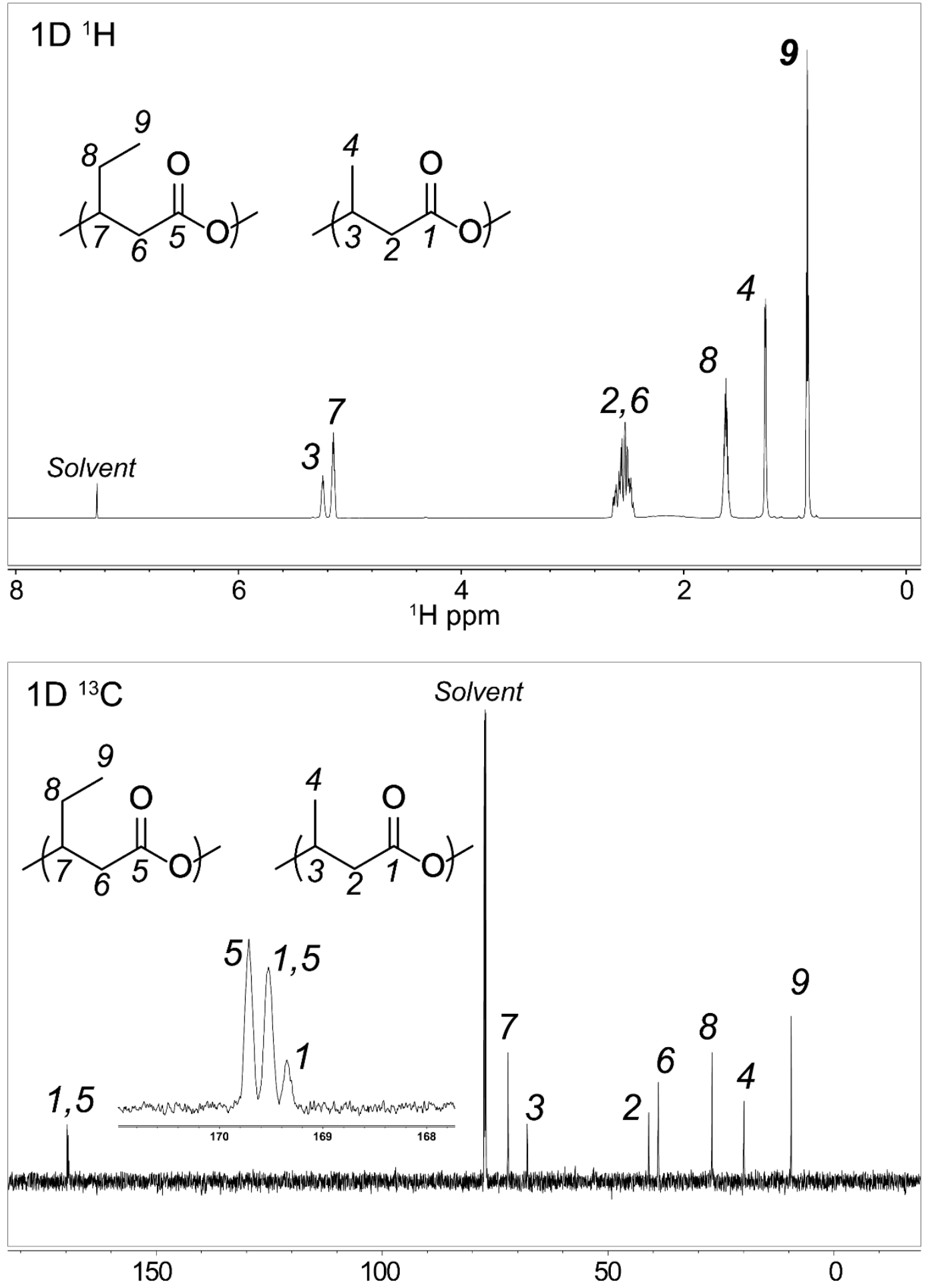

50

${ }^{13} \mathrm{C} \mathrm{ppm}$ 

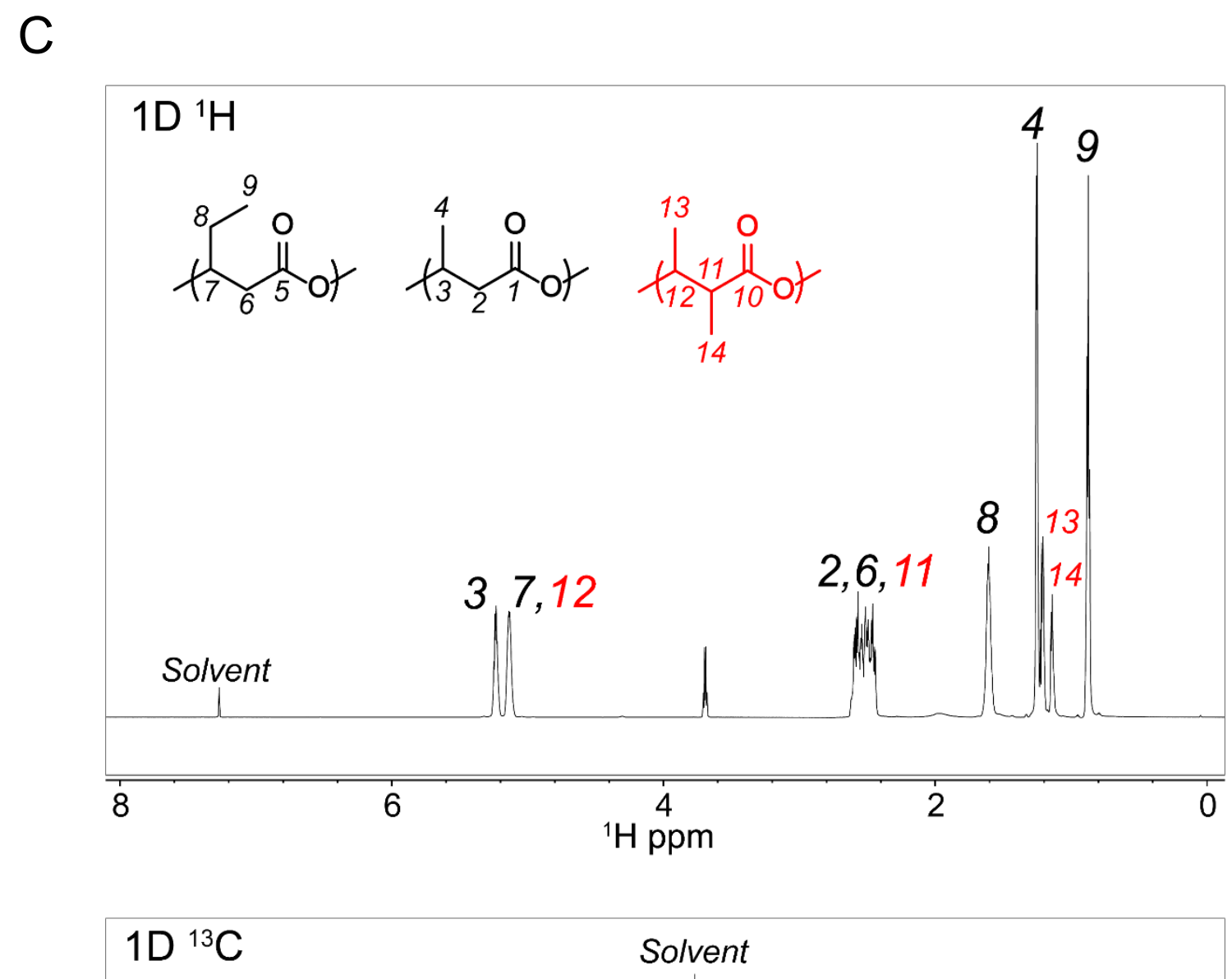

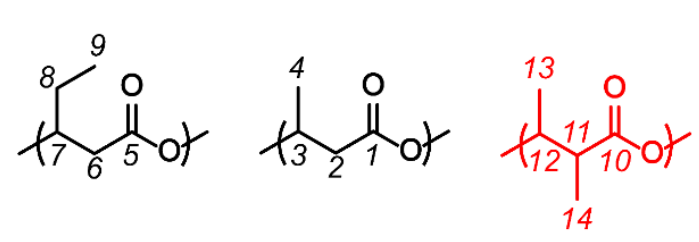
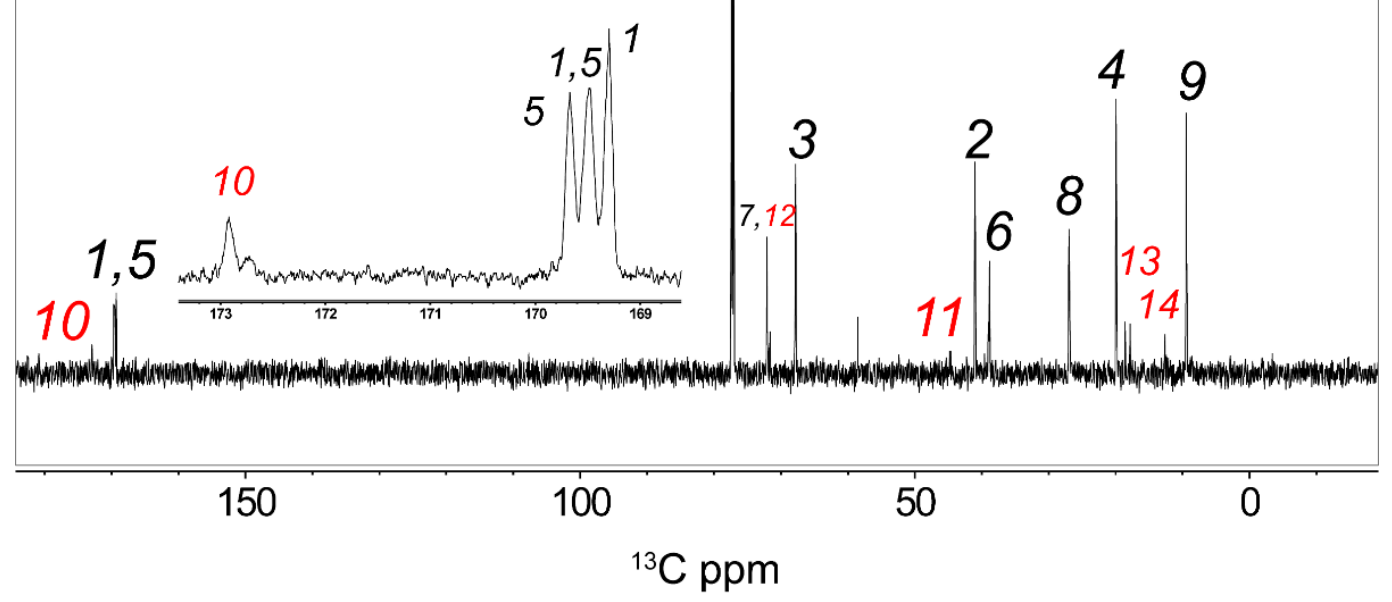
D
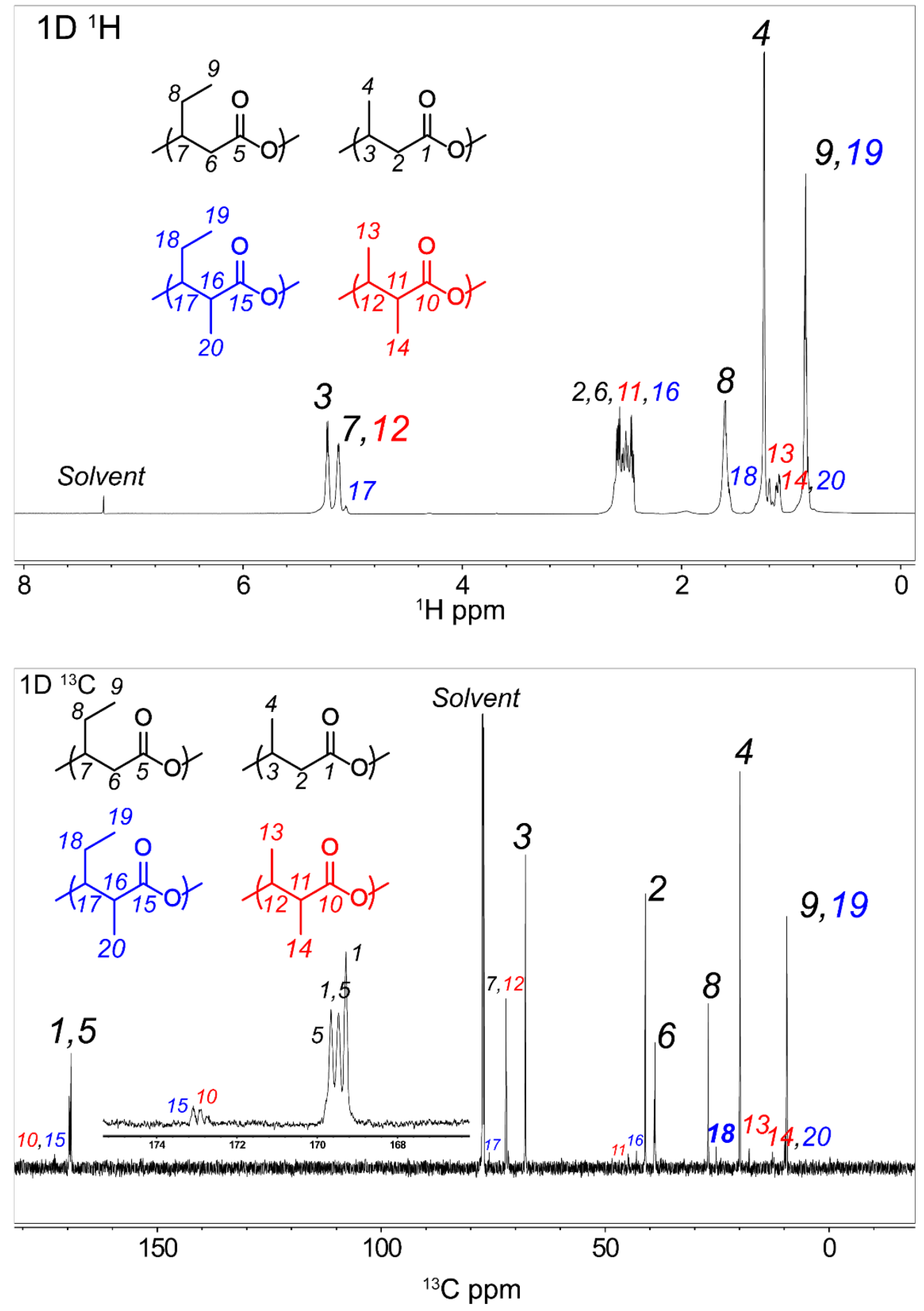
E

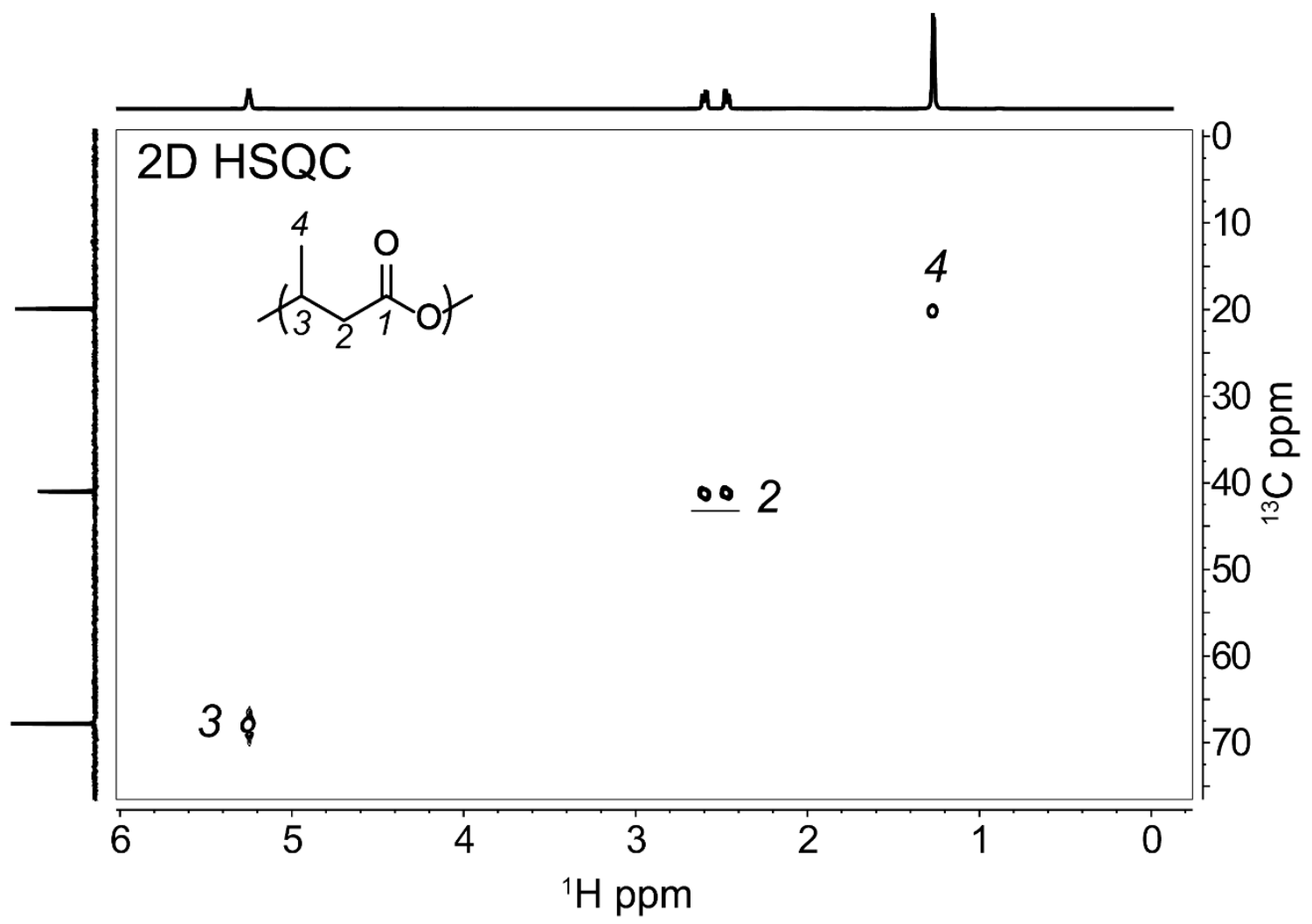


F

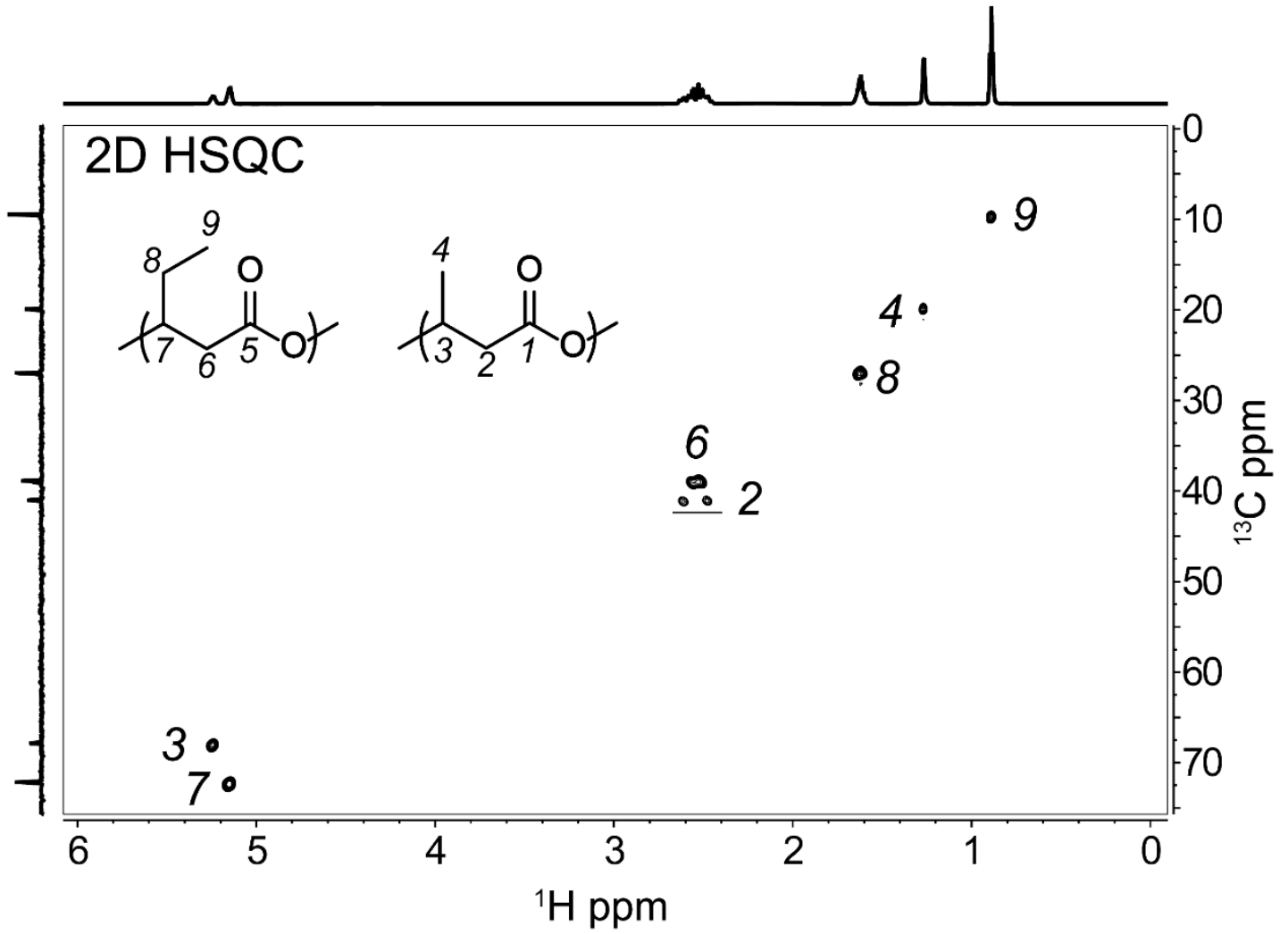




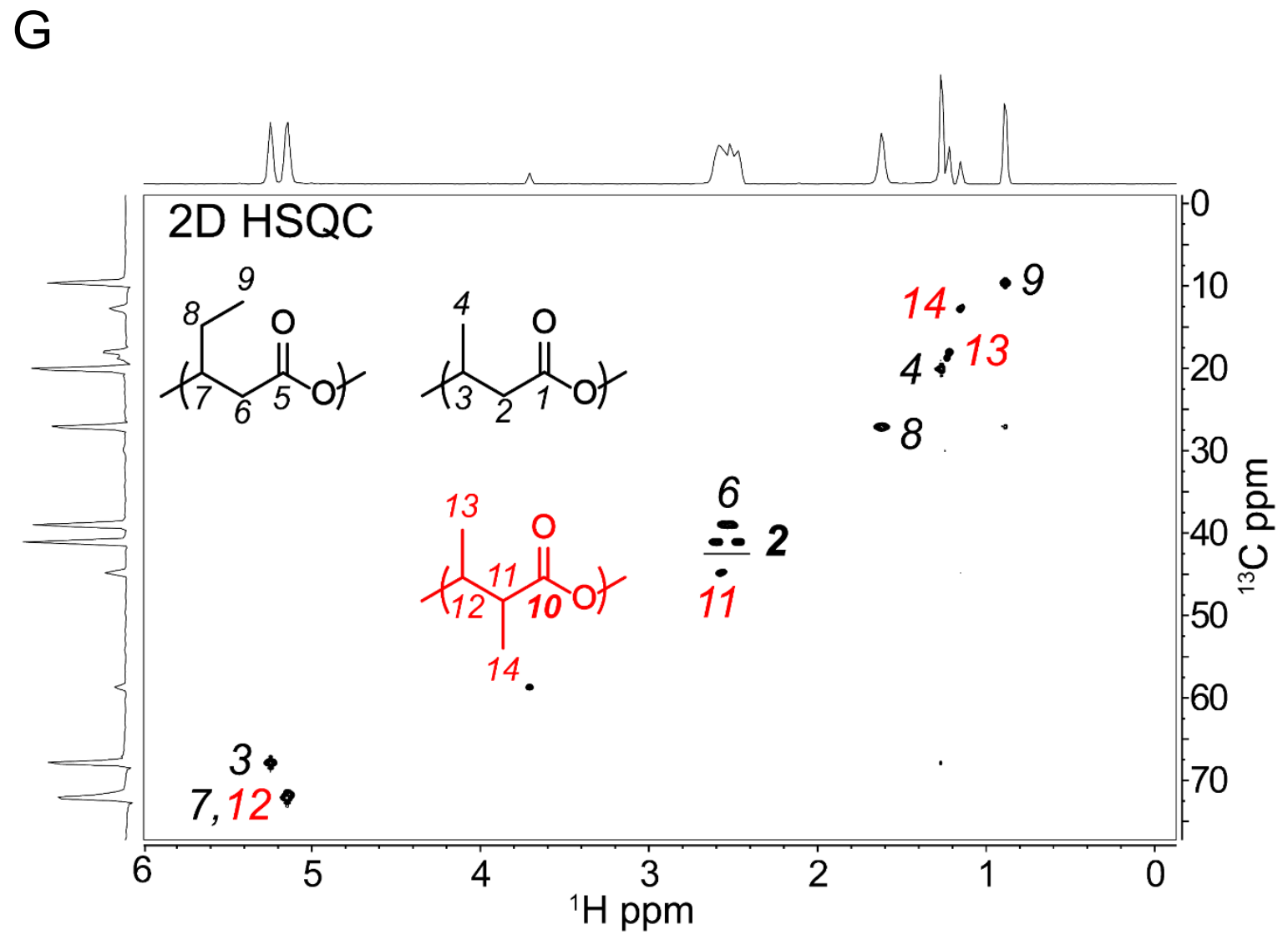


$\mathrm{H}$

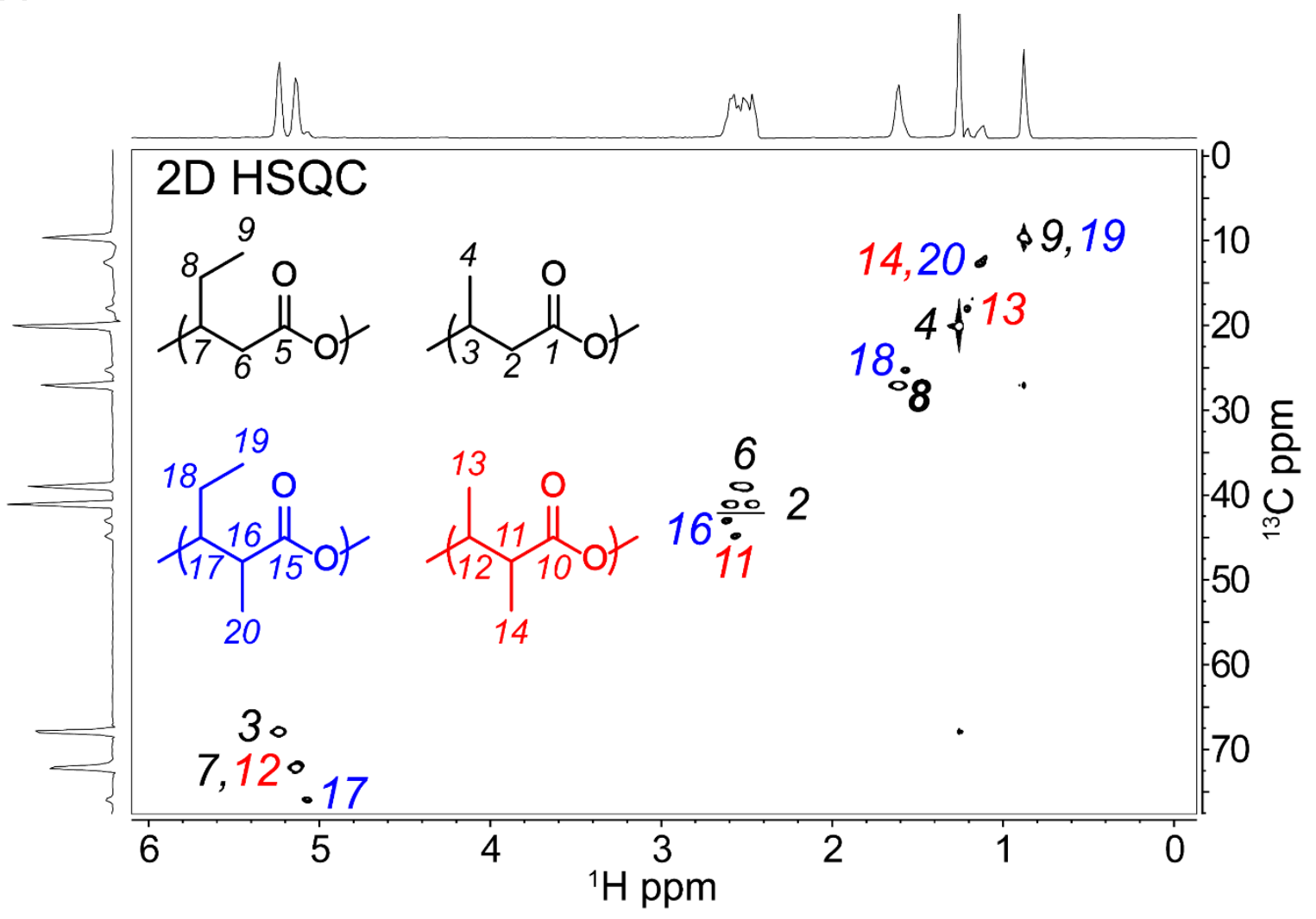


Figure S13. Chloroform size-exclusion chromatography (SEC) analysis of PHA polyesters. The number-average molar mass $\left(M_{\mathrm{n}}\right)$, weight-average molar mass $\left(M_{\mathrm{w}}\right)$, and dispersity (Đ) for the PHA polyesters were determined using chloroform $\left(\mathrm{CHCl}_{3}\right) \mathrm{SEC}$, which uses polystyrene (PS) standards for molar mass determination. (A) $\mathrm{CHCl}_{3} \mathrm{SEC}$ traces of samples where sample concentration is 3-4 mg/mL. (B) Values for $M_{\mathrm{n}}$ and $M_{\mathrm{w}}$, and $Đ$. The $M_{\mathrm{n}}$ values obtained for the samples using $\mathrm{CHCl}_{3} \mathrm{SEC}$ range from $80-500 \mathrm{kDa}$ with dispersities between 1.1 and 2.5. The samples with the highest mol\% PHB, the PHB homopolymer (Sample 9) and PHA with $99 \mathrm{~mol} \%$ PHB (Sample 7), display the highest $M_{\mathrm{n}}$ values at 430 and $500 \mathrm{kDa}$ respectively, while also displaying the lowest dispersities at 1.1 and 1.2 respectively. Samples with lower mol\% PHB displayed lower molar masses and higher dispersity values. Additionally, the asymmetry, tailing, and non-gaussian shape of the peaks in the $\mathrm{CHCl}_{3} \mathrm{SEC}$ traces suggests a degree of inhomogeneity in the samples.

A

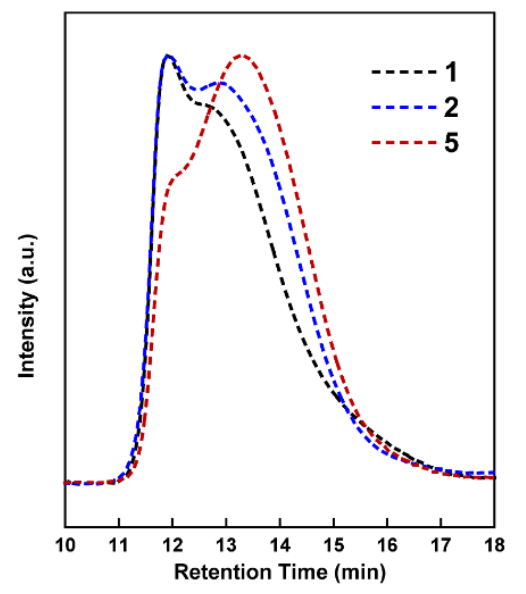

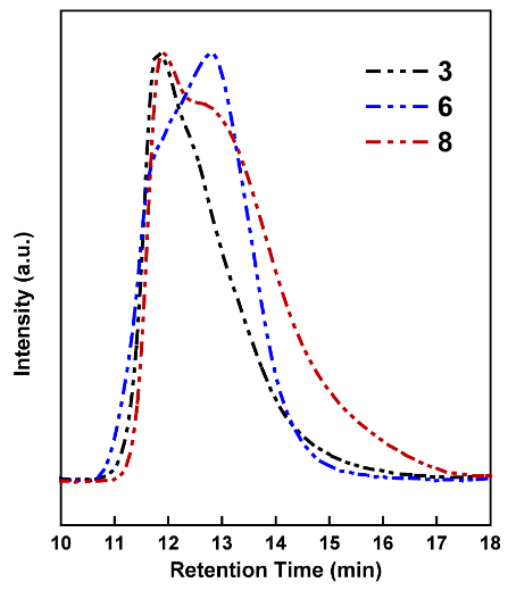

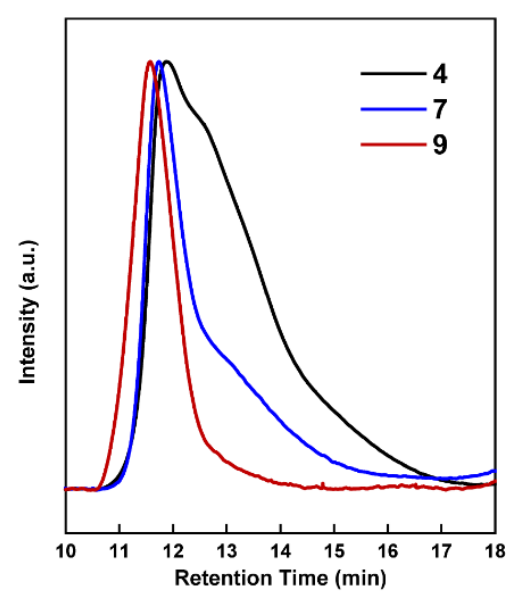

B

\begin{tabular}{cccc}
\hline Sample & $\begin{array}{c}\boldsymbol{M}_{\mathbf{n}} \\
\left(\mathrm{g} \mathrm{mol}^{-1}\right)\end{array}$ & $\begin{array}{c}\boldsymbol{M}_{\mathbf{w}} \\
\left(\mathrm{g} \mathrm{mol}^{-1}\right)\end{array}$ & $\boldsymbol{\Xi}$ \\
\hline 1 & $8.7 \times 10^{4}$ & $1.9 \times 10^{5}$ & 2.2 \\
2 & $9.4 \times 10^{4}$ & $2.0 \times 10^{5}$ & 2.2 \\
3 & $1.7 \times 10^{5}$ & $3.0 \times 10^{5}$ & 1.8 \\
4 & $9.7 \times 10^{4}$ & $2.4 \times 10^{5}$ & 2.5 \\
5 & $8.0 \times 10^{4}$ & $1.7 \times 10^{5}$ & 2.1 \\
6 & $1.7 \times 10^{5}$ & $2.8 \times 10^{5}$ & 1.7 \\
7 & $5.0 \times 10^{5}$ & $5.8 \times 10^{5}$ & 1.2 \\
8 & $8.8 \times 10^{4}$ & $2.1 \times 10^{5}$ & 2.4 \\
9 & $4.3 \times 10^{5}$ & $4.7 \times 10^{5}$ & 1.1 \\
\hline
\end{tabular}


Figure S14. THF size-exclusion chromatography analysis of PHA polyesters. (A) Samples $\mathbf{1 , 2}, \mathbf{3}, \mathbf{4}, \mathbf{5}, \mathbf{6}$, and $\mathbf{8}$ were analyzed in THF at $1 \mathrm{mg} / \mathrm{L}, 3-4 \mathrm{mg} / \mathrm{L}$, and 6-8 mg/L. The PHB homopolymers (Sample 7 and 9) were insoluble in THF. Values of $M_{\mathrm{n}}$ and $M_{\mathrm{w}}$, and $\mathrm{D}$ for the analyzed samples are summarized in (B). The molar mass values for the PHA polyesters determined using THF SEC were higher than those determined with $\mathrm{CHCl}_{3} \mathrm{SEC}$, displaying $M_{\mathrm{n}}$ values ranging from $120-1300 \mathrm{kDa}$ and dispersities between 1.5 and 1.9. In contrast to the $\mathrm{CHCl}_{3}$ SEC traces, the peak shapes of the THF SEC traces are more symmetric with minimal tailing. The symmetry in the THF SEC traces may be due to the fact that the data plotted in the traces are taken using the refractive index detector or possible interactions between the polymer and solvent that are not present in $\mathrm{CHCl}_{3}$. Additionally, the differences in column type, detector, and solvent between instruments should be considered when analyzing peak shape and makes direct comparison between the resulting SEC traces difficult.

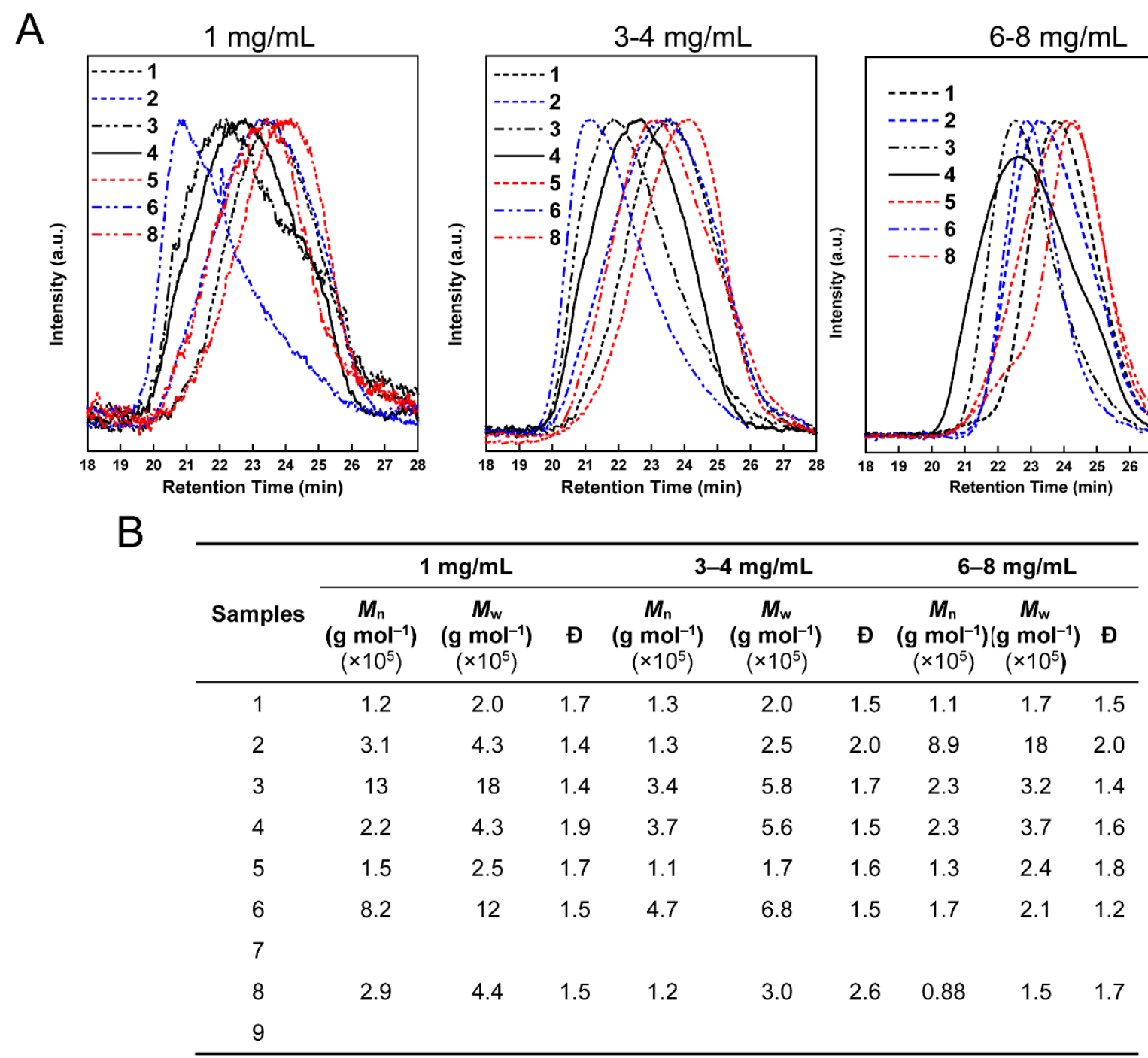


Figure S15. Thermogravimetric analysis (TGA) of PHA polyester. Thermogravimetric analysis was conducted on all samples under a dry $\mathrm{N}_{2}$ atmosphere. (A) TGA traces for polyester samples. The key values are summarized in (B). Thermal decomposition of all samples was rapid, occurring in a single step, which is analogous to previously reported PHB homopolymers. ${ }^{18-19}$ The copolymers displayed $5 \%$ mass loss temperatures $\left(\mathrm{T}_{\mathrm{d}, 5 \%}\right)$ in the range of $241-266{ }^{\circ} \mathrm{C}$ compared to a $\mathrm{T}_{\mathrm{d}, 5 \%}$ value of $227{ }^{\circ} \mathrm{C}$ for the commercial PHB homopolymer (Sample 9).

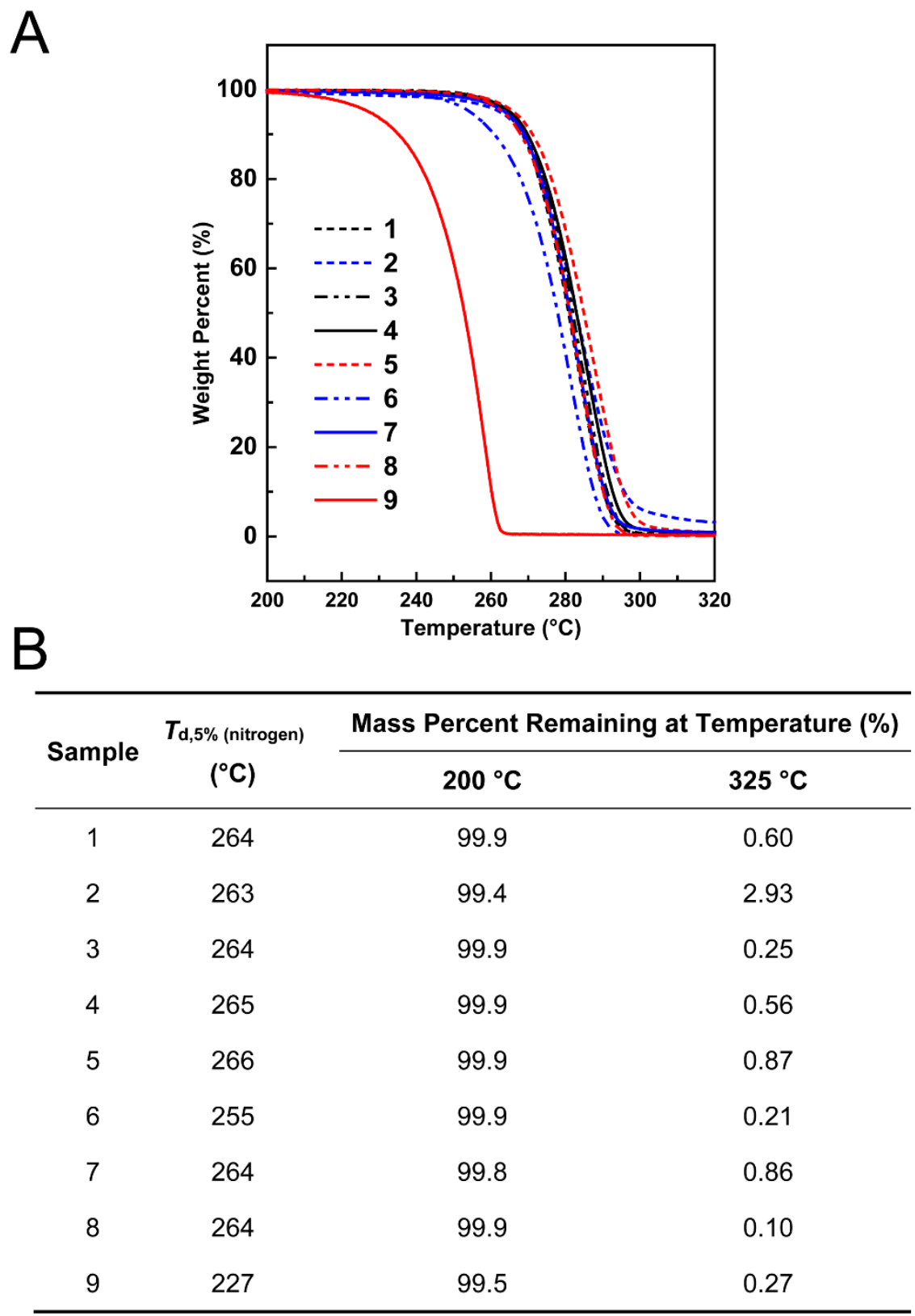


Figure S16. Differential scanning calorimetry (DSC) analysis of PHA polyesters. (A) DSC traces for polyester samples (top) first heating cycle from 50 to $200{ }^{\circ} \mathrm{C}$, (middle) cooling cycle from 200 to $-50{ }^{\circ} \mathrm{C}$, (bottom) second heating cycle from -50 to $200{ }^{\circ} \mathrm{C}$. (B) Values of $T_{\mathrm{g},} T_{\mathrm{m}}, T_{\mathrm{c}}$, $\Delta H_{\mathrm{c}}$, and $\Delta H_{\mathrm{m}}$. The $T_{\mathrm{m}}, T_{\mathrm{g}}$, and percent crystallinity for the PHA polyesters were lower than those in the PHB homopolymer for all copolymer compositions. The thermal properties were affected by the composition of the backbone, particularly the mol\% of HMB or HMV in the polymers. Overall, an increase in the mol\% of HMV or HMB in the PHA polyesters resulted in a decrease in both the melting temperature $\left(T_{\mathrm{m}}\right)$ and the degree of crystallinity. A more detailed description is found below:

- Samples 7, 9 (PHB homopolymers) The polymer with 1 mol\% HV (Sample 7) produced by our strain displayed a similar melting range to the commercial PHB homopolymer (Sample 9) of approximately $130-167^{\circ} \mathrm{C}$. However, the $\mathrm{T}_{g}$ of sample 7 is $-8{ }^{\circ} \mathrm{C}$ in contrast to $0{ }^{\circ} \mathrm{C}$ of sample 9. Additionally, the peak $T_{\mathrm{m}}$ values of the melting endotherm of the PHB homopolymer (Sample 9) are lower than the values that have been reported for polymers of similar molar mass of $170-180{ }^{\circ} \mathrm{C} .{ }^{19}$

- Sample 4 (0\% HMB or HMV, 62\% HV): Interestingly, in contrast to previous studies $^{16,20-21}$, the copolymer (Sample 4) with $62 \mathrm{~mol} \% \mathrm{HV}$ and $0 \mathrm{~mol} \% \mathrm{HMV}$ or HMB displayed no melting transition. Prior reports on $\mathrm{P}(\mathrm{HB}-\mathrm{co}-\mathrm{HV})$ copolymers with similar PHV mol $\%$ of $53,{ }^{21} 55,{ }^{22}$ and $62 \%{ }^{21}$ report $T_{\mathrm{m}}$ values ranging from $60-100{ }^{\circ} \mathrm{C}$.

- Samples 3, 6, 8 (10\% or less HMV or HMB) The copolymers with $10 \%$ or less of HMV or HMB, displayed minimal to no crystallinity with no clear correlation between HMB or HMV content and degree of crystallinity. The sample 3 with the highest HMV content of $5 \mathrm{~mol} \%$ and $10 \mathrm{~mol} \%$ HMB displayed a broad melting transition with a range from $120-160{ }^{\circ} \mathrm{C}$ and a percent crystallinity of approximately $2 \%$, whereas the sample with $1 \% \mathrm{HMV}$ and $4 \% \mathrm{HMB}$ appeared to be completely amorphous. This is somewhat counterintuitive as an increase in heterogeneity of the polymer backbone (through increased HMV content) should decrease effective chain packing and therefore decrease crystallization. The sample 8 with only $1 \% \mathrm{HMB}$ and $0 \% \mathrm{HMV}$ displayed both a cold crystallization peak at $81{ }^{\circ} \mathrm{C}$ and a melting transition from $90-150{ }^{\circ} \mathrm{C}$. These data do not suggest a clear trend between HMV and HMB content and crystallinity in the copolymers. Despite the variations in HMV and HMB content, the three polymers displayed similar $T_{\mathrm{g}}$ values of $-11,-13$, and $-11{ }^{\circ} \mathrm{C}$ in order of increasing mol\% HMV.

The presence of multiple melting endotherms, as seen in the DSC traces of the PHA polyesters, could arise from a variety of factors, including compositional heterogeneity, multiple crystalline forms, or recrystallization during heating in the DSC. The isodimorphism of $3 \mathrm{HB}$ and $3 \mathrm{HV}$ (i.e., similarity in shape and size of the monomers) allows them to be incorporated into the same crystal lattice without distortion of the lattice parameters. ${ }^{22}$ The presence of two melting peaks in the trace of the PHB homopolymer as well as the isodimorphism between the two monomers suggests that the multiple observed melting peaks may in fact be due to recrystallization upon heating. Previous studies into the crystallization behavior of PHB and PHA copolymers suggest that the low temperature melting peak corresponds to crystals formed at $T_{\mathrm{c}}$, whereas the high temperature peak corresponds to material that has undergone recrystallization 
during heating. ${ }^{18}$ This hypothesis can be investigated through isothermal crystallization studies at increasing heating rates to determine if there is a coalescence of the two peaks into one.

- Samples 1, 2, 5 (at least 22\% HMB or HMV) The DSC thermograms of the PHA polyesters with at least $22 \mathrm{~mol} \%$ of HMV or HMB display only a glass transition temperature, indicating that these polymers are amorphous materials with no crystallinity. This is in contrast to previous reports of $\mathrm{P}(\mathrm{HB}-\mathrm{co}-\mathrm{HV}-\mathrm{co}-\mathrm{HMB}-\mathrm{co}-\mathrm{HMV})$ copolymers which displayed melting transitions and low degrees of crystallinity at a variety of HMB and HMV loadings. ${ }^{16,23}$ It should be noted that this deviation from literature precedent may be due to differences in composition between the copolymers. ${ }^{16}$, ${ }^{23}$ The $T_{\mathrm{g}}$ values of all PHA polyesters were well below that of the PHB homopolymer, Sample $9\left(0^{\circ} \mathrm{C}\right)$, ranging from -11 to $-20^{\circ} \mathrm{C}$.
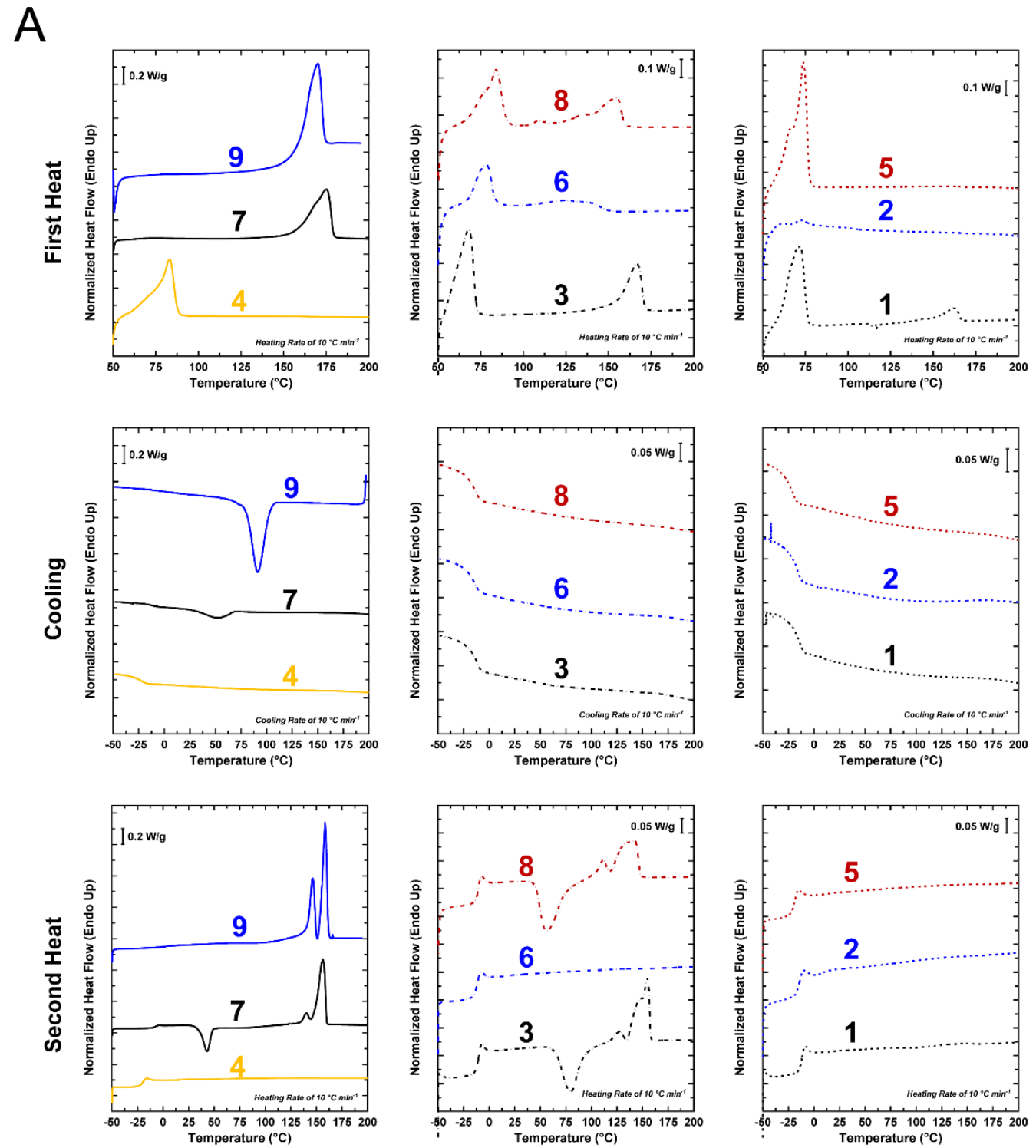


\begin{tabular}{ccccccc}
\hline Sample & $\begin{array}{c}T_{\mathrm{g}} \\
\left({ }^{\circ} \mathrm{C}\right)\end{array}$ & $\begin{array}{c}T_{\mathrm{c}} \\
\left({ }^{\circ} \mathrm{C}\right)\end{array}$ & $\begin{array}{c}\Delta H_{\mathrm{c}} \\
(\mathrm{J} / \mathrm{g})\end{array}$ & $\begin{array}{c}T_{\mathrm{m}, 1} \\
\left({ }^{\circ} \mathrm{C}\right)\end{array}$ & $\begin{array}{c}\Delta H_{\mathrm{m}} \\
(\mathrm{J} / \mathrm{g})\end{array}$ & $\begin{array}{c}\text { "corrected" } \Delta H_{\mathrm{m}} \\
(\mathrm{J} / \mathrm{g})\end{array}$ \\
\hline 1 & -13 & & & & & \\
2 & -15 & & & & & \\
3 & -11 & 81 & 18.4 & 155 & 21.8 & 3.4 \\
4 & -20 & & & & & \\
5 & -20 & & & & & \\
6 & -13 & & & & & 30.0 \\
7 & -8 & 43 & 16.7 & 156 & 46.7 & 0 \\
8 & -11 & 56 & 22.5 & 142 & 22.5 & 83.0 \\
9 & 0 & & & 146,158 & 83.0 & \\
\hline
\end{tabular}

a blank space indicates that no transition was observed

corrected $\Delta H_{\mathrm{m}}$ value subtracts $\Delta H_{\mathrm{c}}$ value from $\Delta H_{\mathrm{m}}$ determined from software: corrected $\Delta H_{\mathrm{m}}=\Delta H_{\mathrm{m}}-\Delta H_{\mathrm{c}}$ 


\section{Literature Cited}

1. Pfeifer, B. A.; Admiraal, S. J.; Gramajo, H.; Cane, D. E.; Khosla, C., Biosynthesis of complex polyketides in a metabolically engineered strain of E. coli. Science 2001, 291 (5509), 1790-1792.

2. Killmann, H.; Videnov, G.; Jung, G.; Schwarz, H.; Braun, V., Identification of receptor binding sites by competitive peptide mapping: phages T1, T5, and phi 80 and colicin M bind to the gating loop of FhuA. J Bacteriol 1995, 177 (3), 694-698.

3. Datsenko, K. A.; Wanner, B. L., One-step inactivation of chromosomal genes in Escherichia coli K-12 using PCR products. Proc Natl Acad Sci U S A 2000, 97 (12), 6640-6645.

4. Gibson, D. G.; Young, L.; Chuang, R.-Y.; Venter, J. C.; Hutchison III, C. A.; Smith, H., Enzymatic assembly of DNA molecules up to several hundred kilobases. Nat Methods 2009, 6 (5), 343-345.

5. Thuronyi, B. W.; Privalsky, T. M.; Chang, M. C., Engineered fluorine metabolism and fluoropolymer production in living cells. Angew Chem Int Ed Engl 2017, 56 (44), 13637-13640.

6. Blaisse, M. R.; Dong, H.; Fu, B.; Chang, M. C., Discovery and engineering of pathways for production of $\alpha$-branched organic Acids. J Am Chem Soc 2017, 139 (41), 14526-14532.

7. Bond-Watts, B. B.; Bellerose, R. J.; Chang, M. C., Enzyme mechanism as a kinetic control element for designing synthetic biofuel pathways. Nat Chem Biol 2011, 7 (4), 222-227.

8. Stein, R. L., Kinetics of enzyme action: essential principles for drug hunters. John Wiley \& Sons: 2011.

9. Hill, A. V., The possible effects of the aggregation of the molecules of haemoglobin on its dissociation curves. J Physiol 1910, 40, 4-7.

10. Ushimaru, K.; Sangiambut, S.; Thomson, N.; Sivaniah, E.; Tsuge, T., New insights into activation and substrate recognition of polyhydroxyalkanoate synthase from Ralstonia eutropha. Appl Microbiol Biotechnol 2013, 97 (3), 1175-1182.

11. Jia, K.; Cao, R.; Hua, D. H.; Li, P., Study of class I and class III polyhydroxyalkanoate (PHA) synthases with substrates containing a modified side chain. Biomacromolecules 2016, 17 (4), $1477-1485$.

12. Wodzinska, J.; Snell, K.; Rhomberg, A.; Sinskey, A.; Biemann, K.; Stubbe, J., Polyhydroxybutyrate synthase: evidence for covalent catalysis. J Am Chem Soc 1996, 118 (26), 6319-6320.

13. Akdoğan, M.; Çelik, E., Purification and characterization of polyhydroxyalkanoate (PHA) from a Bacillus megaterium strain using various dehydration techniques. J Chem Technol Biotechnol 2018, 93 (8), 2292-2298.

14. Blaisse, M. R.; Fu, B.; Chang, M. C., Structural and biochemical studies of substrate selectivity in Ascaris suum thiolases. Biochemistry 2018, 57 (22), 3155-3166.

15. Inoue, Y.; Sano, F.; Nakamura, K.; Yoshie, N.; Saito, Y.; Satoh, H.; Mino, T.; Matsuo, T.; Doi, Y., Microstructure of copoly (3-hydroxyalkanoates) produced in the anaerobic-aerobic activated sludge process. Polym Int 1996, 39 (3), 183-189.

16. Dai, Y.; Lambert, L.; Yuan, Z.; Keller, J., Characterisation of polyhydroxyalkanoate copolymers with controllable four-monomer composition. J Biotechnol 2008, 134 (1-2), 137-145.

17. Yuan, W.; Jia, Y.; Tian, J.; Snell, K. D.; Müh, U.; Sinskey, A. J.; Lambalot, R. H.; Walsh, C. T.; Stubbe, J., Class I and III polyhydroxyalkanoate synthases from Ralstonia eutropha and Allochromatium vinosum: characterization and substrate specificity studies. Arch Biochem Biophys 2001, 394 (1), 87-98. 
18. Pearce, R. P.; Marchessault, R. H., Melting and crystallization in bacterial poly (betahydroxyvalerate), PHV, and blends with poly (beta-hydroxybutyrate-co-hydroxyvalerate). Macromolecules 1994, 27 (14), 3869-3874.

19. Domínguez-Díaz, M.; Meneses-Acosta, A.; Romo-Uribe, A.; Peña, C.; Segura, D.; Espin, G., Thermo-mechanical properties, microstructure and biocompatibility in poly- $\beta$-hydroxybutyrates (PHB) produced by OP and OPN strains of Azotobacter vinelandii. Eur Polym J 2015, 63, 101112.

20. Scandola, M.; Ceccorulli, G.; Doi, Y., Viscoelastic relaxations and thermal properties of bacterial poly (3-hydroxybutyrate-co-3-hydroxyvalerate) and poly (3-hydroxybutyrate-co-4hydroxybutyrate). Int J Biol Macromol 1990, 12 (2), 112-117.

21. Kunioka, M.; Tamaki, A.; Doi, Y., Crystalline and thermal properties of bacterial copolyesters: poly (3-hydroxybutyrate-co-3-hydroxyvalerate) and poly (3-hydroxybutyrate-co-4hydroxybutyrate). Macromolecules 1989, 22 (2), 694-697.

22. Asrar, J.; Valentin, H. E.; Berger, P. A.; Tran, M.; Padgette, S. R.; Garbow, J. R., Biosynthesis and properties of poly (3-hydroxybutyrate-co-3-hydroxyhexanoate) polymers. Biomacromolecules 2002, 3 (5), 1006-1012.

23. Watanabe, Y.; Ishizuka, K.; Furutate, S.; Abe, H.; Tsuge, T., Biosynthesis and characterization of novel poly (3-hydroxybutyrate-co-3-hydroxy-2-methylbutyrate): thermal behavior associated with $\alpha$-carbon methylation. $R S C A d v$ 2015, 5 (72), 58679-58685. 